\title{
Double trace interfaces
}

\author{
Charles M. Melby-Thompson ${ }^{a}$ and Cornelius Schmidt-Colinet ${ }^{b}$ \\ ${ }^{a}$ Department of Physics, Fudan University, \\ 220 Handan Road, 200433 Shanghai, China \\ ${ }^{b}$ Arnold Sommerfeld Center, Ludwig-Maximilians-Universität, \\ Theresienstraße 37, 80333 München, Germany \\ E-mail: charlesmelby@gmail.com, cornelius.sc@gmail.com
}

ABSTRACT: We introduce and study renormalization group interfaces between two holographic conformal theories which are related by deformation by a scalar double trace operator. At leading order in the $1 / N$ expansion, we derive expressions for the two point correlation functions of the scalar, as well as the spectrum of operators living on the interface. We also compute the interface contribution to the sphere partition function, which in two dimensions gives the boundary $g$ factor. Checks of our proposal include reproducing the $g$ factor and some defect overlap coefficients of Gaiotto's RG interfaces at large $N$, and the two-point correlation function whenever conformal perturbation theory is valid.

Keywords: AdS-CFT Correspondence, Conformal and W Symmetry, Renormalization Group

ARXIV EPrint: 1707.03418

LMU-ASC $72 / 17$ 


\section{Contents}

1 Introduction 1

2 Double trace interfaces 3

2.1 Double trace interfaces as a mixed boundary value problem 5

2.2 Holographic renormalization and correlation functions 6

2.3 Interface fusion and other generalizations 9

3 Green's function from harmonic methods $\quad 9$

$\begin{array}{ll}3.1 & \text { Wave equation on } H^{d+1} \\ 3.2 & 10\end{array}$

$\begin{array}{ll}3.2 \text { Green's function } & 11\end{array}$

$\begin{array}{lll}3.3 & \text { Green's function and the bulk-boundary propagator } & 12\end{array}$

$\begin{array}{lll}3.4 & \text { Interface operator spectrum } & 15\end{array}$

4 Correlation functions $\quad 16$

$\begin{array}{lll}4.1 & \text { Evaluation of the two-point functions } & 16\end{array}$

$\begin{array}{lll}4.1 .1 & \left\langle\varphi_{+}(x) \varphi_{+}\left(x^{\prime}\right)\right\rangle \text { and }\left\langle\varphi_{-}(x) \varphi_{-}\left(x^{\prime}\right)\right\rangle & 16\end{array}$

$\begin{array}{lll}4.1 .2 & \left\langle\varphi_{-}(\chi) \varphi_{+}\left(\chi^{\prime}\right)\right\rangle & 17\end{array}$

$\begin{array}{lll}4.2 & \text { Fusion channels and defect spectrum } & 18\end{array}$

$\begin{array}{ll}\text { 4.2.1 Bulk fusion channel } & 19\end{array}$

$\begin{array}{ll}4.2 .2 \text { Boundary fusion channel } & 21\end{array}$

5 Interface partition function $\quad \mathbf{2 2}$

5.1 Regulator 23

5.2 Sphere free energy and the $g$-factor 24

6 Comparison to field theory results $\quad 26$

6.1 Coefficients from conformal perturbation theory 26

6.2 Checks from minimal model holography in $d=2 \quad 32$

6.2.1 RG interface construction at finite $N$ and $k \quad 33$

6.2.2 The RG interface in the 't Hooft limit 34

$\begin{array}{lll}6.2 .3 & \text { RG interface boundary entropy } & 36\end{array}$

$\begin{array}{ll}\text { 6.2.4 Matching of coefficients for two-point functions } & 37\end{array}$

$\begin{array}{lll}7 & \text { Conclusions and discussion } & 40\end{array}$

A Coordinate systems on hyperbolic space $\quad 41$

A.1 Standard coordinates 41

$\begin{array}{lll}\text { A.2 Janus coordinates } & 41\end{array}$

A.3 Coordinates on $H^{d} \quad 42$

B Bulk-boundary propagator from dual integral equations 43 
$\begin{array}{ll}\text { E Hypergeometric functions } & 47\end{array}$

$\begin{array}{lll}\text { E.1 Identities } & 48\end{array}$

$\begin{array}{llr}\text { E.1.1 Euler identities } & 48\end{array}$

E.1.2 Kummer's connection formulas 48

E.1.3 Sum relations 48

E.2 Integrals 49

E.3 Index integrals of hypergeometric functions 49

E.4 Three-term relations for ${ }_{3} F_{2}(1) \quad 49$

$\begin{array}{ll}\text { F Conventions for } \mathfrak{s u}(N) & 50\end{array}$

\section{Introduction}

Conformal defects have played an important role in the development of conformal field theory (CFT). Of particular interest for many purposes are conformal interfaces, those interfaces separating two different CFTs that preserve a maximal subgroup of the conformal group.

A particularly interesting class of interfaces is given by renormalization group (RG) interfaces [1], which are associated to a renormalization group flow from $\mathrm{CFT}_{1}$ to $\mathrm{CFT}_{2}$. In addition to being of intrinsic interest, such defects may provide new tools to study the behavior of renormalization group flows. Various such interfaces, both approximate [2-4] and (in the presence of supersymmetry) exact $[1,5,6]$ have been constructed, but in general it is difficult to compute observables that are not protected by symmetry. In particular, we are not aware of computations of two-point correlation functions in the particular case of RG interfaces.

Within the AdS/CFT correspondence, conformal interfaces are typically realized using the Janus construction [7], ${ }^{1}$ for which various approximate and (in the supersymmetric case) exact solutions are known (see e.g. [8-11]). The construction takes advantage of the $\mathrm{SO}(d, 1)$ symmetry preserved by the interface to slice the bulk geometry by copies of hyperbolic space, ${ }^{2}$

$$
d s^{2}=d \beta^{2}+f(\beta) d s_{H^{d}}^{2} .
$$

Pure hyperbolic space corresponds to $f(\beta)=\cosh ^{2} \beta$. The deformation of $f(y)$ away from this is sourced by scalar field gradients $\phi(\beta)$, the details of which depend on the scalar

\footnotetext{
${ }^{1}$ Bulk D-branes can also play the role of interfaces. This can be understood as the thin wall limit of the Janus construction.

${ }^{2}$ In this paper we work exclusively in Euclidean signature, in which case the vacuum bulk geometry is $(d+1)$-dimensional hyperbolic space $H^{d+1}$, and the conformal group is $\mathrm{SO}(d+1,1)$.
} 


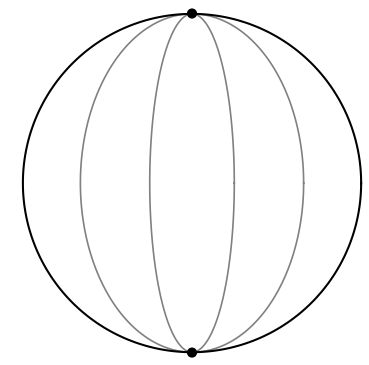

(a)

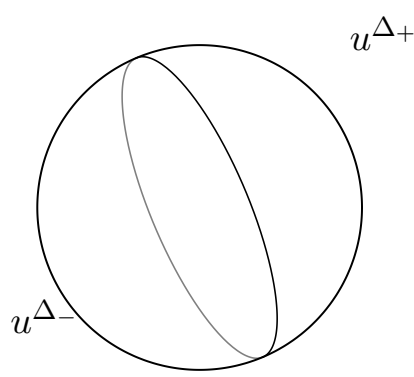

(b)

Figure 1. (a) Janus coordinates: $H^{d+1}$ is sliced by copies of $H^{d}$, which intersect along the defect. (b) Mixed boundary conditions on the two halves of the boundary of $H^{d+1}$.

potential. The bulk equations of motion are in general difficult to solve, and even in those cases where solutions are available, simple observables such as two-point correlation functions are difficult to compute: the only computation of a (non-protected) holographic two-point function we are aware of was performed in [12].

Holographic realizations of RG interfaces have appeared in the literature: see, for example, $[13,14]$. The purpose of this paper is to introduce and study a type of holographic RG interface that we refer to as (holographic) double trace interfaces. It has been known since the work of [15] that whenever the gravitational dual of a CFT has a scalar field whose mass lies in the unitarity window $-\frac{d^{2}}{4} \leq m^{2} \leq-\frac{d^{2}}{4}+1$, there are two consistent choices of boundary asymptotics. ${ }^{3}$ The two different choices lead to two different CFTs on the boundary, with different spectra. For one choice, the scalar field $\phi$ is dual to a gaugeinvariant (single trace) operator $\varphi_{+}$of dimension $\Delta_{+}$, while the other choice leads to an operator $\varphi_{-}$of dimension $\Delta_{-}$. These two CFTs are related by RG flow from CFT - to $\mathrm{CFT}_{+}$, which is initiated on the CFT side through perturbation by the "double trace" operator $\left(\varphi_{-}\right)^{2}$. This is implemented holographically by imposing on the scalar field boundary conditions of mixed Dirichlet-Neumann type; renormalization group flow from the UV to the IR is realized in terms of the dominant asymptotics in the near-boundary and deep bulk regions, respectively. These RG flows are particularly simple at large $N$. This can be traced to the fact that their effects are due entirely to the asymptotics of quantum fluctuations, and as a result, gravitational backreaction occurs only at loop level. As a result, the leading contribution to any computation takes place on a pure AdS background. This fact makes feasible, at least at leading order, computations that are impractical in the general case.

Consider hyperbolic space $H^{d+1}$, with its boundary divided into two regions, $A_{+}$and $A_{-}$(figure 1), where the local physics is described by CFT + and CFT - respectively. Near boundary region $A_{+}$, the quantum fluctuations of the bulk scalar field $\phi$ should have scaling dimension $\Delta_{+}$, while those near boundary region $A_{-}$should have dimension $\Delta_{-}$. The asymptotics of quantum fluctuations contribute to diagrammatic computations through the particular choice of bulk Green's function $G$ for $\phi$. The choice of Green's function is

\footnotetext{
${ }^{3}$ It is possible and interesting to relax this assumption, but doing so breaks unitarity. We restrict ourselves in this paper to unitary theories.
} 
therefore what determines all properties of the holographic interface, and sections 2 and 3 are devoted to its analysis.

With the Green's function in hand, in principle all observables associated to the interfaces can be computed by using this Green's function in all Witten diagrams. In this paper, we focus on the simplest observables that can be derived from $G$ : (1) the two-point correlation function at tree level, and (2) the one-loop partition function. From the correlation function one can further extract the spectrum of non-trivial defect operators. The two-point function can also be compared with CFT results. In particular, we show that our bulk expressions reproduce results that we derive in conformal perturbation theory. Furthermore, from the conformal block expansion we can read off relations between the bulk and bulk-boundary OPE coefficients, which allows us to derive an expression for the defect overlap coefficients for certain operators in the large $N$ limit. As an example, we reproduce the large $N$ behavior of overlap coefficients for the interfaces of [5] between adjacent $\mathcal{W}_{N}$ minimal models. We further compute the contribution of Gaiotto's interface to the sphere partition function in the large $N$ limit, and show that it exactly matches the interface contribution to the bulk one-loop partition function.

The logic of this paper is as follows. Section 2 gives a detailed definition of holographic double trace interfaces, and discusses methods available for deriving the bulk Green's functions for an arbitrary interface geometry. Section 3 turns to the explicit evaluation of the Green's function in the case of a spherical interface, which is done using the more powerful tools of harmonic analysis on $H^{d}$; these tools also allow us to derive the spectrum of interface operators. Section 4 treats the evaluation of the CFT two-point function at leading order in the $1 / N$ expansion, from which we extract the dimensions of a sequence of primary operators living on the interface, matching the results from section 3 . Section 5 computes the leading contribution of the interface to the partition function by evaluating the one-loop vacuum bubble diagram.

Section 6 is devoted to computations in CFT, which provide two tests of our results. As the first, we derive the CFT two-point function in the presence of double trace interfaces within conformal perturbation theory, and show that it matches our bulk computation in parameter regimes where both descriptions are valid. The second is to derive within the higher spin gravity/WCFT duality of [16] the boundary $g$-factor and several overlap coefficients for the RG interfaces of [5] joining the $\mathcal{W}_{N, k}$ and $\mathcal{W}_{N, k-1}$ minimal models. We find that both match the results of sections 4 and 5 . We close with a summary of our conclusions and a list of interesting questions and problems for the future.

\section{Double trace interfaces}

The construction of a double trace interface begins with a pair of $d$-dimensional unitary $\mathrm{CFT}_{\mathrm{s}}, \mathrm{CFT}_{ \pm}$, which have dual descriptions in terms of a single gravitational theory on a weakly curved AdS space, and are related by the choice of boundary condition for a bulk scalar field $\phi$ with $m^{2}=-\frac{d^{2}}{4}+\nu$. We take the mass to lie in the unitarity window, defined by $0<\nu<1$. The two CFTs therefore differ at leading order in the $1 / N$ expansion by the choice of dimension $\Delta_{ \pm}=\frac{d}{2} \pm \nu$ for a single operator $\varphi_{ \pm}$. 
Our goal is to describe a conformal interface separating a region $A_{+}$whose local physics is that of $\mathrm{CFT}_{+}$, and the complementary region $A_{-}$described by CFT. . How does one realize such an interface? The AdS/CFT dictionary says that the field $\phi$ should have boundary condition $\Delta_{+}$near the CFT + boundary, and boundary condition $\Delta_{-}$near the CFT_ region. To be more precise about what we mean, consider Poincaré patch coordinates $X=(u, \chi)$ with metric $^{4}$

$$
d s_{H^{d+1}}^{2}=\frac{d u^{2}+d s_{\mathbb{R}^{d}}^{2}(\chi)}{u^{2}} .
$$

By boundary condition, we mean that any configuration of the field $\phi$ appearing in the path integral must fall off near the boundary as

$$
\phi(u, \chi)= \begin{cases}\Psi_{+}(\chi) u^{\Delta_{+}}+O\left(u^{\Delta_{+}+2}\right), & \chi \in A_{+} \\ \Psi_{-}(\chi) u^{\Delta_{-}}+O\left(u^{\Delta_{-}+2}\right), & \chi \in A_{-} .\end{cases}
$$

This is accomplished in Witten diagrams by making a particular choice of inverse for the kinetic operator. The interface is therefore implemented in the bulk by choosing the appropriate bulk Green's function.

To be explicit, a double trace interface is obtained by imposing the following conditions on the Green's function $G$ :

(A) $G$ satisfies the defining equation ${ }^{5}$

$$
\left(-\square+m^{2}\right) G\left(X ; X^{\prime}\right)=\delta^{(d+1)}\left(X, X^{\prime}\right) .
$$

(B) As $X^{\prime}=\left(u^{\prime}, \chi^{\prime}\right)$ approaches a boundary point $\chi^{\prime} \in A_{ \pm}$,

$$
G\left(X ; X^{\prime}\right)= \pm \frac{1}{2 \nu} u^{\prime \Delta_{ \pm}} K^{ \pm}\left(X ; \chi^{\prime}\right)+O\left(u^{\prime \Delta_{ \pm}+2}\right) \quad \text { as } u^{\prime} \rightarrow 0 .
$$

The form of $K^{ \pm}$is not important for this definition, but it is in fact the bulk-boundary propagator associated to the region $A_{ \pm}$. The factor of $\pm \frac{1}{2 \nu}$ is the standard prefactor $\frac{1}{2 \Delta_{ \pm}-d}$.

We consider here two methods of solving these conditions. The first is harmonic analysis: when the bulk geometry can be expressed as a warped product of a symmetric space over an interval, the decomposition in terms of Laplacian eigenfunctions reduces the above equations to an ODE. This method works whenever the wave equation is separable in coordinates respecting the boundary geometry of the defect, as happens when the interface is spherical or planar. Otherwise, one must use the more general methods developed to deal with mixed boundary value problems for partial differential equations; for a thorough treatment of this subject, see for example [17]. As a simple example, we will outline at the end of this section the application of such methods to the derivation of the bulk-boundary propagator in the case of spherical defects; a full derivation using these methods is offered in appendix B.

\footnotetext{
${ }^{4}$ We work throughout in units such that the AdS length $\ell_{\text {AdS }}=1$.

${ }^{5}$ We use $\delta(x, y)$ to denote the covariant delta function, $\delta(x, y)=\frac{1}{\sqrt{g}} \delta(x-y)$.
} 


\subsection{Double trace interfaces as a mixed boundary value problem}

The Green's function solves a boundary value problem in which the boundary is split into two regions $A_{+}$and $A_{-}$, such that the function in question has Dirichlet-like boundary conditions on $A_{+}$, but Neumann-like boundary conditions on $A_{-}$. Such problems are known as mixed boundary value problems. (This is not to be confused with "mixed boundary conditions", otherwise known as Robin boundary conditions, which refer to a spatially homogeneous linear combination of Dirichlet and Neumann boundary conditions.)

We begin by writing the mixed Green's function in the form

$$
G\left(X ; X^{\prime}\right)=G_{\Delta_{-}}\left(X ; X^{\prime}\right)+\mathcal{H}\left(X ; X^{\prime}\right)
$$

where $G_{\Delta_{-}}$is the homogeneous Green's function for $\Delta_{-}$asymptotics. Then $\mathcal{H}$ satisfies the free scalar equation, so it can be written as the convolution of a function on the boundary of $H^{d+1}$ with $K_{\Delta_{-}}\left(X ; x^{\prime}\right)$, the bulk-boundary propagator for CFT_.

Let $K^{+}\left(u, \chi ; \chi^{\prime}\right)$ be the mixed bulk-boundary propagator associated to a boundary point $\chi^{\prime} \in A_{+}$. This function is determined by the following properties:

$[\mathrm{K} 1]\left(-\square+m^{2}\right) K^{+}\left(u, \chi ; \chi^{\prime}\right)=0$,

$[\mathrm{K} 2]\left[K^{+}\right]_{\Delta_{-}}\left(\chi ; \chi^{\prime}\right)=\delta\left(\chi, \chi^{\prime}\right)$ for $\chi \in A_{+}$,

$[\mathrm{K} 3]\left[K^{+}\right]_{\Delta_{+}}\left(\chi ; \chi^{\prime}\right)=0$ for $\chi \in A_{-}$.

Here, by $[f]_{\Delta}$ we mean the coefficient of $u^{\Delta}$ in the expansion of $f$ as $u \rightarrow 0 . K^{+}$is given in terms of the Green's function by the standard relation

$$
K^{+}\left(u, \chi ; \chi^{\prime}\right)=\lim _{u^{\prime} \rightarrow 0} \frac{2 \nu}{u^{\prime \Delta_{+}}} G\left(u, \chi ; u^{\prime}, \chi^{\prime}\right), \quad \chi^{\prime} \in A_{+} .
$$

We claim that

$$
\mathcal{H}\left(X ; X^{\prime}\right)=\frac{1}{2 \nu} \int_{A_{+}} d^{d} \chi^{\prime \prime} K^{+}\left(X ; \chi^{\prime \prime}\right) K_{\Delta_{-}}\left(X^{\prime} ; \chi^{\prime \prime}\right)
$$

where $K_{\Delta_{-}}$is the bulk-boundary propagator for the $\Delta_{-}$CFT. Recalling that

$$
\left[G_{\Delta_{ \pm}}\right]_{\Delta_{ \pm}}= \pm \frac{1}{2 \nu} K_{\Delta_{ \pm}}
$$

it is straightforward to verify that as a function of $(u, \chi), H$ satisfies the asymptotic conditions

$$
\begin{array}{ll}
{[\mathcal{H}]_{\Delta_{-}}\left(\chi ; u^{\prime}, \chi^{\prime}\right)=-\left[G_{\Delta_{-}}\right]_{\Delta_{-}}} & \chi \in A_{+} \\
{[\mathcal{H}]_{\Delta_{+}}\left(\chi ; u^{\prime}, \chi^{\prime}\right)=0} & \chi \in A_{-} .
\end{array}
$$

Defining $G$ as in (2.5) implies that it satisfies both conditions (A) and (B). Equations (2.5) and (2.7) therefore express $G$ in terms of $K^{+}$, reducing the problem to solving [K1]-[K3].

We see thus that observables of double trace interfaces can be expressed in terms of the bulk-boundary propagator, and thus it is this object that will be the primary focus of what follows. We focus in particular on the case of a spherical defect. This case is 
special because it preserves a maximal subgroup of the conformal group, allowing us to solve the problem as an ODE using harmonic analysis on $H^{d}$. This is done in section 3. For any other shape, it is necessary to solve for $K$ as a mixed boundary value problem. To illustrate this process, we show in detail how this can be done for the spherical interface in appendix B.

The remainder of the section will be occupied with holographic renormalization and the extraction of correlation functions in section 2.2 , and some comments on the case of general interface shapes in section 2.3 .

\subsection{Holographic renormalization and correlation functions}

Let us now consider the question of how to extract correlation functions from the bulkboundary propagator associated to a general double trace interface. The AdS/CFT dictionary states that for each bulk field $\phi$ dual to a scalar operator $\varphi$, the solution to the equations of motion can be expanded in the form

$$
\phi(u, \chi)=a_{J} J(\chi) u^{\Delta_{J}}+\psi(\chi) u^{\Delta_{\varphi}}+\cdots
$$

where $J$ denotes a source, $\psi(\chi)$ is proportional to the one-point function $\langle\varphi(\chi)\rangle_{J}$ in the presence of $J$, and all other terms are local functionals of $J$ and $\varphi ; a_{J}$ is a free parameter that we will fix later. Note $\Delta_{J}+\Delta_{\varphi}=d . \quad J$ and $\psi$ are locally independent, but are determined by each other upon requiring non-singular behavior in the bulk. Correlation functions are obtained by the statement that the gravitational partition function with boundary conditions $J$ is equal to the generating functional of the CFT with source $J$ :

$$
Z_{\mathrm{CFT}}(J)=Z_{\text {gravity }}\left(\phi \sim J u^{\Delta_{J}}+\cdots\right) .
$$

Defining $W=\log Z$, the connected correlation functions are

$$
\langle\varphi(\chi)\rangle_{J}=\left.a_{\varphi} \psi(\chi)\right|_{J}=\frac{\delta W(J)}{\delta J(\chi)} \quad\left\langle\varphi(\chi) \varphi\left(\chi^{\prime}\right)\right\rangle_{J}=\frac{\delta W(J)}{\delta J(\chi) \delta J\left(\chi^{\prime}\right)},
$$

where the value of $a_{\varphi}$ is determined by the effective action. Note that, due to the presence of $a_{J}$ in (2.11), this equation differs from the standard one by a factor of $a_{J}$. This is a matter of the normalization of the operator dual to $J$. It would be most natural to choose $a_{J}$ such that $a_{\varphi}=1$. The standard normalization, however, sets $a_{J}=1$. If $\phi$ has the $\Delta_{-}$quantization, $a_{\varphi}$ is negative, which flips the sign of certain correlators relative to the natural expectation in CFT. Because the $a_{\varphi}=1$ normalization is ubiquitous in the literature, we choose $a_{J}=1$ for the $\Delta_{+}$quantization; to obtain the natural sign for the mixed two-point functions, we therefore choose $a_{J}=-1$ for the $\Delta_{-}$quantization. We will see in section 4 that this convention reproduces the sign of $\left\langle\varphi_{+} \varphi_{-}\right\rangle$that is natural in conformal perturbation theory.

In the semi-classical limit $W$ is expressed in terms of the on-shell classical gravitational action $S_{\mathrm{os}}, W=-S_{\mathrm{os}}$, so this is the quantity we deal with for the rest of the section. To render the variations well-defined, one requires a well-behaved variational principle. In particular, this implies that if $\phi=\phi_{c}+\delta \phi$, where $\phi_{c}$ solves the bulk equations and $\delta \phi$ 
has $u^{\Delta_{\varphi}}$ asymptotics, then the variation of the action must be finite. As is well known, to accomplish this requires the inclusion of local counterterms (holographic renormalization), and the counterterms we add determine the allowed fluctuations.

Since our system involves both boundary conditions for $\phi$, let us first briefly review how this works when there is no interface. We restrict to $0<\nu<1$ as before, and expand near $u=0$ in the form

$$
\phi(u, \chi)=\phi_{-}(\chi) u^{\Delta_{-}}+\phi_{+}(\chi) u^{\Delta_{+}}+\cdots
$$

where $(\cdots)$ is irrelevant to what follows. Start with the variation of the bare on-shell action. Introduce a cutoff surface $u=\epsilon$, and let $S_{\epsilon}(\phi)$ be the cut-off bulk action. As usual, for $\phi$ on-shell we write

$$
\delta S_{\epsilon}(\phi)=\int d^{d+1} X \sqrt{g}\left(\nabla \phi \cdot \nabla \delta \phi+m^{2} \phi \delta \phi\right)=\int_{u=\epsilon} d^{d} \chi \sqrt{\gamma} \delta \phi \partial_{\hat{n}} \phi,
$$

where $\gamma$ is the induced metric on the cutoff surface, $\hat{n}$ is the outward-pointing unit normal, and we have dropped the term proportional to the equations of motion. Expanding in $\epsilon$ (' $\simeq$ ' means up to terms that vanish as $\epsilon \rightarrow 0$ ), we find

$$
\delta S_{\epsilon}(\phi) \simeq-\int d^{d} \chi\left(\Delta_{-} \phi_{-} \delta \phi_{-} \epsilon^{-2 \nu}+\Delta_{+} \phi_{+} \delta \phi_{-}+\Delta_{-} \phi_{-} \delta \phi_{+}\right) .
$$

We now add counterterms, which must render the variation finite. Furthermore, if we want $\Delta_{+}$boundary conditions, then the variation of the action should depend only on $\delta \phi_{-}$, while for $\Delta_{-}$boundary conditions, it should depend solely on $\delta \phi_{+}$. The first can be accomplished by the counterterm

$$
S_{\mathrm{ct}}^{\Delta_{+}}(\phi)=\frac{\Delta_{-}}{2} \int_{u=\epsilon} d^{d} \chi \sqrt{\gamma} \phi^{2} \quad \Longrightarrow \quad \delta S_{\mathrm{ct}}^{\Delta_{+}}(\phi) \simeq \int d^{d} \chi\left(\Delta_{-} \phi_{-} \delta \phi_{-} \epsilon^{-2 \nu}+2 \Delta_{-} \delta\left(\phi_{+} \phi_{-}\right)\right)
$$

which leads to

$$
\delta S^{\Delta_{+}}=\lim _{\epsilon \rightarrow 0}\left(\delta S_{\epsilon}+\delta S_{\mathrm{ct}}^{\Delta_{+}}\right)=\int d^{d} \chi(-2 \nu) \phi_{+} \delta \phi_{-} .
$$

Note that this gives $a_{\varphi}=-2 \nu$. We can obtain $\Delta_{-}$boundary conditions by instead using the counterterm

$$
S_{\mathrm{ct}}^{\Delta_{-}}=\frac{1}{2 \Delta_{-}} \int_{u=\epsilon} d^{d} \chi \sqrt{\gamma}\left(\partial_{\hat{n}} \phi\right)^{2} \Longrightarrow \delta S_{\mathrm{ct}}^{\Delta_{-}} \simeq \int d^{d} \chi\left(\Delta_{-} \phi_{-} \delta \phi_{-} \epsilon^{-2 \nu}+\Delta_{+} \delta\left(\phi_{+} \phi_{-}\right)\right)
$$

which gives

$$
\delta S^{\Delta_{-}}=\lim _{\epsilon \rightarrow 0}\left(\delta S_{\epsilon}+\delta S_{\mathrm{ct}}^{\Delta_{-}}\right)=\int d^{d} \chi(2 \nu) \phi_{-} \delta \phi_{+} .
$$

The bulk values of $\phi$ are determined by either one of $\phi_{+}$or $\phi_{-}$in terms of the bulkboundary propagator:

$$
\phi(u, \chi)=\int d^{d} \chi^{\prime} K_{\Delta_{+}}\left(u, \chi ; \chi^{\prime}\right) \phi_{-}\left(\chi^{\prime}\right)=-\int d^{d} \chi^{\prime} K_{\Delta_{-}}\left(u, \chi ; \chi^{\prime}\right) \phi_{+}\left(\chi^{\prime}\right)
$$


(the minus sign is due to $a_{J_{-}}=-1$ ). In $\mathrm{CFT}_{+}$, the source is $J_{+}=\phi_{-}$, and $\phi_{+}$is given by the relation

$$
\phi_{+}=\int d^{d} \chi^{\prime}\left[K_{\Delta_{+}}\right]_{\Delta_{+}}\left(\chi ; \chi^{\prime}\right) \phi_{-}\left(\chi^{\prime}\right) .
$$

From this we may obtain the standard result for the CFT + two-point function:

$$
\left\langle\varphi_{+}(\chi) \varphi_{+}\left(\chi^{\prime}\right)\right\rangle_{\mathrm{CFT}_{+}}=\frac{\delta^{2}\left(-S_{\mathrm{OS}}\right)}{\delta \phi_{-}(\chi) \delta \phi_{-}\left(\chi^{\prime}\right)}=2 \nu\left[K_{\Delta_{+}}\right]_{\Delta_{+}}\left(\chi ; \chi^{\prime}\right) .
$$

The same applied to CFT $-\left(\right.$ with $J_{-}=-\phi_{+}$) gives the usual value

$$
\left\langle\varphi_{-}(\chi) \varphi_{-}\left(\chi^{\prime}\right)\right\rangle_{\mathrm{CFT}_{-}}=\frac{\delta^{2}\left(-S_{\mathrm{os}}\right)}{\delta \phi_{+}(\chi) \delta \phi_{+}\left(\chi^{\prime}\right)}=-2 \nu\left[K_{\Delta_{-}}\right]_{\Delta_{-}}\left(\chi ; \chi^{\prime}\right) .
$$

Let us now turn to our case of interest, where the boundary is divided into a region $A_{+}$of $\mathrm{CFT}_{+}$and a region $A_{-}$of $\mathrm{CFT}_{-}$. We can still expand any on-shell field configuration $\phi$ as in equation (2.14). Our counterterms, and thus our identification of sources, is however different. We must use the counterterm $S_{\mathrm{ct}}^{\Delta_{+}}$in $A_{+}$, and $S_{\mathrm{ct}}^{\Delta_{-}}$in $A_{-}$. Using this counterterm, the variation of the on-shell action becomes:

$$
\delta S_{\mathrm{os}}=\int_{A_{+}} d^{d} \chi(-2 \nu) \phi_{+} \delta \phi_{-}+\int_{A_{-}} d^{d} \chi(+2 \nu) \phi_{-} \delta \phi_{+} .
$$

The source in $A_{+}$is $J_{+}=\left.\phi_{-}\right|_{A_{+}}$, while in $A_{-}$the source is $J_{-}=-\left.\phi_{+}\right|_{A_{-}}$. As before, $\phi_{+}$ and $\phi_{-}$are determined everywhere by these sources:

$$
\phi_{+}\left(\chi \in A_{+}\right)=\int_{A_{+}} d^{d} \chi^{\prime}\left[K^{+}\right]_{\Delta_{+}}\left(\chi ; \chi^{\prime}\right) J_{+}\left(\chi^{\prime}\right)-\int_{A_{-}} d^{d} \chi^{\prime}\left[K^{-}\right]_{\Delta_{+}}\left(\chi ; \chi^{\prime}\right) J_{-}\left(\chi^{\prime}\right),
$$

and similarly for $\phi_{-}$. Here we see $a_{J_{-}}=-1$ appearing again in the second term.

Let us use this to find the two-point function $\mathcal{G}_{++}\left(\chi, \chi^{\prime}\right)$ for $\chi, \chi^{\prime} \in A_{+}$. Assume we only have a source in $A_{+}$, so that $J_{-}=0$. The expression for the variation of the on-shell action tells us that now

$$
\delta\left(-S_{\mathrm{os}}\right)=\int_{A_{+}} d^{d} \chi(-2 \nu) \phi_{+} \delta J_{+},
$$

giving

$$
\left\langle\varphi_{+}(\chi)\right\rangle_{J_{+}}=\frac{\delta\left(-S_{\mathrm{os}}\right)}{\delta J_{+}(\chi)}=2 \nu \phi_{+}(\chi)
$$

and hence

$$
\mathcal{G}_{++}\left(\chi, \chi^{\prime}\right)=\left\langle\varphi(\chi) \varphi\left(\chi^{\prime}\right)\right\rangle=2 \nu \frac{\delta \phi_{+}\left(\chi^{\prime}\right)}{\delta J_{+}(\chi)}=2 \nu\left[K^{+}\right]_{\Delta_{+}}\left(\chi ; \chi^{\prime}\right) .
$$

Replication of this procedure yields the three independent two-point functions: for $\chi_{ \pm}, \chi_{ \pm}^{\prime} \in$ $A_{ \pm}$

$$
\begin{aligned}
& \mathcal{G}_{++}\left(\chi_{+}, \chi_{+}^{\prime}\right)=+2 \nu\left[K^{+}\right]_{\Delta_{+}}\left(\chi_{+} ; \chi_{+}^{\prime}\right) \\
& \mathcal{G}_{--}\left(\chi_{-}, \chi_{-}^{\prime}\right)=-2 \nu\left[K^{-}\right]_{\Delta_{-}}\left(\chi_{-} ; \chi_{-}^{\prime}\right) \\
& \mathcal{G}_{+-}\left(\chi_{+}, \chi_{-}^{\prime}\right)=-2 \nu\left[K^{-}\right]_{\Delta_{+}}\left(\chi_{+} ; \chi_{-}^{\prime}\right)=+2 \nu\left[K^{+}\right]_{\Delta_{-}}\left(\chi_{-}^{\prime} ; \chi_{+}\right)
\end{aligned}
$$

Note that (2.30) is invariant under $\left(+\leftrightarrow-, \nu \rightarrow-\nu, \chi \leftrightarrow \chi^{\prime}\right)$, as it should be. 


\subsection{Interface fusion and other generalizations}

As we have emphasized, the mixed boundary value problem approach can deal with more general geometries than the Janus approach. Let us take a moment to touch on a geometry relevant to a topic of particular interest for the theory of conformal interfaces: interface fusion.

The methods discussed above can be used to understand the fusion properties of two double trace interfaces with the opposite orientation. As a simple example, consider the case of two concentric spherical interfaces with opposite orientations, corresponding to CFT - on region $A_{-}$, which is interrupted by an annular region $A_{+}=\left\{x\left|R_{1}<\right| x \mid<R_{2}\right\}$ of $\mathrm{CFT}_{+}$. This configuration preserves $\mathrm{SO}(d)$ symmetry.

The Green's function is obtained using the tools outlined in this section (a detailed example is worked out in appendix B): expand the bulk-boundary propagator (or the Green's function) using spherical wave solutions of the bulk wave equation. The region where we impose condition [K2] is different from that of appendix $\mathrm{B}$, and so the ansatz relevant to the spherical interface - found in equation (B.6) - must be replaced by an ansatz appropriate to the new $A_{-}$. Similarly, the analog of (B.14), required to satisfy [K3], will now give a more complicated integral equation that must be solved to obtain $K$.

Carrying out this procedure explicitly is complicated, and we leave it for future work. Configurations with even smaller symmetry groups can in principle be considered, but the difficulty of solving the mixed boundary value problem increases quickly as the degree of symmetry is reduced.

\section{Green's function from harmonic methods}

Let us now turn to the explicit computation of the interface propagators in the case of a spherical interface. In this section we take the boundary to be spherical, and $A_{+}$to be a hemisphere. The computation is simplest in Janus coordinates on $H^{d+1}$ [7], which make the $\mathrm{SO}(d, 1)$ symmetry of the defect manifest. We will mostly use the coordinates

$$
d s_{H^{d+1}}=\frac{d z^{2}}{4 z^{2}(1-z)^{2}}+\frac{d s_{H^{d}}^{2}}{4 z(1-z)} \quad z \in(0,1)
$$

and reserve $x, x^{\prime}, \ldots$ to refer to points on the $H^{d}$ slice. (For a summary of the relationship of these to other useful coordinate systems on $H^{d+1}$, see appendix A.) In these coordinates, the boundary is split into two components: $A_{+}$, which lies at $z \rightarrow 1$, and $A_{-}$, at $z \rightarrow 0$. The interface lies at the boundary of $H^{d}$, with the limit taken along any surface of constant $z$.

To solve (A,B) we begin by decomposing $G$ with respect to eigenfunctions of the Laplacian on $H^{d}$. We choose a basis $\Psi_{s}(x)$ for the eigenfunctions,

$$
-\nabla_{H^{d}}^{2} \Psi_{s}(x)=\lambda_{s} \Psi_{s}(x),
$$


indexed by some parameters $s$. The index set is equipped with a measure $d \mu(s)$, with respect to which $\Psi_{s}(x)$ satisfies the normalization conditions:

$$
\begin{aligned}
\int d \mu(s) \Psi_{s}(x) \overline{\Psi_{s}\left(x^{\prime}\right)} & =\delta\left(x, x^{\prime}\right) \\
\int d^{d} x \sqrt{g_{H^{d}}} \Psi_{s}(x) \overline{\Psi_{s^{\prime}}(x)} & =\delta\left(s, s^{\prime}\right),
\end{aligned}
$$

with $\delta\left(s, s^{\prime}\right)$ the normalized delta function satisfying $\int d \mu(s) \delta\left(s, s^{\prime}\right) f(s)=f\left(s^{\prime}\right)$.

One explicit basis and its measure are given in detail in appendix C. This basis picks a point $p$ in $H^{d}$ and decomposes in spherical waves centered around this point. In this case, $s=(\sigma, \ell)$ where $\ell$ indexes the spherical harmonics on $S^{d-1}$, and $\sigma=\sigma_{s} \geq 0$ is defined by

$$
\lambda_{s}=\left(\frac{d-1}{2}\right)^{2}+\sigma_{s}^{2} .
$$

Since all the functions we use in this paper involve symmetric functions $F\left(x, x^{\prime}\right)$ of two variables on $H^{d}$, it is also useful to have a basis for these functions that are Laplacian eigenfunctions. As discussed in detail in appendix $\mathrm{C}$, this is straightforward in the spherical basis:

$$
J_{\sigma}\left(x, x^{\prime}\right)=\sum_{\ell} \Psi_{\sigma, \ell}(x) \overline{\Psi_{\sigma, \ell}\left(x^{\prime}\right)}
$$

is just such an eigenfunction. It depends only on the $\mathrm{SO}(d, 1)$-invariant cross-ratio $\xi$, which in Poincaré patch coordinates (A.9) on $H^{d}$ is $\frac{\left(x-x^{\prime}\right)^{2}}{4 y y^{\prime}}$. It further satisfies the useful identity

$$
\int_{0}^{\infty} d \sigma J_{\sigma}\left(x, x^{\prime}\right)=\delta\left(x, x^{\prime}\right) .
$$

A basis for the functions on $H^{d}$ in hand, our first task is to find the general solution to the wave equation on $H^{d+1}$ adapted to the Janus decomposition.

\subsection{Wave equation on $H^{d+1}$}

We use the metric (3.1). Performing separation of variables with respect to the Janus slicing, we look for solutions to the wave equation

$$
\left(-\nabla_{H^{d+1}}^{2}+m^{2}\right) \phi=0
$$

of the form $\phi(z, x)=\Phi(z) \Psi_{s}(x)$. This gives

$$
\left\{-[z(1-z)]^{d / 2+1} \frac{d}{d z} \frac{4}{[z(1-z)]^{d / 2-1}} \frac{d}{d z}+4 z(1-z) \lambda_{s}+m^{2}\right\} \Phi(z)=0 .
$$

The space of solutions is two-dimensional, but in what follows we will be interested in four different solutions:

$$
\begin{aligned}
& \Phi_{L}^{ \pm}(\sigma \mid z)=[4 z(1-z)]_{ \pm}^{\Delta_{ \pm} / 2}{ }_{2} F_{1}\left(\begin{array}{c|c}
\frac{1}{2} \pm \nu+i \sigma, \frac{1}{2} \pm \nu-i \sigma & z \\
1 \pm \nu &
\end{array}\right) \\
& \Phi_{R}^{ \pm}(\sigma \mid z)=[4 z(1-z)]^{\Delta_{ \pm} / 2}{ }_{2} F_{1}\left(\begin{array}{c|c}
\frac{1}{2} \pm \nu+i \sigma, \frac{1}{2} \pm \nu-i \sigma \\
1 \pm \nu
\end{array} \mid 1-z\right) .
\end{aligned}
$$


$\Phi_{L, R}^{ \pm}(\sigma \mid z)$ have the property that as we approach the left boundary $(z \rightarrow 0)$,

$$
\Phi_{L}^{ \pm}(\sigma \mid z) \sim z^{\Delta_{ \pm} / 2} \quad \text { as } \quad z \rightarrow 0,
$$

while as we approach the right boundary $(z \rightarrow 1)$,

$$
\Phi_{R}^{ \pm}(\sigma \mid z) \sim(1-z)^{\Delta_{ \pm} / 2} \quad \text { as } \quad z \rightarrow 1 .
$$

Therefore, $\Phi_{L}^{ \pm}$and $\Phi_{R}^{ \pm}$give bases with definite asymptotics $z^{\Delta_{ \pm} / 2}$ on left- and right-hand boundaries, respectively.

Having identified a basis of solutions, we can decompose any solution to the wave equation in the form

$$
f(z, x)=\sum_{a= \pm} \int d \mu(s) g_{L, R}^{a}(s) \Phi_{L, R}^{a}\left(\sigma_{s} \mid z\right) \Psi(s \mid x) .
$$

We are free to choose as we like whether to expand in terms of $\Phi_{L}^{ \pm}$or $\Phi_{R}^{ \pm}$. Note that when it will cause no confusion, we will frequently abbreviate $\Phi_{L}^{+}(\sigma \mid z)$ by $\Phi_{L}^{+}(z)$, and so forth.

Connection coefficients. In what follows, we will need the linear transformation between the bases $\Phi_{L}^{ \pm}$and $\Phi_{R}^{ \pm}$. This is given by Kummer's connection formulae (E.8a):

$$
\Phi_{L}^{a}=\sum_{b= \pm} A^{a b} \Phi_{R}^{b} \quad \Phi_{R}^{a}=\sum_{b= \pm} A^{a b} \Phi_{L}^{b}
$$

with

$$
A^{ \pm \pm}=\mp \frac{\cosh (\pi \sigma)}{\sin (\pi \nu)} \quad A^{ \pm \mp}=2^{ \pm 2 \nu} \frac{\Gamma(1 \pm \nu) \Gamma( \pm \nu)}{\Gamma\left(\frac{1}{2} \pm \nu+i \sigma\right) \Gamma\left(\frac{1}{2} \pm \nu-i \sigma\right)} .
$$

Note that the connection coefficients are symmetric under the exchange of $L \leftrightarrow R$. Applying the change of basis twice implies the consistency relation

$$
\begin{aligned}
& A^{ \pm \pm} A^{ \pm \pm}+A^{ \pm \mp} A^{\mp \pm}=1 \\
& A^{ \pm \pm} A^{ \pm \mp}+A^{ \pm \mp} A^{\mp \mp}=0 .
\end{aligned}
$$

\subsection{Green's function}

We are now in a position to decompose the Green's function with respect to the functions $\Psi_{s}$ and $\Phi_{R, L}^{ \pm}$. Actually, there are four linearly independent Green's functions $G^{a b}$ with $a, b= \pm$ :

$$
G^{a b}\left(X ; X^{\prime}\right) \sim\left\{\begin{array}{ll}
g_{1}\left(x, X^{\prime}\right) z^{\Delta_{a} / 2}+O\left(z^{\Delta_{a} / 2+1}\right) & z \rightarrow 0 \\
g_{2}\left(x, X^{\prime}\right)(1-z)^{\Delta_{b} / 2}+O\left([1-z]^{\Delta_{b} / 2+1}\right) & z \rightarrow 1
\end{array}\right\} .
$$

Thus the standard Green's function $G^{++}$has $\Delta_{+}$asymptotics on both boundary components, while that with $\Delta_{-}$asymptotics on the left boundary and $\Delta_{+}$asymptotics on the right boundary is $G^{-+}$.

Any Green's function satisfies the condition

$$
\left(-\nabla_{H^{d+1}}^{2}+m^{2}\right) G\left(X ; X^{\prime}\right)=\delta\left(X, X^{\prime}\right),
$$


where $\delta\left(X, X^{\prime}\right)$ is the covariant delta function on $H^{d+1}$. We begin with an ansatz for $G^{a b}$ in terms of eigenfunctions on $H^{d}$,

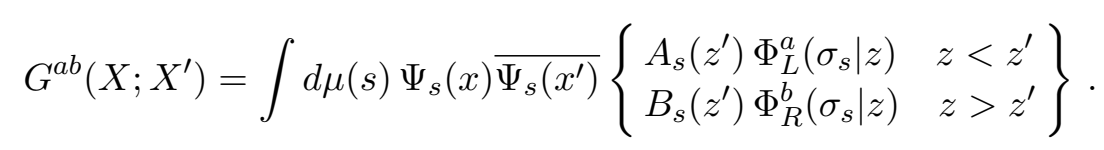

Applying $\left(-\nabla_{H^{d+1}}^{2}+m^{2}\right)$ and using the resolution (3.3) of the delta function on $H^{d},(3.20)$ becomes the condition

$$
A(z) \partial_{z} \Phi_{L}^{a}(z)-B(z) \partial_{z} \Phi_{R}^{b}(z)=2^{d-1}[z(1-z)]^{\frac{d}{2}-1}
$$

which is solved by

$$
A(z)=2^{d-1}[z(1-z)]^{\frac{d}{2}-1} \frac{\Phi_{R}^{a}(z)}{\mathcal{W}\left[\Phi_{R}^{b}, \Phi_{L}^{a}\right](z)}, \quad B(z)=2^{d-1}[z(1-z)]^{\frac{d}{2}-1} \frac{\Phi_{L}^{b}(z)}{\mathcal{W}\left[\Phi_{R}^{b}, \Phi_{L}^{a}\right](z)}
$$

with $\mathcal{W}[f, g](z)=f \partial_{z} g-g \partial_{z} f$ the Wronskian.

Define $W_{M N}^{a b}=\mathcal{W}\left[\Phi_{M}^{a}, \Phi_{N}^{b}\right]$, and $w_{M N}^{a b}$ by $W_{M N}^{a b}=w_{M N}^{a b} W_{R R}^{+-}$. The Wronskians are found from

$$
W_{R R}^{+-}=2^{d} \nu[z(1-z)]^{\frac{d}{2}-1}
$$

together with the values for the connection coefficients

$$
\begin{array}{lll}
w_{L R}^{+-}=A^{++} & w_{L R}^{-+}=-A^{--} & w_{L L}^{+-}=-1 \\
w_{L R}^{--}=A^{-+} & w_{L R}^{++}=-A^{+-}, &
\end{array}
$$

all others being determined by $w_{N M}^{b a}=-w_{M N}^{a b}$. This gives the final form for the Green's function:

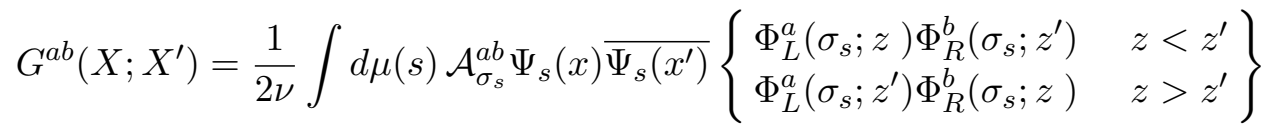

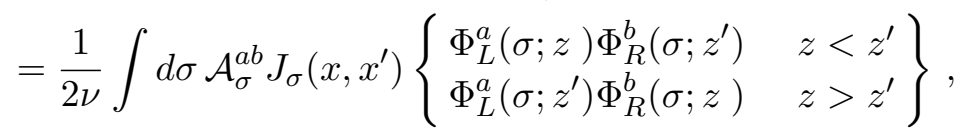

with $\mathcal{A}_{\sigma}^{a b}=\frac{1}{w_{R L}^{b a}(\sigma)}$ explicitly given by

$$
\begin{array}{ll}
\mathcal{A}_{\sigma}^{++}=A^{+-} & \mathcal{A}_{\sigma}^{+-}=A^{--} \\
\mathcal{A}_{\sigma}^{--}=-A^{-+} & \mathcal{A}_{\sigma}^{-+}=-A^{++}
\end{array}
$$

\subsection{Green's function and the bulk-boundary propagator}

Consider now the bulk-boundary propagator, which is obtained from the Green's function as follows: if $\rho$ is a defining function on $H^{d+1}$, then

$$
K\left(\rho, x ; x^{\prime}\right)=-\frac{1}{2 \Delta-d} \lim _{\rho^{\prime} \rightarrow 0} \frac{1}{\rho^{\prime \Delta}} G\left(\rho, x ; \rho^{\prime}, x^{\prime}\right)
$$


where $\Delta$ is the scaling dimension of the operator living at the boundary point $x^{\prime}$. In the coordinate system (3.1) (and in the conformal frame such that the boundary metric is $H^{d}$ ), the defining function is $\rho=\sqrt{4 z(1-z)}$.

Alternatively, the bulk-boundary propagator can be characterized by [K1]-[K3]. Denote by $K_{M}^{a b}\left(X ; x^{\prime}\right)(M=L, R)$ the bulk-boundary propagator for the $(a b)$ interface, with insertion at the point $x^{\prime}$ on boundary $M$. In the notation of section 2 this means, for example, that $K^{-}=K_{L}^{-+}$and $K^{+}=K_{R}^{-+}$. We give expressions for $K_{L}^{a b}$; the generalization to $K_{R}^{a b}$ is obvious. In the Janus conformal frame the conditions become

1. $\left(-\nabla_{H^{d+1}}^{2}+m^{2}\right) K_{L}^{a b}\left(X ; x^{\prime}\right)=0$.

2. The coefficient of $z^{\Delta_{-a} / 2}$ near the left-hand boundary $z \rightarrow 0$ is the covariant delta function $\delta\left(x, x^{\prime}\right)$.

3. The coefficient of $(1-z)^{\Delta_{-b} / 2}$ near the right-hand boundary $z \rightarrow 1$ vanishes.

The first and third properties imply that $K$ can be expanded in the form

$$
K\left(X ; x^{\prime}\right)=\int d \mu(s) \kappa_{s}\left(x^{\prime}\right) \Psi_{s}(x) \Phi_{R}^{b}\left(\sigma_{s} \mid z\right)
$$

To impose the second property, we use the connection relations $\Phi_{R}^{b}=A^{b+} \Phi_{L}^{+}+A^{b-} \Phi_{L}^{-}$, together with the fact that $\Phi_{L}^{ \pm}=\# z^{\Delta_{ \pm} / 2}+O\left(z^{\Delta_{ \pm} / 2+1}\right)$ as $z \rightarrow 0$. If we are to get the covariant delta function, the coefficient of this term must give the resolution of the delta function (3.3), implying

$$
\kappa_{s}\left(x^{\prime}\right)=\frac{1}{A^{b,-a}} \overline{\Psi_{s}\left(x^{\prime}\right)}
$$

Hence,

$$
K_{L}^{a b}\left(X ; x^{\prime}\right)=\int d \mu(s) \frac{1}{A^{b,-a}} \Psi_{s}(x) \overline{\Psi_{s}\left(x^{\prime}\right)} \Phi_{R}^{b}\left(\sigma_{s} \mid z\right) .
$$

A simple example is given by the standard bulk-boundary propagator with insertion on the left boundary, $K_{\Delta_{+}}=K_{L}^{++}$. In the spherical basis, $\int d \mu(s)=\int d \sigma \sum_{\ell}$. Carrying out the sum over $\ell$ gives

$$
\begin{aligned}
& K_{\Delta_{+}}\left(z, x ; x^{\prime}\right)=[4 z(1-z)]^{\Delta_{+} / 2} \times \\
& \quad \int_{0}^{\infty} d \sigma \frac{\nu}{4^{\nu}}\left|\frac{\Gamma\left(\frac{1}{2}+\nu+i \sigma\right)}{\Gamma(1+\nu)}\right|^{2} J_{\sigma}\left(x, x^{\prime}\right){ }_{2} F_{1}\left(\begin{array}{c}
\frac{1}{2}+\nu+i \sigma, \frac{1}{2}+\nu-i \sigma \\
1+\nu
\end{array} \mid 1-z\right) .
\end{aligned}
$$

This integral can be evaluated straightforwardly by expanding in a power series in $(1-z)$ and using the integral identity ${ }^{6}$

$$
\frac{1}{\pi} \int_{0}^{\infty} d s\left|\frac{\Gamma(a+i s) \Gamma(b+i s) \Gamma(c+i s)}{\Gamma(2 i s)}\right|_{2}^{2}{ }_{2} F_{1}\left(\begin{array}{c}
a+i s, a-i s \\
a+c
\end{array} \mid-x\right)=\frac{\Gamma(a+b) \Gamma(a+c) \Gamma(b+c)}{(1+x)^{a+b}} .
$$

\footnotetext{
${ }^{6}$ This is derived by applying the Olevskii transform to $(1+x)^{-a-b}$.
} 
Summing the series in $(1-z)$, we find

$$
K_{\Delta_{+}}\left(z, x ; x^{\prime}\right)=\frac{\Gamma\left(\Delta_{+}\right)}{\pi^{d / 2} \Gamma(\nu)}\left(\frac{2(z+\xi)}{\sqrt{z(1-z)}}\right)^{-\Delta_{+}}=\frac{\Gamma\left(\Delta_{+}\right)}{\pi^{d / 2} \Gamma(\nu)}\left(4 \Xi^{2}\right)^{-\Delta_{+}}
$$

with

$$
\Xi^{2}=\lim _{z^{\prime} \rightarrow 0} \sqrt{4 z^{\prime}\left(1-z^{\prime}\right)} \chi_{d+1}^{2}\left(X, X^{\prime}\right)=\frac{\xi\left(x, x^{\prime}\right)+z}{\sqrt{4 z(1-z)}},
$$

where $\chi_{d+1}^{2}$ is the cross ratio for $H^{d+1}$; see equation (C.13). Equation (3.37) is related, as it should be, by a Weyl transformation to the usual Poincaré patch expression. This can be seen by noting that $\Xi^{2}$ is a conformal covariant factor associated to our choice of defining functional, $\sqrt{4 z(1-z)}$. The corresponding object for the Poincaré patch is

$$
\Xi_{\text {p.p. }}^{2}\left(u, \vec{x} ; \vec{x}^{\prime}\right)=\lim _{u^{\prime} \rightarrow 0} u^{\prime} \frac{\left(\vec{x}-\vec{x}^{\prime}\right)^{2}+\left(u-u^{\prime}\right)^{2}}{4 u u^{\prime}}=\frac{1}{4} \frac{\left(\vec{x}-\vec{x}^{\prime}\right)^{2}+u^{2}}{u} .
$$

Replacing $\Xi$ by $\Xi_{\text {p.p. }}$ in (3.37) gives the standard bulk-boundary propagator.

Interface bulk-boundary propagator $\boldsymbol{K}_{\boldsymbol{L}}^{+-}$. The bulk-boundary propagator for a non-trivial defect is found in the same way. Equation (3.34) now takes the form

$$
K_{L}^{+-}\left(z, x ; x^{\prime}\right)=\frac{\sin \pi \nu}{\pi} \int_{0}^{\infty} d \sigma\left|\Gamma\left(\frac{1}{2}+i \sigma\right)\right|^{2} J_{\sigma}(\xi) \Phi_{R}^{-}(\sigma \mid z) .
$$

Using Euler's transformation we can write

$$
\Phi_{R}^{-}(\sigma \mid z)=z^{\Delta_{+} / 2}[4(1-z)]^{\Delta_{-} / 2}{ }_{2} F_{1}\left(\begin{array}{c}
\frac{1}{2}+i \sigma, \frac{1}{2}-i \sigma \\
1-\nu
\end{array} \mid 1-z\right) .
$$

Power expanding in $(1-z)$, the integral can be carried out using (3.36), and summing gives

$$
K_{L}^{+-}\left(z, x ; x^{\prime}\right)=\frac{\sin \pi \nu}{\pi} \frac{\Gamma(d / 2)}{\pi^{d / 2}} \frac{[4 z(1-z)]^{\Delta_{+} / 2}}{4^{\Delta_{+}}(1-z)^{\nu}}(1+\xi)^{-d / 2}{ }_{2} F_{1}\left(\begin{array}{c}
d / 2,1 \\
1-\nu
\end{array} \mid \frac{1-z}{1+\xi}\right) .
$$

Using Euler's transformation gives the form

$$
K_{L}^{+-}\left(z, x ; x^{\prime}\right)=\frac{\sin \pi \nu}{\pi} \frac{\Gamma(d / 2)}{\pi^{d / 2}}\left(4 \Xi^{2}\right)^{-\Delta_{+}}\left(\frac{\xi+z}{1-z}\right)^{\nu}{ }_{2} F_{1}\left(\begin{array}{c}
d / 2,-\nu \\
1-\nu
\end{array} \mid-\frac{1-z}{\xi+z}\right)
$$

which can also be nicely represented as

$$
K_{L}^{+-}=K_{L}^{++} \times \frac{\sin \pi \nu}{\pi} \frac{\Gamma(\nu) \Gamma(d / 2)}{\Gamma\left(\Delta_{+}\right)}\left(\frac{\xi+z}{1-z}\right)^{\nu}{ }_{2} F_{1}\left(\begin{array}{c}
d / 2,-\nu \\
1-\nu
\end{array} \mid-\frac{1-z}{\xi+z}\right) .
$$

Other bulk-boundary propagators. All other propagators can be obtained from these two using the relations

$$
K_{L}^{a, b}\left(z, x ; x^{\prime}\right)=K_{R}^{b, a}\left(1-z, x ; x^{\prime}\right) \quad K_{M}^{a, b}\left(z, x ; x^{\prime}\right)=\left.K_{M}^{-a,-b}\right|_{\nu \mapsto-\nu} .
$$




\subsection{Interface operator spectrum}

One of the key features to understand in any interface CFT is the spectrum of operators living on the defect. Fortunately, from the holographic point of view there is a simple and elegant way to identify the interface operators [18]. Say we have a single scalar field $\phi$ which couples only to the background geometry. If the background corresponds to a conformal interface, $\mathrm{SO}(d, 1)$ invariance implies the linearized equation of motion can be written in the form

$$
\left(-\nabla_{H^{d}}^{2}+\mathcal{D}\right) \phi(z, x)=0
$$

where $x$ is the coordinate on $H^{d}$, and $\mathcal{D}$ is a differential operator built using only the transverse coordinate $z$. If we expand $\phi$ in eigenmodes of the operator $\mathcal{D}$,

$$
\phi(z, x)=\sum_{a} \phi_{a}(x) \psi_{a}(z) \quad \mathcal{D} \psi_{a}(z)=m_{a}^{2} \psi_{a}(z),
$$

then $\phi_{a}(x)$ satisfies the standard scalar field equation on $H^{d}$ with mass $m_{a}^{2}$. Each $\phi_{a}$ is now the bulk dual to a defect operator of dimension

$$
\Delta_{a}=\frac{d-1}{2}+\nu_{a}, \quad \nu_{a}=\sqrt{\frac{(d-1)^{2}}{4}+m_{a}^{2}} .
$$

For double trace interfaces the analysis is particularly simple, as the equation of motion is simply the standard bulk equation of motion in Janus coordinates. The relevant eigenmodes can be found by making the substitution $i \sigma \rightarrow \nu_{a}$ in (3.10) and (3.11), giving us two convenient bases for the solution space,

$$
\begin{aligned}
& \psi_{a, L}^{ \pm}(z)=[4 z(1-z)]_{ \pm}^{\Delta_{ \pm} / 2}{ }_{2} F_{1}\left(\begin{array}{c}
\frac{1}{2} \pm \nu+\nu_{a}, \frac{1}{2} \pm \nu-\nu_{a} \\
1 \pm \nu
\end{array} \mid z\right) \\
& \psi_{a, R}^{ \pm}(z)=[4 z(1-z)]^{\Delta_{ \pm} / 2}{ }_{2} F_{1}\left(\begin{array}{c|c}
\frac{1}{2} \pm \nu+\nu_{a}, \frac{1}{2} \pm \nu-\nu_{a} & 1-z \\
1 \pm \nu
\end{array}\right.
\end{aligned}
$$

Our problem now is to identify the allowed values of $\nu_{a}$. Let us say that the left boundary has $\Delta_{-}$asymptotics, and the right, $\Delta_{+}$. An allowed eigenmode must satisfy these same asymptotics, which is only possible if $\psi_{a, R}^{+}$is proportional to $\psi_{a, L}^{-}$. Using the connection coefficients (3.16) (once again replacing $\left.i \sigma \rightarrow \nu_{a}\right)$, we find that this is true when $\cos \left(\pi \nu_{a}\right)=$ 0 . Throwing out redundant choices, the allowed values of $\nu_{a}$ are $\nu_{a}=\frac{1}{2}+a$ (with $a=$ $0,1,2, \ldots)$, yielding the interface operator spectrum:

$$
\Delta_{a}=\frac{d}{2}+a, \quad a=0,1, \ldots
$$

Of course, above we only considered those operators descending from the bulk field $\phi$. However, at $O(1)$ in the $1 / N$ expansion this is the only bulk field modified by the defect. Boundary primaries built from other fields simply have dimensions of the form $\Delta+n$, with $\Delta$ the dimension of a CFT bulk operator $\mathcal{O}$; these operators are merely descendants $\partial_{y}^{n} \mathcal{O}$, where $y$ is the coordinate transverse to the interface. Only at $O(1 / N)$ does a generic primary $\mathcal{O}$ develop singularities as it is brought to the defect, giving rise to a shift in the 
conformal dimension of the corresponding boundary operator. Of course, there are also the multi-trace operators, whose dimensions in the large $N$ limit are simply the sum of the dimensions of their component operators.

Finally, note that in the above we have chosen the standard quantization for all operators. However, there is one operator which lies in the unitarity window: the operator $\mathcal{O}_{0}$ dual to $\phi_{0}$, which has dimension $\frac{d}{2}$. The corresponding double trace operator has dimension $d$, matching that of the interface displacement operator [19], which can be used to generate deformations in the interface shape. This strongly suggests that this double trace operator should be identified with the displacement operator. Since $\mathcal{O}_{0}$ is the leading boundary operator in the expansion of the bulk operator $\varphi$, this is consistent with the CFT expectation that the displacement operator takes the form $\# \varphi^{2}+\cdots$.

\section{Correlation functions}

With the interface bulk-boundary propagator in hand, we turn now to the computation of CFT observables. This section will deal with the two-point functions. Recall that the bulk field $\phi$ is dual to a boundary operator $\varphi_{+}$of dimension $\Delta_{+}$in $A_{+}$, and to an operator $\varphi_{-}$ of dimension $\Delta_{-}$in $A_{-}$. There are therefore three different correlation functions that we can compute:

$$
\mathcal{G}_{a b}\left(x, x^{\prime}\right)=\left\langle\varphi_{a}(x) \varphi_{b}\left(x^{\prime}\right)\right\rangle \quad a, b= \pm, x \in A_{a}, x^{\prime} \in A_{b} .
$$

We begin in section 4.1 by deriving explicit expressions for these two-point functions from the results of sections 2.2 and 3.3. Section 4.2 uses the conformal block expansion of the two-point function to give an alternate derivation of the spectrum of interface primaries at large $N$.

\subsection{Evaluation of the two-point functions}

Section 2.2 showed how to extract two-point functions from the bulk-boundary propagators. This can be done using the closed form expressions of section 3 , and we do so for $\mathcal{G}_{++}$and $\mathcal{G}_{--}$in section 4.1.1. It is, however, also instructive to work with the representation obtained from solving the dual integral equation, as this approach is more general. To illustrate this procedure, we therefore derive $\mathcal{G}_{-+}$in section 4.1.2 using the integral representation of appendix $\mathrm{B}$.

\subsection{1 $\left\langle\varphi_{+}(x) \varphi_{+}\left(x^{\prime}\right)\right\rangle$ and $\left\langle\varphi_{-}(x) \varphi_{-}\left(x^{\prime}\right)\right\rangle$}

To evaluate the two-point function $\mathcal{G}_{++}$for operator insertions in the $A_{+}$region, recall that in the standard holographic normalization, $\mathcal{G}_{++}=2 \nu\left[K_{+}\right]_{\Delta_{+}}$. The bulk-boundary propagator in Janus frame was given in equation (3.44).

We make our computation in Poincaré patch coordinates on the $H^{d}$ slices, $d s_{H^{d}}^{2}=$ $\frac{d \vec{x}^{2}+d y^{2}}{y^{2}}$, corresponding to a planar interface. We wish to compute the correlation function in a flat conformal frame, which requires including the additional Weyl factor $\left(y y^{\prime}\right)^{-\Delta_{+}}$. 
Combining this factor with equation (2.30a) gives the correlator

$$
\begin{aligned}
\mathcal{G}_{++}\left(x, x^{\prime}\right) & =\frac{2 \nu}{\left(y y^{\prime}\right)^{\Delta_{+}}} \lim _{z \rightarrow 0}[4 z(1-z)]^{-\Delta_{+} / 2} K^{+-}\left(z, x ; x^{\prime}\right) \\
& =\frac{2 \nu}{\left(4 y y^{\prime}\right)^{\Delta_{+}}} \frac{\sin \pi \nu}{\pi} \frac{\Gamma(d / 2)}{\pi^{d / 2}} \xi^{-d / 2}{ }_{2} F_{1}\left(\begin{array}{c}
d / 2,-\nu \\
1-\nu
\end{array} \mid-\frac{1}{\xi}\right) \\
& =\frac{c\left(\Delta_{+}\right)}{\left|x-x^{\prime}\right|^{2 \Delta_{+}}}\left[1+\frac{\sin \pi \nu}{\pi} \frac{\Gamma(d / 2) \Gamma(\nu+1)}{\Gamma\left(\Delta_{+}+1\right)} \xi^{\Delta_{+}}{ }_{2} F_{1}\left(\begin{array}{c}
d / 2, \Delta_{+} \\
\Delta_{+}+1
\end{array} \mid-\xi\right)\right]
\end{aligned}
$$

where $c\left(\Delta_{+}\right)=\frac{2 \nu \Gamma\left(\Delta_{+}\right)}{\pi^{d / 2} \Gamma(\nu)}$ is the standard holographic normalization factor for scalar correlators. For the planar interface, the conformal cross ratio takes the form $\xi=\frac{\left(x-x^{\prime}\right)^{2}}{4 y y^{\prime}}$.

When comparing with CFT we will use the canonically normalized correlation function

$$
G_{++}^{\text {norm. }}\left(x, x^{\prime}\right)=\frac{1}{\left|x-x^{\prime}\right|^{2 \Delta_{+}}}\left[1+B \xi^{\Delta_{+}}{ }_{2} F_{1}\left(\begin{array}{c}
\Delta_{+}, d / 2 \\
\Delta_{+}+1
\end{array} \mid-\xi\right)\right]
$$

with

$$
B=\frac{\Gamma(d / 2) \Gamma(\nu+1)}{\Gamma\left(\Delta_{+}+1\right)} \frac{\sin \pi \nu}{\pi} .
$$

The $\varphi_{-} \varphi_{-}$correlator is obtained from this correlation function by combining the reflection $y \mapsto-y$ together with the replacement $\nu \mapsto-\nu$.

\subsection{2 $\left\langle\varphi_{-}(\chi) \varphi_{+}\left(\chi^{\prime}\right)\right\rangle$}

We evaluate this propagator using the results of appendix B, which are derived in Poincaré patch coordinates $(u, \chi)$ on $H^{d+1}$. The boundary points $\chi$ can be expressed in spherical coordinates with radial coordinate $r$; the interface is located on the sphere $r=R$, and $A_{+}$is in the interior region. The evaluation of this two-point function can be reduced by $\mathrm{SO}(d, 1)$ transformation to the case where $r^{\prime}=0$. Equation (2.30c) tells us we should compute $\left[K_{+}\right]_{\Delta_{-}}$. Due to equation (B.21), as $r^{\prime} \rightarrow 0$ the only harmonic that contributes is $\ell=0$. We will therefore evaluate the $\ell=0$ contribution for $r^{\prime}>0$, and then send $r^{\prime} \rightarrow 0$. (We must perform the process this way: it involves a distributional integral for which the limit does not commute with the integral.)

Set $\ell=0$ and take $r>R$. We take $Y_{0}=1$, in which case $c_{0}=\left(\operatorname{vol} S^{d-1}\right)^{-1}$. Inserting (B.18) into (B.11) and using (B.12) gives

$$
\left[K_{+, \ell=0}\right]_{\Delta_{-}}=c_{0} \frac{\sin \pi \nu}{\pi} \frac{1}{r^{d-2}} \int_{0}^{R} d s \frac{1}{\left(r^{2}-s^{2}\right)^{1-\nu}} \frac{d}{d s}\left[\frac{\theta\left(s-r^{\prime}\right)}{\left(s^{2}-r^{\prime 2}\right)^{\nu}}\right] .
$$

Once we integrate by parts, we can take the limit $r^{\prime} \rightarrow 0$ to obtain

$$
\left[K_{+, \ell=0}\right]_{\Delta_{-}}=2 c_{0} \frac{\sin \pi \nu}{\pi} \frac{1}{r^{d}}\left(\frac{r^{2}}{R^{2}}-1\right)^{\nu},
$$

and using equation (2.30c) gives us the correlator itself,

$$
\mathcal{G}_{-+}(\chi, 0)=\left\langle\varphi_{-}(\chi) \varphi_{+}(0)\right\rangle=\frac{c_{+-}}{r^{2 \Delta_{-}} R^{2 \nu}}\left(1-\frac{R^{2}}{r^{2}}\right)^{\nu} \quad c_{+-}=2 \nu \frac{\sin \pi \nu}{\pi} \frac{\Gamma(d / 2)}{\pi^{d / 2}} .
$$


Now, $\mathrm{SO}(d, 1)$ invariance imples that the two-point function at general $\chi^{\prime}$ can be written in the form ${ }^{7}$

$$
\mathcal{G}_{-+}\left(\chi, \chi^{\prime}\right)=\left(\frac{R}{r^{2}-R^{2}}\right)^{\Delta_{-}}\left(\frac{R}{R^{2}-r^{\prime 2}}\right)^{\Delta_{+}} f(\xi)
$$

with the conformal cross ratio for a spherical defect given by

$$
\xi\left(\chi, \chi^{\prime}\right)=\frac{R^{2}\left(\chi-\chi^{\prime}\right)^{2}}{\left(R^{2}-r^{2}\right)\left(R^{2}-r^{\prime 2}\right)} .
$$

At $\chi^{\prime}=0, \xi=\frac{r^{2}}{R^{2}-r^{2}}$, so $\frac{r^{2}}{R^{2}}=\frac{\xi}{1+\xi}$. We can find $\left[K_{+}\right]_{\Delta_{-}}$at general values of $\chi^{\prime}$ simply by making this replacement in the above expression. (Note that when $r^{\prime}<R<r, \xi<-1$.) Setting $r^{\prime}=0$ and equating (4.10) and (4.9) gives

$$
f(\xi)=c_{+-}(-\xi)^{-d / 2} .
$$

The correlator thus becomes

$$
\left\langle\varphi_{-}(\chi) \varphi_{+}\left(\chi^{\prime}\right)\right\rangle=c_{+-}\left(\frac{R}{r^{2}-R^{2}}\right)^{\Delta_{-}}\left(\frac{R}{R^{2}-r^{\prime 2}}\right)^{\Delta_{+}}(-\xi)^{d / 2}=\frac{c_{+-}}{\left|x-x^{\prime}\right|^{d}}\left(\frac{r^{2}-R^{2}}{R^{2}-r^{\prime 2}}\right)^{\nu} .
$$

If we perform a conformal transformation to planar interface coordinates $x=(\vec{x}, y)$ such that $\Delta_{+}$is the region given by $y>0$, the correlator takes the form

$$
\left\langle\varphi_{-}(x) \varphi_{+}\left(x^{\prime}\right)\right\rangle=\frac{c_{+-}}{\left|x-x^{\prime}\right|^{d}}\left(\frac{-y}{y^{\prime}}\right)^{\nu}=\frac{c_{+-}}{(-2 y)^{\Delta_{-}}\left(2 y^{\prime}\right)^{\Delta_{+}}}(-\xi)^{-d / 2},
$$

where now $\xi=\frac{\left(x-x^{\prime}\right)^{2}}{4 y y^{\prime}}$. For some purposes it is useful to work with the folded picture correlator $\hat{\mathcal{G}}_{-+}$. With $\hat{x}=(\vec{x},-y)=(\vec{x}, \hat{y})$, and $\hat{\xi}=\frac{\left(\hat{x}-x^{\prime}\right)^{2}}{4 \hat{y} y^{\prime}}=-1-\xi$, this is defined by

$$
\hat{\mathcal{G}}_{-+}\left(\hat{x}, x^{\prime}\right)=\left\langle\varphi_{-}(\hat{x}) \varphi_{+}\left(x^{\prime}\right)\right\rangle_{\text {folded }}=\left\langle\varphi_{-}(x) \varphi_{+}\left(x^{\prime}\right)\right\rangle=\frac{c_{+-}}{(2 \hat{y})^{\Delta_{-}}\left(2 y^{\prime}\right)^{\Delta_{+}}}(1+\hat{\xi})^{-d / 2} .
$$

Finally, for comparison with CFT it is useful to give the canonically normalized folded correlator

$$
\hat{G}_{-+}^{\text {norm. }}\left(\hat{x}, x^{\prime}\right)=\sqrt{\frac{\sin \pi \nu}{\pi \nu}} \frac{\Gamma(d / 2)}{\sqrt{\Gamma\left(\frac{d}{2}+\nu\right) \Gamma\left(\frac{d}{2}-\nu\right)}} \frac{(1+\hat{\xi})^{-d / 2}}{(2 \hat{y})^{\Delta_{-}}\left(2 y^{\prime}\right)^{\Delta_{+}}} .
$$

\subsection{Fusion channels and defect spectrum}

Bulk correlation functions in CFT are well known to be completely determined by the structure coefficients in the theory $C_{q r}^{p}$. If $\varphi_{p}$ denote the quasi-primary operators of the theory,

$$
\varphi_{p}(x) \varphi_{p^{\prime}}\left(x^{\prime}\right)=\sum_{q} C_{p p^{\prime}}^{q} C\left[x-x^{\prime} ; \partial_{x^{\prime}}\right] \varphi_{q}\left(x^{\prime}\right)
$$

holds as an operator equation, where $C\left[x-x^{\prime}, \partial_{x^{\prime}}\right]$ are operators depending only on conformal dimension. Inserting this expansion into correlation functions reduces their computation to a knowledge of $C_{p p^{\prime}}^{q}$, which are model-dependent, and conformal blocks, which

\footnotetext{
${ }^{7}$ For a planar defect, the prefactor takes the more familiar form $(-2 y)^{-\Delta_{-}}\left(2 y^{\prime}\right)^{-\Delta_{+}}$.
} 
are universal. The requirement of crossing symmetry - that the answer be independent of the order in which OPEs are taken - puts powerful constraints on the spectrum and couplings of a CFT, and underlies the recent success of the numerical conformal bootstrap methods initiated in [20].

Using the folding trick, any interface can be thought of as a boundary of the product CFT. In the presence of a planar boundary any primary $\varphi_{p}$ has the boundary OPE

$$
\varphi_{p}(x)=\sum_{a} B_{p}^{a} D\left[y ; \partial_{\vec{x}}\right] \psi_{a}(\vec{x})
$$

where $\psi_{a}$ runs over the $\mathrm{SO}(d, 1)$ quasi-primaries living on the boundary, and $D$ is a function depending only on the dimension $\Delta_{a}$. Here we have decomposed $x=(\vec{x}, y)$, with $y$ the distance to the boundary. In the presence of an interface, this expansion can be used to evaluate any bulk object in terms of interface correlators. In particular, interface twopoint functions can be decomposed in terms of boundary conformal blocks, which were first derived in [21]. The requirement that this process yields the same result as the bulk OPE imposes constraints on the CFT and its boundary.

In the presence of an interface, non-trivial constraints arise already at the level of twopoint functions, and so the structure implied by the bulk and boundary OPEs should be realized in the two-point functions $\mathcal{G}_{a b}$. Since at leading order in the $1 / N$ expansion double trace interfaces do not see coupling to any other fields, the conformal block structure at this order should only involve operators realized holographically in terms of the field $\phi$ itself. We will show in this section that the operator dimensions predicted by the conformal block decomposition of the two-point functions match those derived in section 3.4, and so indeed satisfy this condition. Furthermore, we use our results to derive relations between OPE coefficients, which we will compare in specific cases to known CFT results in section 6 .

In what follows we work with the canonically normalized correlation function $G_{a b}$.

\subsubsection{Bulk fusion channel}

We begin with the bulk fusion channel, derived from the OPE as $\xi \rightarrow 0$. The correlator of two scalar bulk operators $O$ and $O^{\prime}$ has the bulk conformal block decomposition [21]

$$
\left\langle O(x) O^{\prime}\left(x^{\prime}\right)\right\rangle_{\mathcal{D}}=\frac{1}{(2 y)^{\Delta}\left(2 y^{\prime}\right)^{\Delta^{\prime}}} \xi^{-\left(\Delta+\Delta^{\prime}\right) / 2} \sum_{q} C_{O O^{\prime}}^{q} B_{q}^{\mathrm{id}} \mathcal{F}\left(\Delta_{q}, \Delta-\Delta^{\prime} \mid \xi\right)
$$

where $q$ runs over bulk quasi-primaries, and the bulk channel conformal block is

$$
\mathcal{F}(\Delta, \delta \mid \xi)=\xi^{\Delta / 2}{ }_{2} F_{1}\left(\begin{array}{c}
\frac{1}{2}(\Delta+\delta), \frac{1}{2}(\Delta-\delta) \\
\Delta+1-\frac{d}{2}
\end{array} \mid-\xi\right) .
$$

When the argument $\delta=0$ we simply omit it. In the case of $2 \mathrm{~d}$ CFT this is the expression for the global conformal block; these are the only blocks that will be visible in our decomposition even in 2d CFT, since Virasoro blocks degenerate to global conformal blocks at large central charge. 
$G_{++}^{\text {norm. }}$. The bulk fusion channel is obtained from an inspection of (4.5). The first term corresponds to the identity block, while the leading behavior of the second term corresponds to an operator of dimension $2 \Delta_{+}$. A closed form for the conformal block decomposition of the second term follows from the formulae of appendix E.1.3,

$$
x^{\Delta_{+}}{ }_{2} F_{1}\left(\begin{array}{c}
d / 2, \Delta_{+} \\
\Delta_{+}+1
\end{array} \mid-x\right)=\sum_{n=0}^{\infty} \frac{(\nu)_{n}(\nu+1)_{n}\left(\Delta_{+}\right)_{n}}{n !\left(\Delta_{+}+1\right)_{n}\left(\Delta_{+}+\nu+n\right)_{n}} \mathcal{F}\left(2 \Delta_{+}+2 n \mid x\right) .
$$

Therefore the $\varphi_{+} \varphi_{+}$OPE contains a quasiprimary $\mathcal{O}_{n}$ with non-vanishing one point function for every dimension $\Delta_{n}=2 \Delta_{+}+2 n(n=0,1, \ldots)$. This result has a straightforward interpretation: the only operators contributing to the exchange channel at this level are double trace operators built from the descendants of $\varphi_{+}$. Such an interpretation is consistent with the fact that the interface is built from only one bulk field $\Phi$. We can be much more precise: at leading order in the $1 / N$ expansion, the OPE coefficients satisfy the relation

$$
C_{\varphi_{+} \varphi_{+}}^{n} B_{n}^{\mathrm{id}}=\frac{\sin \pi \nu}{\pi} \frac{\Gamma(d / 2) \Gamma(\nu+1)}{\Gamma\left(\Delta_{+}+1\right)} \frac{(\nu)_{n}(\nu+1)_{n}\left(\Delta_{+}\right)_{n}}{n !\left(\Delta_{+}+1\right)_{n}\left(\Delta_{+}+\nu+n\right)_{n}} .
$$

The same analysis applies to $G_{--}^{\text {norm. }}$ under $\nu \rightarrow-\nu$.

$G_{-+}^{\text {norm. }}$. To apply the BCFT formulae we work with a planar interface in the folded picture on the upper half plane. Write

$$
\hat{G}_{-+}^{\text {norm. }}\left(x, x^{\prime}\right)=c^{\prime} \frac{\hat{\xi}^{-d / 2}}{(2 y)^{\Delta_{-}}\left(2 y^{\prime}\right)^{\Delta_{+}}} \times \hat{\xi}^{d / 2}(1+\hat{\xi})^{-d / 2}
$$

with $c^{\prime}=\sqrt{\frac{\sin \pi \nu}{\pi \nu}} \frac{\Gamma(d / 2)}{\sqrt{\Gamma\left(\Delta_{+}\right) \Gamma\left(\Delta_{-}\right)}}$, so that

$$
c^{\prime} \hat{\xi}^{d / 2}(1+\hat{\xi})^{-d / 2}=\sum_{q} C_{\varphi_{-} \varphi_{+}}^{q} B_{q}^{\mathrm{id}} \mathcal{F}\left(\Delta_{q}, 2 \nu \mid \hat{\xi}\right) .
$$

Applying (E.9) with $a=\frac{d}{2}, b=c, \alpha=\Delta_{+}, \beta=\delta_{-}, \gamma=\frac{d}{2}+1$ gives the decomposition

$$
\hat{\xi}^{d / 2}(1+\hat{\xi})^{-d / 2}=\sum_{n=0}^{\infty} \frac{\left(\Delta_{+}\right)_{n}\left(\Delta_{-}\right)_{n}}{n !\left(\frac{d}{2}+n\right)_{n}}{ }_{3} F_{2}\left(\begin{array}{c}
\frac{d}{2}, \frac{d}{2}+n,-n \\
\Delta_{+}, \Delta_{-}
\end{array} \mid 1\right) \mathcal{F}(d+2 n, 2 \nu \mid \hat{\xi}) .
$$

This implies that there is a contribution from fusion channels containing operators $O_{n}$ of dimension $\Delta_{n}=d+2 n$.

These have a quite transparent interpretation in terms of the $\varphi_{-} \varphi_{+}$OPE: since $\varphi_{-}$ and $\varphi_{+}$live in different sectors of the product CFT their OPE is non-singular, and clearly closes in terms of the double trace operators built from descendants of $\varphi_{-}$and $\varphi_{+}$. In particular, we can read off the coefficient product

$$
C_{\varphi_{-} \varphi_{+}}^{O_{n}} B_{O_{n}}^{\mathrm{id}}=c^{\prime} .
$$

Obviously, the operator $O_{0}$ can simply be chosen as the normal-ordered coincidence limit $O_{0}=\left(\varphi_{+} \varphi_{-}\right)-($divergence $)$. In this normalization,

$$
B_{O_{0}}^{\mathrm{id}}=c^{\prime} .
$$




\subsubsection{Boundary fusion channel}

The bulk-boundary OPE

$$
\mathcal{O}(x)=\sum_{a} B_{O}^{a} D\left[y, \partial_{\vec{x}}\right] \psi_{a}(\vec{x})
$$

allows bulk operators to be expanded in terms of boundary primary operators $\psi_{a}$ and their descendants, which we take to be orthogonal

$$
\left\langle\psi_{a}(\vec{x}) \psi_{b}\left(\vec{x}^{\prime}\right)\right\rangle=\frac{\mathcal{N}_{a} \delta_{a b}}{\left|x-x^{\prime}\right|^{\Delta_{a}}} .
$$

Inserting this OPE into a two-point function, one can derive the representation [21]

$$
\left\langle O(x) O^{\prime}\left(x^{\prime}\right)\right\rangle=\frac{1}{(2 y)^{\Delta}\left(2 y^{\prime}\right)^{\Delta^{\prime}}} \sum_{a} \mathcal{N}_{a} B_{O}^{a} B_{O^{\prime}}^{a} \mathcal{F}_{\partial}\left(\Delta_{a} \mid \xi\right),
$$

where the boundary channel conformal block $\mathcal{F}_{\partial}$ is given by

$$
\mathcal{F}_{\partial}(\Delta \mid \xi)=\xi^{-\Delta}{ }_{2} F_{1}\left(\begin{array}{c}
\Delta, \Delta-\frac{d}{2}+1 \\
2 \Delta-d+2
\end{array} \mid-\frac{1}{\xi}\right) .
$$

$G_{++}^{\text {norm. }}$. Using the hypergeometric indentity (E.8b), we can write

$$
G_{++}^{\text {norm. }}\left(x, x^{\prime}\right)=\frac{1}{\left(4 y y^{\prime}\right)^{\Delta_{+}}} \frac{\sin \pi \nu}{\pi} \frac{\Gamma(d / 2) \Gamma(\nu)}{\Gamma\left(\Delta_{+}\right)} \xi^{-d / 2}{ }_{2} F_{1}\left(\begin{array}{c}
d / 2,-\nu \\
1-\nu
\end{array} \mid-\frac{1}{\xi}\right),
$$

which is in the appropriate form to apply (4.31). The decomposition follows from the results of appendix E.1.3 and takes the form

$$
G_{++}^{\text {norm. }}\left(x, x^{\prime}\right)=\frac{1}{\left(4 y y^{\prime}\right)^{\Delta_{+}}} \frac{\sin \pi \nu}{\pi} \frac{\Gamma(d / 2) \Gamma(\nu)}{\Gamma\left(\Delta_{+}\right)} \sum_{k=0}^{\infty} \frac{k !(d / 2)_{k}(1+\nu)_{k}}{(2 k) !(1-\nu)_{k}} \mathcal{F}_{\partial}\left(\frac{d}{2}+k \mid \xi\right)
$$

so that we have a contribution from a pair of boundary operators of dimension $\frac{d}{2}+k$ for each $k=0,1, \ldots$ The fusion coefficients are given explicitly by

$$
B_{\varphi_{+}}^{k} B_{\varphi_{+}}^{k} \mathcal{N}_{k}=\frac{\sin \pi \nu}{\pi} \frac{\Gamma(d / 2) \Gamma(\nu)}{\Gamma\left(\Delta_{+}\right)} \frac{k !(d / 2)_{k}(1+\nu)_{k}}{(2 k) !(1-\nu)_{k}} .
$$

This is the same as the boundary operator spectrum found in section 3.4. Note that as we approach the boundary, the dominant contribution comes from a boundary operator $\psi$ of dimension $\frac{d}{2}$,

$$
\varphi_{+}(x) \sim \frac{1}{(2 y)^{\nu}} \psi_{0}(\vec{x})+\cdots
$$

where

$$
\left\langle\psi_{0}(\vec{x}) \psi_{0}\left(\vec{x}^{\prime}\right)\right\rangle_{\mathcal{D}}=\frac{\sin \pi \nu}{\pi \nu} \frac{\Gamma(d / 2) \Gamma(\nu)}{\Gamma\left(\Delta_{+}\right)} \frac{1}{\left|\vec{x}-\vec{x}^{\prime}\right|^{d}} .
$$

As discussed in section 3.4, it is very natural to guess that $\psi \psi$ fuses into the displacement operator,

$$
D(x) \sim: \psi \psi:(x),
$$

which has dimension $d$. In particular, we expect that the displacement operator two-point function is determined at leading order by the $\phi \phi \phi \phi$ four-point function. 
$\underset{-+}{G_{-+}^{\text {norm. }} .}$ Write the folded picture correlator

$$
\begin{aligned}
\hat{G}_{-+}^{\text {norm. }}\left(x, x^{\prime}\right) & =\frac{c^{\prime}}{(2 y)^{\Delta_{-}}\left(2 y^{\prime}\right)^{\Delta_{+}}} \frac{1}{\hat{\xi}^{d / 2}}\left(1+\hat{\xi}^{-1}\right)^{-d / 2} \\
& =\frac{1}{(2 y)^{\Delta_{-}}\left(2 y^{\prime}\right)^{\Delta_{+}}} \sum_{a} B_{\varphi_{-}}^{a} B_{\varphi_{+}}^{a} \mathcal{N}_{a} \mathcal{F}_{\partial}\left(\Delta_{a} \mid \hat{\xi}\right) .
\end{aligned}
$$

Setting $a=\frac{d}{2}, b=c$ in (E.9) we have

$$
(1+\hat{\xi})^{-d / 2}=\sum_{k=0}^{\infty} \frac{(d / 2)_{k}(1)_{k}}{(2 k) !} \hat{\xi}^{-k}{ }_{2} F_{1}\left(\begin{array}{c}
1+k,-k \\
1
\end{array} \mid 1\right){ }_{2} F_{1}\left(\begin{array}{c}
\frac{d}{2}+k, 1+k \\
2+2 k
\end{array} \mid-\hat{\xi}^{-1}\right)
$$

The first hypergeometric function can be evaluated by replacing the " $c$ " parameter 1 by $1+\epsilon$, using Gauss' summation formula, and taking the limit $\epsilon \rightarrow 0$, giving $(-)^{k}$. We therefore obtain

$$
(1+\hat{\xi})^{-d / 2}=\sum_{k=0}^{\infty} \frac{k !(d / 2)_{k}}{(2 k) !}(-)^{k} \mathcal{F}_{\partial}\left(\Delta_{k} \mid \hat{\xi}\right) \quad \Delta_{k}=\frac{d}{2}+k
$$

matching the spectrum derived in 3.4. The fusion coefficients satisfy

$$
B_{\varphi_{+}}^{k} B_{\varphi_{-}}^{k} \mathcal{N}_{k}=c^{\prime}(-)^{k} \frac{k !(d / 2)_{k}}{(2 k) !}
$$

\section{Interface partition function}

We now turn to the computation of the simplest quantum effect of double trace interfaces: the leading contribution to the sphere free energy due to a double trace interface on the equator, at large $N$. In the specific case $d=2$, this quantitiy coincides with the boundary entropy, or $g$ factor [22], of 2d CFT. The defect free energy is the leading non-extensive contribution to the thermal free energy in the expansion in $\beta / L$, where $\beta^{-1}$ is the temperature and $L$ is the length of a very long semi-infinite cylinder. Thus, for example in $2 \mathrm{~d}$ $\mathrm{BCFT}$ one can write

$$
\log Z=\frac{c}{12} \frac{L}{\beta}+\log g+O(\beta / L) .
$$

Computing the overall one-loop correction to the free energy requires both UV and IR regulators. The defect contribution to the free energy, however, can be expressed as the difference of two free energies defined using the same UV regulator, which is a UV finite quantity. Our construction is as follows. Take the bulk theory to be $\mathrm{CFT}_{+} \otimes \mathrm{CFT}_{-}$. Into this theory we can introduce the double trace interface joining CFT + on the left to CFT on the right, and vice versa. Consider the difference $\Delta F$ of the free energy of this theory with the defect, $F_{+\otimes_{-}}^{\mathcal{D}}$, and without the defect, $F_{+\otimes_{-}}$. The bulk contribution to the free energy cancels between these two terms, and so we have

$$
F_{\text {defect }}=\frac{1}{2} \Delta_{F}=\frac{1}{2}\left(F_{+-}+F_{-+}-F_{++}-F_{--}\right) .
$$


Here $F_{a b}$ denotes the free energy of a theory with a single copy of $C F T_{a}$ on the left and $C F T_{b}$ on the right. The $g$ factor is given by

$$
g^{2}=\frac{Z_{C F T_{+} \otimes C F T_{-}}^{\text {defect }}}{Z_{C F T_{+} \otimes C F T_{-}}^{\text {vacuum }}}=\left(\frac{[\operatorname{det} \mathscr{D}]^{++}[\operatorname{det} \mathscr{D}]^{--}}{[\operatorname{det} \mathscr{D}]^{+-}[\operatorname{det} \mathscr{D}]^{-+}}\right)^{-1 / 2}
$$

with $[\operatorname{det} \mathscr{D}]^{a b}$ the functional determinant of $\mathscr{D}=\left(-\square+m^{2}\right)$ with $(a, b)$ boundary conditions. Using

$$
\frac{d}{d m^{2}}[\operatorname{tr} \log \mathscr{D}]^{a b}=\int d^{d+1} X \sqrt{g_{H^{d+1}}} G^{a b}(X, X)
$$

and $\nu^{2}=\frac{d^{2}}{4}+m^{2}$ we find

$$
\frac{d}{d \nu} \log g^{2}=-\nu \int d^{d+1} X \sqrt{g_{H^{d+1}}} \mathscr{H}(X)
$$

where

$$
\mathscr{H}(X)=\lim _{X^{\prime} \rightarrow X}\left(G^{+-}\left(X ; X^{\prime}\right)+G^{-+}\left(X ; X^{\prime}\right)-G^{++}\left(X ; X^{\prime}\right)-G^{--}\left(X ; X^{\prime}\right)\right) .
$$

Since when $\nu=0$ the defect is trivial (and hence $g=1$ ), the value of $g$ is given by the integral $\log g^{2}=\int_{0}^{\nu} d \nu^{\prime} \frac{d}{d \nu^{\prime}} \log g^{2}$.

\subsection{Regulator}

Equation (5.5) is infrared divergent and must be regularized by cutting off the bulk integral. Expressing the metric in the form

$$
d s_{H^{d+1}}^{2}=d \rho^{2}+\sinh ^{2} \rho\left(d \theta^{2}+d \Omega_{d-1}^{2}\right),
$$

we choose the cutoff surface defined by $\rho=\rho_{*}$, which corresponds to computing the CFT partititon function on the sphere. To compute the one-loop contribution of the interface, we need to express the cutoff surface in Janus coordinates [23]. For our purposes the coordinate system

$$
d s_{H^{d+1}}^{2}=\frac{d \tau^{2}}{4 \tau^{2}(1-\tau)}+\frac{1}{\tau} d s_{H^{d}}^{2}, \quad \tau=4 z(1-z)
$$

is useful; note however that the function $\tau(z)$ is 2 -to- 1 and symmetric about $z=1 / 2$. Writing the metric on $H^{d}$ in the form

$$
d s_{H^{d}}^{2}=\frac{d w^{2}}{w(1+w)}+4 w(1+w) d \Omega_{d-1}^{2},
$$

the Poincaré ball coordinates and Janus coordinates are related by

$$
\cosh \rho=\sqrt{\frac{1+2 w}{\tau}} \quad \tanh \rho \sin \theta=\sqrt{\frac{2 w}{1+2 w}} .
$$

The intersection of the cutoff surface with a leaf of given $\tau$ is therefore defined by the relation $w=w_{*}(\tau)$, where

$$
\tau^{1 / 2}=\epsilon\left(1+2 w_{*}\right), \quad \epsilon=\frac{1}{\cosh \rho_{*}} .
$$

Note that $w_{*} \geq 0$, which means that the minimum value of $\tau$ is given by

$$
\tau \geq \tau_{*}=\epsilon^{2} .
$$




\subsection{Sphere free energy and the $g$-factor}

To proceed, we use equation (3.28) to write $\mathscr{H}$ in the form

$$
\begin{aligned}
\mathscr{H}(X)=\frac{1}{2 \nu} \int d \sigma \mathcal{N}_{\sigma}\left(\frac{1}{A^{--}} \Phi_{L}^{+}(z) \Phi_{R}^{-}(z)\right. & -\frac{1}{A^{++}} \Phi_{L}^{-}(z) \Phi_{R}^{+}(z) \\
& \left.-\frac{1}{A^{--}} \Phi_{L}^{+}(z) \Phi_{R}^{+}(z)+\frac{1}{A^{-+}} \Phi_{L}^{-}(z) \Phi_{R}^{-}(z)\right)
\end{aligned}
$$

where

$$
\mathcal{N}_{\sigma}=\left|\Psi_{\sigma, 0}(0)\right|^{2}
$$

Kummer's formulae (3.15) allow us to write this as

$$
\mathscr{H}(X)=\frac{1}{2} \int_{0}^{\infty} d \sigma \mathcal{N}_{\sigma} \sum_{s= \pm} c_{s}\left[\Phi_{L}^{s}(z)\right]^{2}, \quad c_{ \pm}= \pm \frac{\sin \pi \nu}{\cosh \pi \sigma} \frac{1}{4^{ \pm \nu}}\left|\frac{\Gamma\left(\frac{1}{2} \pm \nu+i \sigma\right)}{\Gamma(1 \pm \nu)}\right|^{2} .
$$

The trace now takes the form

$$
\int d^{d+1} X \sqrt{g_{H^{d+1}}} \mathscr{H}(X)=\frac{1}{2} \int_{0}^{\infty} d \sigma \mathcal{N}_{\sigma} \sum_{s= \pm} c_{s} \times \int d^{d+1} X \sqrt{g_{H^{d+1}}}\left(\Phi_{L}^{s}\right)^{2} .
$$

To evaluate the inner integral, note that the integral over $H^{d}$ in Janus coordinates simply gives the regulated volume $\left(\operatorname{vol} H^{d}\right)_{*}$. For $z<\frac{1}{2}$, a quadratic transformation of ${ }_{2} F_{1}$ allows us to express $\Phi_{L}^{ \pm}$in the form

$$
\Phi_{L}^{ \pm}(z)=\tau^{\Delta_{+} / 2}{ }_{2} F_{1}\left(\begin{array}{c}
a_{ \pm}, b_{ \pm} \\
c_{ \pm}
\end{array} \mid \tau\right), \quad \text { with } \quad a_{ \pm}=\left(b_{ \pm}\right)^{*}=\frac{1}{2}\left(\frac{1}{2} \pm \nu+i \sigma\right), \quad c_{ \pm}=1 \pm \nu .
$$

Since the integral is symmetric under $z \mapsto 1-z$, in the above integral we may make the replacement

$$
\int d^{d+1} X \sqrt{g_{H^{d+1}}}\left(\Phi_{L}^{s}\right)^{2} \rightarrow\left(\operatorname{vol} H^{d}\right)_{*} 2 \int_{\tau_{*}}^{1} \frac{d \tau}{2 \tau^{d / 2+1} \sqrt{1-\tau}} \tau^{\Delta_{s}}\left[{ }_{2} F_{1}\left(\begin{array}{c}
a_{s}, b_{s} \\
c_{s}
\end{array} \mid \tau\right)\right]^{2} ;
$$

the factor of 2 is required since $\tau$ only covers half the geometry. Using the identities

$$
\left[{ }_{2} F_{1}\left(\begin{array}{c}
a, b \\
a+b+\frac{1}{2}
\end{array} \mid \tau\right)\right]^{2}={ }_{3} F_{2}\left(\begin{array}{c}
2 a, 2 b, a+b \\
a+b+\frac{1}{2}, 2 a+2 b
\end{array} \mid \tau\right)
$$

and

$$
\begin{aligned}
\int_{\epsilon^{2}}^{1} d \tau \tau^{s \nu-1}(1-\tau)^{1 / 2}{ }_{3} F_{2}\left(\begin{array}{c}
2 a_{s}, 2 b_{s}, \frac{1}{2}+s \nu \\
1+s \nu, 1+2 s \nu
\end{array} \mid \tau\right) \\
=\frac{\Gamma\left(\frac{1}{2}\right) \Gamma(s \nu)}{\Gamma\left(\frac{1}{2}+s \nu\right)}{ }_{3} F_{2}\left(\begin{array}{c}
2 a_{s}, 2 b_{s}, s \nu \\
1+s \nu, 1+2 s \nu
\end{array} \mid 1\right)-\frac{\epsilon^{2 s \nu}}{s \nu}+O\left(\epsilon^{1+s \nu}\right)
\end{aligned}
$$


together with the doubling formula for $\Gamma$, this becomes

$$
\left(\operatorname{vol} H^{d}\right)_{*}\left[4^{s \nu} \frac{\Gamma(s \nu) \Gamma(1+s \nu)}{\Gamma(1+2 s \nu)}{ }_{3} F_{2}\left(\begin{array}{c}
2 a_{s}, 2 b_{s}, s \nu \\
1+s \nu, 1+2 s \nu
\end{array} \mid 1\right)-\frac{\tau_{*}^{s \nu}}{s \nu}+\cdots\right]
$$

with $\cdots$ vanishing as $\epsilon$ (and thus $\tau_{*}$ ) approaches 0 . We obtain

$$
\begin{aligned}
& \int d^{d+1} \sqrt{g_{H^{d+1}}} \mathscr{H}(X)=\left(\operatorname{vol} H^{d}\right)_{*} \sum_{s} \int_{0}^{\infty} d \sigma 2^{2 s \nu-1} \mathcal{N}_{\sigma} c_{s} \\
& \times\left[\begin{array}{c}
\frac{\Gamma(s \nu) \Gamma(1+s \nu)}{\Gamma(1+2 s \nu)}{ }_{3} F_{2}\left(\begin{array}{c}
\frac{1}{2}+s \nu+i \sigma, \frac{1}{2}+s \nu-i \sigma, s \nu \\
1+s \nu, 1+2 s \nu
\end{array} \mid 1\right)-\frac{\epsilon^{2 s \nu}}{s \nu}+\cdots
\end{array}\right] .
\end{aligned}
$$

This expression has two sources of IR divergence. The first is from the volume $\left(\operatorname{vol} H^{d}\right)_{*}$, while the second is due to the term proportional to $\epsilon^{-\nu}$. ( $\left.\operatorname{vol} H^{d}\right)_{*}$ has an expansion (for $d \notin 2 \mathbb{N}$ ) in powers $\epsilon_{*}^{1-d+2 m}, m=0,1,2, \ldots$. Provided $d$ is not an odd integer, the divergences fall into two non-overlapping series, which can presumably be eliminated by counterterms that do not affect the finite part of the trace. Alternatively, we can define the integral with $s=-$ by analytic continuation to $\nu<0$. Either way, the $\epsilon^{-2 \nu}$ divergence can be dropped, and the regularized volume replaced by the standard renormalized hyperbolic volume $\left(\operatorname{vol} H^{d}\right)_{\text {ren. }}$. We do this from now on.

The $\sigma$ integral now takes the form

$$
\begin{gathered}
s C_{1} \frac{\Gamma(s \nu) \Gamma(1+s \nu)}{\Gamma(1+2 s \nu)} \frac{1}{\pi} \int_{0}^{\infty} d \sigma\left|\frac{\Gamma\left(\frac{d-1}{2}+i \sigma\right) \Gamma\left(\frac{1}{2}+i \sigma\right)^{2} \Gamma\left(\frac{1}{2}+s \nu+i \sigma\right)}{\Gamma(d / 2) \Gamma(1+s \nu) \Gamma(2 i \sigma)}\right|^{2} \\
\times{ }_{3} F_{2}\left(\begin{array}{c}
\frac{1}{2}+s \nu+i \sigma, \frac{1}{2}+s \nu-i \sigma, s \nu \\
1+s \nu, 1+2 s \nu
\end{array} \mid 1\right)
\end{gathered}
$$

where $C_{1}=\frac{1}{2} \frac{\Gamma(d / 2)}{(4 \pi)^{d / 2}} \frac{\sin \pi \nu}{\pi}$. We can evaluate the $\sigma$ integral using the results of section E.3. The renormalized volume integral then takes the form

$$
\left(\int \mathscr{H}(X)\right)_{\text {ren }}=C_{1}\left(\operatorname{vol} H^{d}\right)_{\text {ren }} \sum_{s= \pm} s \frac{\Gamma\left(\Delta_{s}\right) \Gamma(s \nu) \Gamma(1+s \nu)}{\Gamma\left(\Delta_{s}+1\right) \Gamma(1+2 s \nu)}{ }_{3} F_{2}\left(\begin{array}{c}
\Delta_{s}, s \nu, 1+s \nu \\
\Delta_{s}+1,1+2 s \nu
\end{array} \mid 1\right) .
$$

Equation (5.24) can be evaluated using the 3 -term ${ }_{3} F_{2}$ relation (E.20) given in the appendix. Combining this with (5.24) and (5.5) gives the value

$$
\frac{d}{d \nu} \log g^{2}=-\frac{\nu \cos \pi \nu}{(4 \pi)^{d / 2}} \frac{\Gamma\left(\Delta_{+}\right) \Gamma\left(\Delta_{-}\right)}{\Gamma\left(\frac{d}{2}+1\right)} \times\left(\operatorname{vol} H^{d}\right)_{\text {ren }}
$$

Under dimensional regularization the volume of $H^{d}$ becomes [24]

$$
\left(\operatorname{vol} H^{d}\right)_{\mathrm{ren}}=\pi^{\frac{d-1}{2}} \Gamma\left(-\frac{d-1}{2}\right)
$$

so that

$$
\frac{d}{d \nu} \log g^{2}=-\nu \frac{\cos \pi \nu}{\cos \frac{\pi d}{2}} \frac{\Gamma\left(\Delta_{+}\right) \Gamma\left(\Delta_{-}\right)}{\Gamma(1+d)}
$$


It is interesting to compare this to the value of the difference between the renormalized action of $\mathrm{CFT}_{+}$and $\mathrm{CFT}_{-}[24]$ :

$$
\frac{d}{d \nu}\left(S_{\mathrm{CFT}_{+}}-S_{\mathrm{CFT}_{-}}\right)=\nu \frac{\sin \pi \nu}{\sin \frac{\pi d}{2}} \frac{\Gamma\left(\Delta_{+}\right) \Gamma\left(\Delta_{-}\right)}{\Gamma(1+d)}
$$

from which one can extract the shift in central charge. It is amusing to speculate that the similarity of these expression may indicate some deeper relation between the change in central charge under RG flow, and the $g$ factor for the corresponding RG defect.

Note that our result diverges as $d$ approaches odd integers, corresponding to a logarithmic divergence with respect to $\epsilon$. This reflects the fact that in odd dimensions, the defect free energy is associated to a conformal anomaly localized on the interface locus [25-27].

Explicit values. As examples, we give explicit expressions in several cases where the $g$ factor has no ambiguities.

$d=2$ :

$$
\frac{d}{d \nu} \log g^{2}=\frac{\pi}{2} \nu^{2} \cot \pi \nu
$$

$d=4:$

$$
\frac{d}{d \nu} \log g^{2}=-\frac{\pi}{4 !} \nu^{2}(1-\nu)^{2} \cot \pi \nu
$$

$d=6$ :

$$
\frac{d}{d \nu} \log g^{2}=\frac{\pi}{6 !} \nu^{2}(1-\nu)^{2}(2-\nu)^{2} \cot \pi \nu
$$

\section{Comparison to field theory results}

In this section we check our bulk results against computations we make directly in the CFT. We are interested in particular in the coefficients appearing in the correlation functions of section 4.1, and in the $g$ factor of section 5.2. We will compute two-point functions for small $\nu$ by means of conformal perturbation theory in section 6.1 , and show they coincide at large $N$ with the results of section 4.1. We further calculate the $g$ factor and several overlaps of the solvable RG interfaces constructed in $d=2$ coset models by Gaiotto in [5]. We will show in section 6.2 that, assuming the higher $\operatorname{spin} / \mathcal{W}$-CFT correspondence of [16], these coincide at large $N$ with our bulk results in two dimensions for all values $0 \leq \nu<1$.

\subsection{Coefficients from conformal perturbation theory}

A check of the coefficients appearing in the correlation functions of section 4.1 can be made against conformal perturbation theory. A CFT can be perturbed by adding a term

$$
\delta S=\kappa \epsilon^{d-\Delta_{\mathcal{O}}} \int d^{d} x \mathcal{O}(x)+S_{\text {c.t. }}
$$

to the Euclidean action, where $\mathcal{O}$ is an operator of conformal dimension $\Delta_{\mathcal{O}}, \kappa$ is a dimensionless coupling constant, and $\epsilon$ is a (scheme-dependent) length scale which we will take to be a position space short-distance cutoff. $S_{\text {c.t. }}$ is the counterterm action arising during 
the renormalization procedure. Correlation functions of (renormalized) local operators $\mathcal{O}_{i}$ of the perturbed CFT can be expressed schematically in terms of the correlation functions of the CFT as

$$
\left\langle\mathcal{O}_{1}\left(x_{1}\right) \cdots \mathcal{O}_{n}\left(x_{n}\right)\right\rangle_{\text {pert }}=\frac{\left\langle\mathcal{O}_{1}\left(x_{1}\right) \cdots \mathcal{O}_{n}\left(x_{n}\right) e^{-\delta S}\right\rangle}{\left\langle e^{-\delta S}\right\rangle} .
$$

For short flows, the right-hand side can be expanded in powers of the renormalized coupling constants.

We are interested in deforming by an operator of the form $\varphi_{-}^{2}$, the normal-ordered product of $\varphi_{-}$with itself, in the case where $\Delta_{\varphi_{-}}=\frac{d}{2}-\nu$ with $0 \leq \nu<1$. When $\nu=0$ the interface is trivial, while small values of $\nu$ give rise to short RG flows. If the CFT has a weakly curved bulk dual, and if $\varphi_{-}$is dual to a bulk scalar appearing in the path integral, then in the large $N$ limit $\varphi_{-}$is a "generalized free field" (see [28] and reference [29] therein). This means that correlation functions factorize into two-point functions by Wick contraction. The conformal dimension of this operator is then given by twice the dimension $\Delta_{-}$of $\varphi_{-}$, making $\varphi_{-}^{2}$ a marginally relevant operator for small values of $\nu$. In the large $N$ limit it is also expected that $\varphi_{-}^{2}$ is the only non-trivial relevant operator in the OPE of $\varphi_{-}^{2}$ with itself. Denote the coefficient of $\varphi_{-}^{2}$ in this OPE by $C$. In the OPE (position-space cut-off) scheme, the beta function corresponding to $\kappa$ of the double trace deformation reads

$$
\beta=\left(d-2 \Delta_{-}\right) \kappa-\frac{1}{2} \mathcal{A}_{d-1} C \kappa^{2}+\mathcal{O}\left(\kappa^{3}\right),
$$

where $\mathcal{A}_{d-1}=\frac{2 \pi^{\frac{d}{2}}}{\Gamma\left(\frac{d}{2}\right)}$ is the volume of $S^{d-1}$. The value of $\kappa$ at the IR fixed point (where $\beta=0)$ is therefore

$$
\kappa=\frac{4 \nu}{\mathcal{A}_{d-1} C},
$$

such that perturbative results in $\kappa$ correspond to perturbative results in $\nu$.

Let us consider a planar interface. Like in section 4.1.1 we will use the coordinates $x=(\vec{x}, y)$ but work in the flat conformal frame. Recall that in section 4.1.1 we compute the correlation function for two scalar insertions $\varphi_{-}$at points $x$ and $x^{\prime}$, whose distance from the interface is denoted $y$ and $y^{\prime}$. To first order in $\kappa$, this correlation function is perturbatively given by

$$
\left\langle\varphi_{-}(x) \varphi_{-}\left(x^{\prime}\right)\right\rangle_{\text {pert }}=\left\langle\varphi_{-}(x) \varphi_{-}\left(x^{\prime}\right)\right\rangle-\kappa \epsilon^{2 \Delta_{-}-d} \int_{y^{\prime \prime}<0} d^{d} x^{\prime \prime}\left\langle\varphi_{-}^{2}\left(x^{\prime \prime}\right) \varphi_{-}(x) \varphi_{-}\left(x^{\prime}\right)\right\rangle .
$$

The integral runs over the half-space $y^{\prime \prime}<0$, which does not include the two points $x$ and $x^{\prime}$. Conformal invariance allows us to take both $x$ and $x^{\prime}$ to lie on the positive $y$ axis. The correlator inside the integral has the form

$$
\left\langle\varphi_{-}^{2}\left(x^{\prime \prime}\right) \varphi_{-}(x) \varphi_{-}\left(x^{\prime}\right)\right\rangle=C^{\prime}\left|x^{\prime \prime}-x\right|^{-2 \Delta_{-}}\left|x^{\prime \prime}-x^{\prime}\right|^{-2 \Delta_{-}},
$$

so that the right-hand side of (6.5) is proportional to the integral

$$
I=\int_{y^{\prime \prime}<0} d^{d} x^{\prime \prime}\left|x^{\prime \prime}-x\right|^{-d}\left|x^{\prime \prime}-x^{\prime}\right|^{-d} .
$$


Using spherical coordinates parallel to the interface, and $z=-y^{\prime \prime}$, we have

$$
I=\int_{0}^{\infty} d z \int d r d \Omega_{d-2} r^{d-2}\left(r^{2}+(z+y)^{2}\right)^{-\frac{d}{2}}\left(r^{2}+\left(z+y^{\prime}\right)^{2}\right)^{-\frac{d}{2}} .
$$

The angular integral yields the volume $\mathcal{A}_{d-2}$ of $S^{d-2}$, while the integral over $r$ is of the form

$$
\int_{0}^{\infty} d r r^{d-2}\left(r^{2}+a^{2}\right)^{-\frac{d}{2}}\left(r^{2}+b^{2}\right)^{-\frac{d}{2}}=\frac{\mathcal{A}_{d-1}}{2 \mathcal{A}_{d-2}} \frac{(a+b)^{1-d}}{a b},
$$

valid for $a, b>0$. For the remaining integral over $z$ we use

$$
\int_{0}^{\infty} d z \frac{2 z+y+y^{\prime}}{\left(2 z+y+y^{\prime}\right)^{d}(z+y)\left(z+y^{\prime}\right)}=\int_{0}^{\infty} \frac{d z}{\left(2 z+y+y^{\prime}\right)^{d}(z+y)}+\left\{y \leftrightarrow y^{\prime}\right\},
$$

with

$$
\int_{0}^{\infty} \frac{d z}{\left(2 z+y+y^{\prime}\right)^{d}(z+y)}=\frac{1}{(2 y)^{d} d}{ }_{2} F_{1}\left(\begin{array}{c}
d, d \\
d+1
\end{array} \mid-\frac{y^{\prime}-y}{2 y}\right) .
$$

Using (6.9), (6.10) and (6.11), (6.7) is

$$
\begin{aligned}
I & =\frac{\mathcal{A}_{d-1}}{2 d}\left[\frac{1}{(2 y)^{d}}{ }_{2} F_{1}\left(\begin{array}{c}
d, d \\
d+1
\end{array} \mid-\frac{y^{\prime}-y}{2 y}\right)+\left\{y \leftrightarrow y^{\prime}\right\}\right] \\
& =\frac{1}{d} \frac{\mathcal{A}_{d-1}}{\left(4 y y^{\prime}\right)^{d / 2}}{ }_{2} F_{1}\left(\begin{array}{c}
d / 2, d / 2 \\
\frac{d}{2}+1
\end{array} \mid-\frac{\left(y^{\prime}-y\right)^{2}}{4 y y^{\prime}}\right) .
\end{aligned}
$$

Combining (6.4), (6.6), (6.12), and restoring the $x$ and $x^{\prime}$ dependence, (6.5) becomes

$$
\left\langle\varphi_{-}(x) \varphi_{-}\left(x^{\prime}\right)\right\rangle_{\text {pert }}=\left|x-x^{\prime}\right|^{-2 \Delta_{-}}\left(1-\frac{\nu}{d} \frac{C^{\prime}}{C} \frac{4}{\left(4 y y^{\prime}\right)^{\frac{d}{2}}}{ }_{2} F_{1}\left(\begin{array}{c}
d / 2, d / 2 \\
\frac{d}{2}+1
\end{array} \mid-\xi\right)\right),
$$

where $\xi=\frac{\left(x-x^{\prime}\right)^{2}}{4 y y^{\prime}}$ is the conformal cross ratio. To first order in $\nu$, this formula coincides with the one obtained in section 4.1.1, which was

$$
\begin{aligned}
G_{--} & =\frac{1}{\left|x-x^{\prime}\right|^{2 \Delta_{-}}}\left[1+B \xi^{\Delta_{-}}{ }_{2} F_{1}\left(\begin{array}{c}
\Delta_{-}, d / 2 \\
\Delta_{-}+1
\end{array} \mid-\xi\right)\right], \\
B & =-\frac{\Gamma(d / 2) \Gamma(1-\nu)}{\Gamma\left(\Delta_{-}+1\right)} \frac{\sin \pi \nu}{\pi}
\end{aligned}
$$

provided that

$$
C=2 C^{\prime}
$$

This relation holds due to the fact that at leading order, $\varphi_{-}$is a generalized free field. Using Wick contraction it is simple to verify that (6.15) is satisfied. Let us illustrate this in the context of the large- $N$ free/Wilson-Fisher interface of the $O(N)$ vector model in $d$ dimensions. ${ }^{8}$ The theory contains $N$ scalar fields $\phi_{1}, \ldots, \phi_{N}$. The scalar field $\varphi_{-}$,

\footnotetext{
${ }^{8}$ The RG interface between the $O(N)$ free and Wilson-Fisher critical points for finite $N$ was investigated in [4].
} 
corresponding up to normalization to the operator $\phi_{i} \phi_{i}$, and the double trace operator $\varphi_{-}^{2}$, corresponding to $\left(\phi_{i} \phi_{i}\right)^{2}$, have the OPEs

$$
\begin{aligned}
& \varphi_{-}(x) \varphi_{-}(0)=x^{-2 \Delta_{-}}+\sqrt{\frac{2}{N}} x^{-\Delta_{-}} \varphi_{-}(0)+\frac{\sqrt{2\left(N^{2}-N+3\right)}}{N} \varphi_{-}^{2}(0)+\ldots, \\
& \varphi_{-}^{2}(x) \varphi_{-}^{2}(0)=x^{-4 \Delta_{-}}+\frac{4 \sqrt{2 N}(N+2)}{N^{2}-N+3} x^{-3 \Delta_{-}} \varphi_{-}(0)+\frac{\sqrt{8}(N+8)}{\sqrt{N^{2}-N+3}} x^{-2 \Delta_{-}} \varphi_{-}^{2}(0)+\ldots,
\end{aligned}
$$

where ellipses stand for omitted irrelevant operators. For $N \rightarrow \infty$, we obtain

$$
C^{\prime}=\sqrt{2}, \quad C=2 \sqrt{2},
$$

in agreement with (6.15).

The two-point function of $\varphi_{+}$can be obtained, to first order in perturbation theory, in a manner analogous to the one just described by perturbing the IR action with the marginally irrelevant operator $\varphi_{+}^{2}$. If the analogous conditions apply for the OPEs of $\varphi_{+}$ and $\varphi_{+}^{2}$, we indeed obtain the result (4.5) (and (4.6)).

To compute the perturbative overlap across the interface, which we will compare with section 4.1.2, we start with two insertions of the operator $\varphi_{-}$on the $y$ axis at positions $y^{\prime}>0$ and $-y<0$. Let the perturbation run over the half space $\left\{x^{\prime \prime} \mid y^{\prime \prime}>0\right\}$, so that

$$
\left\langle\varphi_{-}(-y) \varphi_{-}\left(y^{\prime}\right)\right\rangle_{\text {pert }}=\left\langle\varphi_{-}(-y) \varphi_{-}\left(y^{\prime}\right)\right\rangle-\kappa \epsilon^{2 \Delta_{-}-d} \int_{y^{\prime \prime}>0} d^{d} x^{\prime \prime}\left\langle\varphi_{-}^{2}\left(x^{\prime \prime}\right) \varphi_{-}(-y) \varphi_{-}\left(y^{\prime}\right)\right\rangle
$$

This time we need to cut off the integral over $x^{\prime \prime}$ at radius $\epsilon$ away from $y^{\prime}$. In order to compute this, let us split the integral into two parts: one where the coordinate $y^{\prime \prime}$ is outside of the slab $s_{\epsilon}=\left(y^{\prime}-\epsilon, y^{\prime}+\epsilon\right)$, and one where it is inside the slab.

Outside of the slab we have to compute the integral

$$
\begin{aligned}
I_{\mathrm{out}} & =\int_{y^{\prime \prime} \notin s_{\epsilon}} d^{d} x^{\prime \prime}\left\langle\varphi_{-}^{2}\left(x^{\prime \prime}\right) \varphi_{-}(-y) \varphi_{-}\left(y^{\prime}\right)\right\rangle \\
& =C^{\prime} \mathcal{A}_{d-2} \int_{y^{\prime \prime} \notin s_{\epsilon}} d y^{\prime \prime} \int d r r^{d-2}\left(\left(y^{\prime \prime}+y\right)^{2}+r^{2}\right)^{-\frac{d}{2}}\left(\left(y^{\prime \prime}-y^{\prime}\right)^{2}+r^{2}\right)^{-\frac{d}{2}},
\end{aligned}
$$

for which we use (6.9) again to obtain

$$
I_{\text {out }}=\frac{C^{\prime}}{2} \mathcal{A}_{d-1}\left[\int_{0}^{y^{\prime}-\epsilon} d y^{\prime \prime} \frac{\left(y+y^{\prime}\right)^{1-d}}{\left(y+y^{\prime \prime}\right)\left(y^{\prime}-y^{\prime \prime}\right)}+\int_{y^{\prime}+\epsilon}^{\infty} d y^{\prime \prime} \frac{\left(2 y^{\prime \prime}+y-y^{\prime}\right)^{1-d}}{\left(y^{\prime \prime}+y\right)\left(y^{\prime \prime}-y^{\prime}\right)}\right] .
$$

The first integral in the square brackets evaluates to

$$
\int_{0}^{y^{\prime}-\epsilon} d y^{\prime \prime} \frac{\left(y+y^{\prime}\right)^{1-d}}{\left(y+y^{\prime \prime}\right)\left(y^{\prime}-y^{\prime \prime}\right)}=\frac{1}{\left(y+y^{\prime}\right)^{d}}\left(\log \frac{y^{\prime}}{y}-\log \frac{\epsilon}{y+y^{\prime}-\epsilon}\right) .
$$

In the other integral we can split the integrand and shift $y^{\prime \prime}$, such that

$$
\int_{y^{\prime}+\epsilon}^{\infty} d y^{\prime \prime} \frac{\left(2 y^{\prime \prime}+y-y^{\prime}\right)^{1-d}}{\left(y^{\prime \prime}+y\right)\left(y^{\prime \prime}-y^{\prime}\right)}=\int_{\epsilon}^{\infty}\left(\frac{d z}{\left(2 z+y+y^{\prime}\right)^{d} z}+\frac{d z}{\left(2 z+y+y^{\prime}\right)^{d}\left(z+y+y^{\prime}\right)}\right) .
$$


Using the analogue of (6.11) one has

$$
\begin{aligned}
\int_{\epsilon}^{\infty} \frac{d z}{\left(2 z+y+y^{\prime}\right)^{d} z} & =\frac{1}{(2 \epsilon)^{d} d}{ }_{2} F_{1}\left(\begin{array}{c}
d, d \\
d+1
\end{array} \mid-\frac{y+y^{\prime}}{2 \epsilon}\right) \\
& =-\frac{1}{\left(y+y^{\prime}\right)^{d}}\left(\log \frac{\epsilon}{y+y^{\prime}}+\log 2+\psi(d)+\gamma\right)+\mathcal{O}(\epsilon),
\end{aligned}
$$

where $\psi$ is the digamma function and $\gamma$ is Euler's constant, together with

$$
\begin{aligned}
\int_{\epsilon}^{\infty} \frac{d z}{\left(2 z+y+y^{\prime}\right)^{d}\left(z+y+y^{\prime}\right)} & =\frac{1}{\left(2\left(y+y^{\prime}\right)\right)^{d} d}{ }_{2} F_{1}\left(\begin{array}{c}
d, d \\
d+1
\end{array} \mid \frac{1}{2}\right) \\
& =\frac{1}{2\left(y+y^{\prime}\right)^{d}}\left(\psi\left(\frac{d+1}{2}\right)-\psi\left(\frac{d}{2}\right)\right),
\end{aligned}
$$

such that the contribution from outside the slab becomes

$$
I_{\text {out }}=\frac{C^{\prime} \mathcal{A}_{d-1}}{2\left(y+y^{\prime}\right)^{d}}\left(\log \frac{y^{\prime}}{y}-2 \log \frac{\epsilon}{y+y^{\prime}}-\log 2+\frac{1}{2} \psi\left(\frac{d+1}{2}\right)-\frac{1}{2} \psi\left(\frac{d}{2}\right)-\psi(d)-\gamma\right) .
$$

Inside the slab we must cut off the integral over the directions parallel to the interface at an appropriate distance from the $y$ axis, depending on the value of $y^{\prime \prime}$. Rescaling integration variables by $y+y^{\prime}$, we have

$$
\begin{aligned}
I_{\text {in }} & =\int_{y^{\prime \prime} \in s_{\epsilon}} d^{d} x^{\prime \prime}\left\langle\varphi_{-}^{2}\left(x^{\prime \prime}\right) \varphi_{-}(-y) \varphi_{-}\left(y^{\prime}\right)\right\rangle= \\
& =\frac{C^{\prime} \mathcal{A}_{d-2}}{\left(y+y^{\prime}\right)^{d}} \int_{-\epsilon^{\prime}}^{\epsilon^{\prime}} d \eta \int_{\sqrt{\left(\epsilon^{\prime}\right)^{2}-\eta^{2}}}^{\infty} d r r^{d-2}\left(\eta^{2}+r^{2}\right)^{-\frac{d}{2}}\left((\eta+1)^{2}+r^{2}\right)^{-\frac{d}{2}},
\end{aligned}
$$

where $\eta$ is the rescaled $y^{\prime \prime}$, and $\epsilon^{\prime}$ is the rescaled cut-off. As $\eta$ is very small, we can expand the last factor of the integrand. All odd powers of $\eta$ will drop out in the integration, so that we can write

$$
I_{\text {in }}=\frac{2 C^{\prime} \mathcal{A}_{d-2}}{\left(y+y^{\prime}\right)^{d}} \int_{0}^{\epsilon^{\prime}} d \eta \int_{\sqrt{\left(\epsilon^{\prime}\right)^{2}-\eta^{2}}}^{\infty} \frac{r^{d-2} d r}{\left(\eta^{2}+r^{2}\right)^{\frac{d}{2}}\left(1+r^{2}\right)^{\frac{d}{2}}}\left(1+\mathcal{O}\left(\eta^{2}\right)\right) .
$$

Changing coordinates to $\tau^{2}=\eta^{2}+r^{2}$ and expanding the factor $\left(1+r^{2}\right)=\left(1+\tau^{2}-\eta^{2}\right)$ in $\eta$ again, this expression can be written as

$$
I_{\text {in }}=\frac{2 C^{\prime} \mathcal{A}_{d-2}}{\left(y+y^{\prime}\right)^{d}} \int_{0}^{\epsilon^{\prime}} d \eta \int_{\epsilon^{\prime}}^{\infty} d \tau \tau^{-2}\left(1-\frac{\eta^{2}}{\tau^{2}}\right)^{\frac{d-3}{2}}\left(\tau^{2}+1\right)^{-\frac{d}{2}}\left(1+\mathcal{O}\left(\eta^{2}\right)\right) .
$$

We now employ the binomial series

$$
\left(1-\frac{\eta^{2}}{\tau^{2}}\right)^{\frac{d-3}{2}}=\sum_{k=0}^{\infty}\left(\begin{array}{c}
\frac{d-3}{2} \\
k
\end{array}\right)(-1)^{k} \frac{\eta^{2 k}}{\tau^{2 k}}
$$

which is valid on the domain of integration. Note that the $\eta$ integral of the $k^{\text {th }}$ term of the sum yields a suppression by $\epsilon^{\prime 2 k+1}$, while its leading contribution to the $\tau$ integral is

$$
\begin{aligned}
\int_{\epsilon^{\prime}}^{\infty} \frac{d \tau}{\tau^{2+2 k}\left(\tau^{2}+1\right)^{\frac{d}{2}}} & =\frac{\left(\epsilon^{\prime}\right)^{-(d+2 k+1)}}{(d+2 k+1)}{ }_{2} F_{1}\left(\begin{array}{c}
d / 2, d / 2+k \\
\frac{d+3}{2}+k
\end{array} \mid-\frac{1}{\left(\epsilon^{\prime}\right)^{2}}\right) \\
& =\frac{1}{\left(\epsilon^{\prime}\right)^{2 k+1}}\left(\frac{1}{2 k+1}+\mathcal{O}\left(\epsilon^{\prime}\right)\right) .
\end{aligned}
$$


We therefore find

$$
I_{\text {in }}=\frac{2 C^{\prime} \mathcal{A}_{d-2}}{\left(y+y^{\prime}\right)^{d}} \sum_{k=0}^{\infty}\left(\begin{array}{c}
\frac{d-3}{2} \\
k
\end{array}\right) \frac{(-1)^{k}}{(2 k+1)^{2}}+\mathcal{O}\left(\epsilon^{\prime}\right) .
$$

For the sum we have

$$
\sum_{k=0}^{\infty}\left(\begin{array}{c}
\frac{d-3}{2} \\
k
\end{array}\right) \frac{(-1)^{k}}{(2 k+1)^{2}}=\frac{\sqrt{\pi}}{4} \frac{\Gamma\left(\frac{d-1}{2}\right)}{\Gamma\left(\frac{d}{2}\right)}\left(\gamma+\log 4+\psi\left(\frac{d}{2}\right)\right),
$$

and thus

$$
I_{\text {in }}=\frac{C^{\prime} \mathcal{A}_{d-1}}{2\left(y+y^{\prime}\right)^{d}}\left(\gamma+\log 4+\psi\left(\frac{d}{2}\right)\right)+\mathcal{O}\left(\epsilon^{\prime}\right) .
$$

Combining the contributions $I_{\text {out }}$ and $I_{\text {in }}$, and using the identity

$$
\psi(d)-\frac{1}{2} \psi\left(\frac{d+1}{2}\right)-\frac{1}{2} \psi\left(\frac{d}{2}\right)=\log 2
$$

together with the value (6.4) of the coupling constant in the IR, the value of the perturbed correlation function (6.18) becomes

$$
\left\langle\varphi_{-}(-y) \varphi_{-}\left(y^{\prime}\right)\right\rangle_{\mathrm{pert}}=\frac{1}{\left(y+y^{\prime}\right)^{d}}+\frac{2 C^{\prime}}{C} \frac{\nu}{\left(y+y^{\prime}\right)^{d}}\left(2 \log \frac{\epsilon}{\left(y+y^{\prime}\right)}+\log \frac{y}{y^{\prime}}\right) .
$$

This expression still contains a divergence in $\epsilon$, which is eliminated by an appropriate counterterm. Conformal invariance dictates that to first order in $\nu$, the correlation function must take the form

$$
\left\langle\varphi_{-}(-y) \varphi_{+}\left(y^{\prime}\right)\right\rangle=\frac{f_{\nu}(\hat{\xi})}{y^{\Delta_{-}}\left(y^{\prime}\right)^{\Delta_{+}}}
$$

where $\hat{\xi}+1=\left(x-x^{\prime}\right)^{2} /\left(4 y y^{\prime}\right)=\left(y+y^{\prime}\right)^{2} /\left(4 y y^{\prime}\right)$ is the conformal cross ratio. Since the case $\nu=0$ corresponds to the identity interface, the function $f_{\nu}(\hat{\xi})$ must satisfy $f_{0}(\hat{\xi})=$ $(1+\hat{\xi})^{-\frac{d}{2}}$. Expanding (6.36) to first order in $\nu$ and using the dimensions $\Delta_{ \pm}=\frac{d}{2} \pm \nu$ leads to the condition

$$
\frac{2 C^{\prime}}{C}\left(2 \log \frac{\epsilon}{y+y^{\prime}}+\log \frac{y}{y^{\prime}}\right)+\text { c.t. }=\left.\frac{\left(y+y^{\prime}\right)^{d}}{\left(y y^{\prime}\right)^{\frac{d}{2}}} \partial_{\nu} f_{\nu}(\hat{\xi})\right|_{\nu=0}+\log \frac{y}{y^{\prime}},
$$

where "c.t." stands for the counterterm contribution. We observe that the condition $C=2 C^{\prime}$ leads to the cancellation of the $\log y / y^{\prime}$ term on both sides. The remaining part of the left-hand side must then be a function of $\hat{\xi}$ alone. In the OPE scheme the counterterm can only depend on the distance $y+y^{\prime}$ of the two field insertions, and therefore cannot do anything other than precisely eliminate the logarithmic divergence. We therefore conclude that

$$
\left.\partial_{\nu} f_{\nu}(\hat{\xi})\right|_{\nu=0}=0
$$

which makes (6.36) indeed agree with the gravitational result (4.16). 


\subsection{Checks from minimal model holography in $d=2$}

In $d=2$, the duality between Vasiliev Higher Spin theory in the bulk and Minimal Model CFTs on the boundary belongs to the best-understood examples of non-supersymmetric holography [16, 30]. The classical bulk contains one massless field of spin $s$ for every integer $s \geq 2$, which transform under the higher spin algebra hs $(\nu)$, depending on (the square of) an a priori arbitrary complex number $\nu$. The theory includes a complex scalar field (with a propagating degree of freedom, unlike the topological higher spin fields) of mass $m^{2}=\nu^{2}-1$, with $-1<\nu<1$. A single higher spin gravity gives rise to two boundary theories: under $\nu \mapsto-\nu$ the algebra hs $(\nu)$ remains unchanged, while in the unitary window the scalar field of dimension $\Delta_{+}$acquires the alternate quantization $\Delta_{-}$.

The asymptotic quantum symmetry algebra $\mathcal{W}_{\infty}(\nu)$ associated to hs $(\nu)$ also arises as the 't Hooft limit of the algebra $\mathcal{W}_{N, k}$. This is the chiral algebra of the CFT $\mathcal{M}_{N, k}$ based on the coset

$$
\frac{\mathfrak{s u}(N)_{k} \otimes \mathfrak{s u}(N)_{1}}{\mathfrak{s u}(N)_{k+1}}
$$

which has central charge

$$
c_{N, k}=(N-1) \frac{k}{N+k} \frac{k+2 N+1}{k+N+1} .
$$

The 't Hooft limit takes $N, k \rightarrow \infty$ at fixed $\nu=\frac{N}{N+k} \cdot{ }^{9}$ Irreducible representations of the coset (6.39) are labelled by a pair $\Lambda=\left(\lambda^{+}, \lambda^{-}\right)$of representation labels of $\mathfrak{s u}(N)_{k}$ and $\mathfrak{s u}(N)_{k+1}$, respectively. ${ }^{10}$ We will only consider charge-conjugate theories with diagonal modular invariant: i.e., the theory contains only left-right symmetric pairs of representations, $\Lambda \otimes \tilde{\Lambda}$ with $\Lambda \simeq \tilde{\Lambda}$, and for each such pair in the Hilbert space, the charge conjugate pair $\bar{\Lambda} \otimes \tilde{\Lambda}$ is present as well. Despite the left-right symmetry, we write tildes over rightmovers for the purpose of clarity.

The large-level limit of such theories is in general a rather subtle issue $[33,34]$ and leads to continuous orbifold theories [35-37]. However, the equivalence with the $\mathcal{W}_{\infty}$ algebras in fact holds for finite $N$ and $k$ - and therefore finite $c$ - since an extension of level-rank duality identifies $\mathcal{W}_{N, k} \cong \mathcal{W}_{\infty}\left(\frac{N}{k+N}\right)$ if $c=c_{N, k}[30,38]$.

For the unitary theories (where $N$ and $k$ are positive integers) there exists a wellknown relevant deformation of the CFT $\mathcal{M}_{N, k}$ which has $\mathcal{M}_{N, k-1}$ as its IR fixed point [39]. Gaiotto introduced interfaces corresponding to this RG flow and gave a recipe for computing its UV-IR overlaps in [5]. The renormalization group flow from $\mathcal{M}_{N, k}$ to $\mathcal{M}_{N, k-1}$ was proposed in [16] to be the double trace flow from $\mathrm{CFT}_{-}$to $\mathrm{CFT}_{+}$, and the one-loop computations of [40] support this proposal. Therefore, we expect Gaiotto's interface to be realized holographically as a double trace interface.

Because the bulk scalar field is complex, we must take care to include additional factors of 2 when comparing $\log g$ and the overlap coefficients with our bulk computations.

\footnotetext{
${ }^{9}$ For other coset models and their RG flows in the 't Hooft limit see e.g. [31, 32].

${ }^{10}$ Here we follow the common convention to suppress an $\mathfrak{s u}(N)_{1}$ representation label, which is automatically fixed by the choice of $\lambda^{+}$and $\lambda^{-}$. Our conventions for $\mathfrak{s u}(N)$ can be found in appendix F.
} 


\subsubsection{RG interface construction at finite $N$ and $k$}

Before we come to the results in the 't Hooft limit let us briefly explain how the interface is constructed at finite (positive integer) $N$ and $k$. We give the interface as a boundary condition in the folded theory $\mathrm{CFT}_{\mathrm{UV}} \otimes \overline{\mathrm{CFT}}_{\mathrm{IR}}$. The chiral algebra of the folded theory is

$$
\frac{\mathfrak{s u}(N)_{k} \otimes \mathfrak{s u}(N)_{1}}{\mathfrak{s u}(N)_{k+1}} \otimes \frac{\mathfrak{s u}(N)_{k-1} \otimes \mathfrak{s u}(N)_{1^{\prime}}}{\mathfrak{s u}(N)_{k}},
$$

where we distinguish the IR copy of the level 1 algebra from the UV copy by a prime. The Hilbert spaces of the UV and IR theories decompose into products of representations $\Lambda_{i} \otimes \tilde{\Lambda}_{i}$, where $\Lambda_{i}$ and $\tilde{\Lambda}_{i}(i=\mathrm{UV}, \mathrm{IR})$ are representations of the left- and rightmoving chiral algebra respectively. The Hilbert space of the folded theory will then contain the product of representations $\Lambda_{U V} \otimes \overline{\tilde{\Lambda}}_{I R}$ for the left-moving and $\tilde{\Lambda}_{U V} \otimes \bar{\Lambda}_{I R}$ for the right-moving degrees of freedom.

The boundary condition corresponding to the RG interface consists of a projection in the $\mathfrak{s u}(N)_{k}$ sector, a permutation brane in the $\mathfrak{s u}(N)_{1}$ sectors, and a Cardy state in the sector $\mathfrak{s u}(N)_{k-1} / \mathfrak{s u}(N)_{k+1}$ of (6.41) [3]. The projection can be implemented by the topological interface [5]

$$
\mathcal{I}=\sum_{\tilde{\lambda}^{+}, \lambda^{-}} \sum_{\lambda} \frac{1}{S_{0 \lambda}^{(k)}} \Pi_{\left(\lambda, \lambda^{-}\right)_{U V} \otimes\left(\tilde{\lambda}^{+}, \lambda\right)_{I R}}
$$

of the product theory. Here $S_{0 \lambda}^{(k)}$ is a modular $S$ matrix entry of the $\mathfrak{s u}(N)_{k}$ WZW model. The operators $\Pi$ project onto the subscript representation ${ }^{11}\left(\lambda, \lambda^{-}\right)_{U V} \otimes\left(\tilde{\lambda}^{+}, \lambda\right)_{I R}$, which are the products of UV and IR representations sharing a common label $\lambda$ of $\mathfrak{s u}(N)_{k}$. When summed over $\lambda$, these operators implement the isomorphism ${ }^{12}$ [41]

$$
\left\{\tilde{\lambda}^{+}, \lambda^{-}\right\} \cong \bigoplus_{\lambda}\left(\lambda, \lambda^{-}\right)_{U V} \otimes\left(\tilde{\lambda}^{+}, \lambda\right)_{I R},
$$

where the left-hand side denotes a representation of the diagonal coset

$$
\frac{\mathfrak{s u}(N)_{k-1} \otimes \mathfrak{s u}(N)_{1} \otimes \mathfrak{s u}(N)_{1}}{\mathfrak{s u}(N)_{k+1}} .
$$

In particular, this isomorphism identifies the $\mathfrak{s u}(N)_{k}$ current operators $J^{(k) a}=J^{(k-1) a}+$ $J^{\left(1^{\prime}\right) a}$ of the two copies of $\mathfrak{s u}(N)_{k}$ in the product theory.

The boundary condition corresponding to the RG interface is given by the fusion product of the topological interface $\mathcal{I}$ with the boundary state

$$
\left.\| B\rangle\rangle=\sum_{\left\{\tilde{\lambda}^{+}, \lambda^{-}\right\}}^{\prime} \sqrt{S_{0 \tilde{\lambda}^{+}}^{(k-1)} \bar{S}_{0 \lambda^{-}}^{(k+1)}}\left|\left\{\tilde{\lambda}^{+}, \lambda^{-}\right\}\right\rangle\right\rangle_{\mathbb{Z}_{2}} .
$$

The prime in this expression indicates that the sum only runs over representations $\left\{\tilde{\lambda}^{+}, \lambda^{-}\right\}$ where the (suppressed) labels of the two $\mathfrak{s u}(N)_{1}$ parts are identical. The Ishibashi states

\footnotetext{
${ }^{11}$ Only the left-moving degrees of freedom are indicated here.

${ }^{12}$ Notice that two representation labels of $\mathfrak{s u}(N)_{1}$ are suppressed on both sides in equation (6.43).
} 
$\left.\left|\left\{\tilde{\lambda}^{+}, \lambda^{-}\right\}\right\rangle\right\rangle_{\mathbb{Z}_{2}}$ are defined such that they implement a permutation (indicated by the subscript $\left.\mathbb{Z}_{2}\right)$ of these $\mathfrak{s u}(N)_{1}$ parts [42-44].

The prescription for computing the operator overlaps is therefore as follows. Suppose we want to compute the overlap of the UV operator $\Phi^{U V}$ and the conjugate of the IR operator $\Phi^{I R}$, which are composed of left- and right-moving parts

$$
\Phi^{U V}=\phi^{U V} \tilde{\phi}^{U V}, \quad \Phi^{I R}=\phi^{I R} \tilde{\phi}^{I R} .
$$

The operators $\phi^{U V} \tilde{\phi}^{I R}$ and $\tilde{\phi}^{U V} \phi^{I R}$ then constitute the left- and right-moving part of the corresponding operator in the doubled theory, respectively. If $\phi^{U V}$ is an operator in the representation $\left(\lambda^{+}, \lambda^{-}\right)$, and $\tilde{\phi}^{I R}$ is in the representation conjugate to $\left(\tilde{\lambda}^{+}, \tilde{\lambda}^{-}\right)$, we write $\phi^{U V} \tilde{\phi}^{I R}$ as a state in the representation $\left\{\tilde{\lambda}^{+}, \lambda^{-}\right\}$of the left-hand side of (6.43). This image only exists if the representation labels of $\mathfrak{s u}(N)_{k}$ agree, i.e. if $\lambda^{+}=\tilde{\lambda}^{-}$.

After the projection we compute the inner product of $\phi^{U V} \tilde{\phi}^{I R}$ and the $\mathbb{Z}_{2}$ flipped image of $\tilde{\phi}^{U V} \phi^{I R}$, where the latter is obtained by exchanging all degrees of freedom of $\mathfrak{s u}(N)_{1}$ and $\mathfrak{s u}(N)_{1^{\prime}}$. This requires that the (suppressed) representation labels of $\mathfrak{s u}(N)_{1}$ and $\mathfrak{s u}(N)_{1^{\prime}}$ agree. Finally, the resulting inner product must be multiplied with the corresponding coefficient of the boundary state, leading to the formula

$$
\left\langle\Phi^{U V} \Phi^{I R} \mid R G\right\rangle=\frac{\sqrt{S_{0 \tilde{\lambda}^{+}}^{(k-1)} \bar{S}_{0 \lambda^{-}}^{(k+1)}}}{S_{0 \lambda}^{(k)}}\left\langle\phi_{\left(\lambda, \lambda^{-}\right)}^{U V} \tilde{\phi}_{\left(\tilde{\lambda}^{+}, \lambda\right)}^{I R} \mathbb{Z}_{2}\left(\tilde{\phi}_{\left(\lambda, \lambda^{-}\right)}^{U V} \phi_{\left(\tilde{\lambda}^{+}, \lambda\right)}^{I R}\right)\right\rangle
$$

\subsubsection{The RG interface in the 't Hooft limit}

For finite $N$ and $k$, one way to quantify the length of an RG flow is to consider the reflectivity of the RG interface [3]. Reflectivity is measured here with respect to specific parts of the chiral symmetry algebra, and different definitions exist. A coefficient which exists for any conformal interface measures reflection and transmission of energy and momentum [45]. From the matrix

$$
R=\frac{1}{\langle 0 \mid R G\rangle}\left(\begin{array}{c}
\left\langle T^{U V} \tilde{T}^{U V} \mid R G\right\rangle\left\langle T^{U V} T^{I R} \mid R G\right\rangle \\
\left\langle\tilde{T}^{U V} \tilde{T}^{I R} \mid R G\right\rangle\left\langle T^{I R} \tilde{T}^{I R} \mid R G\right\rangle
\end{array}\right)=:\left(\begin{array}{cc}
R_{11} & R_{12} \\
R_{21} & R_{22}
\end{array}\right)
$$

one defines the reflection and transmission coefficients

$$
\mathcal{R}=\mathcal{N}^{-1}\left(R_{11}+R_{22}\right), \quad \mathcal{T}=\mathcal{N}^{-1}\left(R_{12}+R_{21}\right),
$$

where

$$
\mathcal{N}=\sum_{i, j} R_{i j}=\frac{c_{N, k}+c_{N, k-1}}{2}
$$

These coefficients have the property that $\mathcal{R}+\mathcal{T}=1$. Also, $0 \leq \mathcal{R} \leq 1$ for interfaces between unitary CFTs, with $\mathcal{R}=0$ for topological interfaces and $\mathcal{R}=1$ for interfaces which are (totally reflective) conformal boundary states.

For our RG interfaces, the matrix $R$ of (6.48) is rather easy to compute. The (leftmoving) energy-momentum tensor components of the UV and the IR are given by

$$
T^{U V}=T^{(k)}+T^{(1)}-T^{(k+1)}, \quad T^{I R}=T^{(k-1)}+T^{\left(1^{\prime}\right)}-T^{(k)},
$$


where

$$
T^{(k)}=\frac{1}{k+N} \sum_{a}: J^{(k) a} J^{(k) a}:
$$

is the standard Sugawara energy momentum tensor of the $\mathfrak{s u}(N)_{k}$ WZW model. Following the prescription of identifying $J^{(k) a}=J^{(k-1) a}+J^{\left(1^{\prime}\right) a}$ and applying the $\mathbb{Z}_{2}$ transformation $J^{(1) a} \leftrightarrow J^{\left(1^{\prime}\right) a}$ one obtains [3]

$$
\begin{aligned}
R_{11} & =\frac{N^{2}-1}{2(k+N)^{2}} \frac{k+2 N+1}{k+N+1}=\frac{1}{2} \nu^{2}(1+\nu)+\mathcal{O}\left(\frac{1}{k}, \frac{1}{N}\right), \\
R_{12}=R_{21} & =\frac{(N-1)(k-1)(k+2 N+1)}{2(k+N)^{2}} \\
& =\frac{N}{2}\left(1-\nu^{2}\right)-\frac{1}{2}\left(1+\nu^{2}\right)+\mathcal{O}\left(\frac{1}{k}, \frac{1}{N}\right), \\
R_{22} & =\frac{N^{2}-1}{2(k+N)^{2}} \frac{k-1}{k+N-1}=\frac{1}{2} \nu^{2}(1-\nu)+\mathcal{O}\left(\frac{1}{k}, \frac{1}{N}\right) .
\end{aligned}
$$

We observe that in the 't Hooft limit, the entries $R_{11}$ and $R_{22}$ (related to reflection) remain finite, while the off-diagonal entries $R_{12}$ and $R_{21}$ (related to transmission) diverge. The coefficients $\mathcal{R}$ and $\mathcal{T}$, however, remain finite, and asymptote to $\mathcal{R}=0$ and $\mathcal{T}=1$, as for a topological interface. Notice that in spite of the finite change

$$
c_{N, k}-c_{N, k-1}=\frac{2(N-1) N(N+1)}{(N+k-1)(N+k)(N+k+1)}=2 \nu^{3}+\mathcal{O}\left(k^{-2}, N^{-2}\right)
$$

in central charge, there is in fact no contradiction here, since the central charges of the UV and the IR theory are both infinite in the 't Hooft limit. The RG interface in the 't Hooft limit is in general not the identity, as shown by the non-trivial boundary entropy computed in section 5.2 and confirmed in the next subsection.

One could at this point also compute the overlaps - the matrix $R$ - for higher spin fields $W_{s}$ instead of $T$. Each higher spin field of the bulk corresponds to a descendent of the vacuum representation of the boundary CFT. In the coset numerator theory, the state corresponding to the field of spin $s$ has the form [46]

$$
\left|W_{s}\right\rangle=\sum_{n=0}^{s} A_{n} s_{a_{1} \ldots a_{n} b_{1} \ldots b_{s-n}} J_{-1}^{(k) a_{1}} \cdots J_{-1}^{(k) a_{n}} J_{-1}^{(\ell) b_{1}} \cdots J_{-1}^{(\ell) b_{s-n}}|0\rangle,
$$

where $s_{c_{1} \ldots c_{s}}$ is proportional to the totally symmetric invariant tensor of rank $s$ present for $2 \leq s \leq N$ in $\mathfrak{s u}(N)$. The coefficients $A_{n}$ are determined by requiring that $\left|W_{s}\right\rangle$ transforms trivially under the denominator subalgebra, and by the normalization condition $\left\langle W_{s} \mid W_{s}\right\rangle=c / s$. For the example $s=3$ one finds

$$
\begin{array}{ll}
A_{0}=\eta k(k+N)(2 k+N), & A_{1}=-3 \eta(k+N)(N+1)(2 k+N), \\
A_{2}=3 \eta(k+N)(N+1)(N+2), & A_{3}=-\eta(N+1)(N+2), \\
\eta=\left(\frac{N}{18\left(N^{2}-4\right)(N+1)^{2}(N+2)(k+N)^{2}(2 k+N)(k+N+1)^{2}(2 k+3 N+2)}\right)^{\frac{1}{2}}
\end{array}
$$


which leads to the overlap matrix

$$
\begin{aligned}
R_{11} & =-\frac{\left(N^{2}-1\right)(N+2)(k+2 N+1)}{3(k+N)^{2}(2 k+N)(N+k+1)}=-\frac{\nu^{3}(\nu+1)}{3(2-\nu)}+\mathcal{O}\left(\frac{1}{k}, \frac{1}{N}\right) \\
R_{12} & =R_{21}=\sqrt{\frac{(2 k+N-2)(2 k+3 N+2)}{(2 k+N)(2 k+3 N)}} \frac{(N-1)(k-1)(k+2 N+1)}{3(k+N)^{2}} \\
& =\frac{N}{3}\left(1-\nu^{2}\right)+\frac{3 \nu^{4}-5 \nu^{2}-4}{3\left(4-\nu^{3}\right)}+\mathcal{O}\left(\frac{1}{k}, \frac{1}{N}\right) \\
R_{22} & =\frac{\left(N^{2}-1\right)(N+2)(k-1)}{3(k+N)^{2}(2 k+3 N)(k+N-1)}=\frac{\nu^{3}(1-\nu)}{3(2+\nu)}+\mathcal{O}\left(\frac{1}{k}, \frac{1}{N}\right) .
\end{aligned}
$$

The fact that $R_{11}$ is negative for unitary theories is an indication that the conformal RG interface breaks the higher spin algebra. Also, the four entries do not sum up to $\left(c_{N, k}+c_{N, k-1}\right) / 3$, such that they do not provide a sensible measure of reflection and transmission.

\subsubsection{RG interface boundary entropy}

In the boundary state formalism, the $g$ factor of the RG interface is the coefficient of the vacuum Ishibashi state in the defect boundary state, i.e.,

$$
g^{2}=\frac{S_{00}^{(k+1)} S_{00}^{(k-1)}}{\left(S_{00}^{(k)}\right)^{2}} .
$$

The modular $S$ matrix elements of the right-hand side can be found in the standard literature (see e.g. [47]), and are reproduced for convenience in appendix F. We observe that the $g$ factor can be written as a product

$$
g^{2}=\mathcal{P}_{1} \mathcal{P}_{2}
$$

with

$$
\begin{aligned}
& \mathcal{P}_{1}=\left(\frac{(k+N)^{2}}{(k+N+1)(k+N-1)}\right)^{\frac{N-1}{2}}, \\
& \mathcal{P}_{2}=\prod_{m=1}^{N-1}\left(\frac{\sin \left(\frac{\pi m}{k+N+1}\right) \sin \left(\frac{\pi m}{k+N-1}\right)}{\sin ^{2}\left(\frac{\pi m}{k+N}\right)}\right)^{N-m} .
\end{aligned}
$$

In the 't Hooft limit, the logarithm of $\mathcal{P}_{1}$ only contributes at subleading order in $1 / N$,

$$
\log \mathcal{P}_{1}=-\frac{N-1}{2}\left[\log \left(1+\frac{1}{k+N}\right)+\log \left(1-\frac{1}{k+N}\right)\right]=\frac{\nu^{2}}{2 N}+\mathcal{O}\left(N^{-2}\right) .
$$

In order to compute the logarithm of $\mathcal{P}_{2}$, define

$$
x=\frac{\pi m}{k+N}, \quad \delta x=\frac{\pi}{k+N} .
$$


The following expansion in $\delta x$ holds:

$$
\log \left[\frac{\sin \left(\frac{x}{1+\delta x / \pi}\right)}{\sin x} \frac{\sin \left(\frac{x}{1-\delta x / \pi}\right)}{\sin x}\right]=\left(2 x \cot x-\frac{x^{2}}{\sin ^{2} x}\right) \frac{\delta x^{2}}{\pi^{2}}+\mathcal{O}\left(\delta x^{4}\right) .
$$

With (6.62) and (6.63) we can express the leading contribution to $\log \mathcal{P}_{2}$ for large $k$ and $N$ as

$$
\log \mathcal{P}_{2}=\sum_{m=1}^{N}\left(\nu-\frac{x}{\pi}\right)\left(2 x \cot x-\frac{x^{2}}{\sin ^{2} x}\right) \frac{\delta x}{\pi}+\mathcal{O}\left(\frac{\nu^{2}}{N^{2}}\right) .
$$

The sum is convergent as long as every $x$ is smaller than $\pi$, which means that the expansion is valid in the case $0 \leq \nu<1$. In the 't Hooft limit the sum becomes an integral. Since the error term is of order $1 / N^{2}$, the sum will yield the correction up to first order in $1 / N$. By the Euler-Maclaurin formula we obtain

$$
\begin{aligned}
\log \mathcal{P}_{2} & =\frac{1}{\pi} \int_{0}^{\pi \nu}\left(\nu-\frac{x}{\pi}\right)\left(2 x \cot x-\frac{x^{2}}{\sin ^{2} x}\right) d x+\frac{\nu^{2}}{2 N}+\mathcal{O}\left(N^{-2}\right) \\
& =\frac{1}{\pi^{2}} \int_{0}^{\pi \nu} x^{2} \cot x d x+\frac{\nu^{2}}{2 N}+\mathcal{O}\left(N^{-2}\right) \quad(\nu<1) .
\end{aligned}
$$

Combining the results (6.61) and (6.65) we find that

$$
g^{2}=\mathcal{P}_{1} \mathcal{P}_{2}=\exp \left[\pi \int_{0}^{\nu} \lambda^{2} \cot (\pi \lambda) d \lambda+\frac{\nu^{2}}{N}+\mathcal{O}\left(N^{-2}\right)\right]
$$

In the Hooft limit we therefore have

$$
\frac{d}{d \nu} \log g^{2}=\pi \nu^{2} \cot (\pi \nu)
$$

After including the factor of 2 for the complex field, this agrees precisely with the bulk result (5.29).

\subsubsection{Matching of coefficients for two-point functions}

We can also use the recipe of section 6.2.2 to check the coefficients in the two-point functions of section 4.1. The bulk scalar field is dual on the IR side of the interface to the CFT operator $\varphi_{+}=\Phi_{(f, 0)}^{I R}$, and to $\varphi_{-}=\Phi_{(0, f)}^{U V}$ on the UV side, where $f$ denotes the fundamental representation of $\mathfrak{s u}(N)$. The conformal dimensions of $\Phi_{(f, 0)}^{I R}$ and $\Phi_{(0, f)}^{U V}$ for finite $N$ and $k$ are

$$
\Delta_{(f, 0)}^{I R}=\frac{N-1}{N}\left(1+\frac{N+1}{N+k}\right), \quad \Delta_{(0, f)}^{U V}=\frac{N-1}{N}\left(1-\frac{N+1}{N+k+1}\right) .
$$

The first coefficient we would like to match is the constant $B$ in (4.5). Writing the OPE of the scalar field in the IR as $\Phi_{(f, 0)}^{I R} \times \Phi_{(f, 0)}^{I R} \sim 1+C_{I R}^{\prime} \Phi_{(\text {adj }, 0)}^{I R}$, the constant $B$ is given by the expression

$$
B=C_{I R}^{\prime} g^{-1}\left\langle\Phi_{(\mathrm{adj}, 0)}^{I R} \mathrm{id}^{U V} \mid R G\right\rangle .
$$


The operator $\Phi_{(\text {adj,0) }}^{I R}$ corresponds to the double trace perturbation in the IR.

For finite $N$ and $k$, the value of $C_{I R}^{\prime}$ can be obtained, e.g., from Coulomb gas methods $[48,49]$. Since this calculation is not in the focus of this paper we refrain from performing it here, and only point out that in the 't Hooft limit the OPE coefficients of UV and IR coincide, with $C^{\prime}$ approaching 1 . The coefficient $C$ goes to 2 , in agreement with condition (6.15).

Now consider the overlap of the IR operator $\Phi_{(\operatorname{adj}, 0)}^{I R}$ with the identity in the UV. In the numerator $\mathfrak{s u}(N)_{k-1} \otimes \mathfrak{s u}(N)_{1^{\prime}}$ of the IR coset, the chiral state corresponding to this operator can be written as

$$
\left|\phi_{(\operatorname{adj}, 0)}^{I R}\right\rangle=\frac{1}{\sqrt{\mathcal{N}}}\left(J_{-1}^{(k-1) a}-(2 N+k-1) J_{-1}^{\left(1^{\prime}\right) a}\right)\left|J_{a}^{(k-1)}\right\rangle,
$$

with normalization constant

$$
\mathcal{N}=\left(N^{2}-1\right)(2 N+k-1)(2 N+k) .
$$

In (6.70), a sum over the indices $a$ of the currents is implied, and indices are raised and lowered with the Killing form $K^{a b}$. The state $\left|J_{a}^{(k-1)}\right\rangle$ is the corresponding Virasoro highestweight state present in the $\mathfrak{s u}(N)_{k-1}$ adjoint representation.

Following the recipe of section 6.2.1 we compute the overlap

$$
\left\langle\left(\mathrm{id}^{U V} \tilde{\phi}_{(\mathrm{adj}, 0)}^{I R}\right) \mathbb{Z}_{2}\left(\tilde{\mathrm{id}}^{U V} \phi_{(\operatorname{adj}, 0)}^{I R}\right)\right\rangle
$$

by replacing $J_{-1}^{1}$ by $J_{-1}^{1^{\prime}}$ in the $\mathbb{Z}_{2}$-flipped state. This yields

$$
\left\langle\left(\operatorname{id}^{U V} \tilde{\phi}_{(\operatorname{adj}, 0)}^{I R}\right) \mathbb{Z}_{2}\left(\phi_{(\operatorname{adj}, 0)}^{I R} \tilde{\mathrm{id}}^{U V}\right)\right\rangle=\frac{1}{2 N+k}=\frac{1-\nu}{1+\nu} \frac{1}{k} .
$$

The Ishibashi state coefficient is

$$
\frac{\sqrt{S_{\mathrm{adj}, 0}^{(k-1)} S_{00}^{(k+1)}}}{S_{00}^{(k)}} .
$$

Its computation is similar to that of the $g$ factor in the section above. Using equation (F.1) of the appendix we find that

$$
\frac{S_{\mathrm{adj}, 0}^{k-1} S_{0,0}^{k+1}}{\left(S_{0,0}^{k}\right)^{2}}=g^{2} \times \frac{\left.\sin \left(\frac{\pi(N+1)}{k+N-1}\right) \sin \left(\frac{\pi(N-1)}{k+N-1}\right)\right)}{\left(\sin \left(\frac{\pi}{k+N-1}\right)\right)^{2}},
$$

where we used the expression for the $g$ factor from the previous section. In the 't Hooft limit, the right-most factor goes as

$$
\frac{\sin \left(\frac{\pi(N+1)}{k+N-1}\right) \sin \left(\frac{\pi(N-1)}{k+N-1}\right)}{\left(\sin \left(\frac{\pi}{k+N-1}\right)\right)^{2}}=\frac{k^{2} \sin ^{2}(\pi \nu)}{\pi^{2}(1-\nu)^{2}}+\mathcal{O}(k) .
$$

The two factors (6.72) and (6.74) therefore combine into

$$
\begin{aligned}
\left\langle\Phi_{(\operatorname{adj}, 0)}^{I R} \mathrm{id}^{U V} \mid R G\right\rangle & =g \frac{\sqrt{\sin \left(\frac{\pi(N+1)}{k+N-1}\right) \sin \left(\frac{\pi(N-1)}{k+N-1}\right)}}{\sin \left(\frac{\pi}{k+N-1}\right)} \frac{1}{2 N+k} \\
& =\frac{g}{\pi} \sin (\pi \nu) \frac{1}{1+\nu}+\mathcal{O}\left(k^{-1}, N^{-1}\right),
\end{aligned}
$$


and we therefore have

$$
B=\frac{\sin (\pi \nu)}{\pi(1+\nu)} .
$$

Comparing with (4.6) we observe that we have a precise match.

Choosing the two insertions to be on the UV side can be done in the analogous way, and in the limit merely results in the replacement $\nu \mapsto-\nu$.

It is also straightforward to verify the overlap of the scalar across the interface we found in section 4.1.2. In the UV theory, the chiral part of the scalar $\varphi_{-}$, corresponding to $\Phi_{(0, f)}^{U V}$, can be written as a state in the numerator of the UV coset as

$$
\left|\phi_{(0, f)}^{U V}\right\rangle=\left|\omega_{1}\right\rangle^{(1)}
$$

where $\omega_{1}$ denotes the first fundamental weight (which is the highest weight in the fundamental representation) of $\mathfrak{s u}(N)$, and $\left|\omega_{1}\right\rangle^{(1)}$ is the highest weight state of the fundamental representation of $\mathfrak{s u}(N)_{1}$. In order to have a non-vanishing overlap we insert the conjugate of the scalar $\varphi_{+}$in the IR, corresponding to $\Phi_{(\bar{f}, 0)}^{I R}$. The chiral state in the IR coset numerator lies in the product $\bar{f}^{(k-1)} \otimes f^{\left(1^{\prime}\right)}$ of the antifundamental representation of $s u\left(N_{k-1}\right)$ and the fundamental representation of $s u\left(N_{1^{\prime}}\right)$. It is given by

$$
\left|\phi_{(\bar{f}, 0)}^{I R}\right\rangle=\frac{1}{\sqrt{N}} \sum_{i=1}^{N}(-1)^{i}\left|\omega_{N-1}-\alpha_{N-1}-\ldots-\alpha_{i}\right\rangle^{(k-1)}\left|\omega_{1}-\alpha_{1}-\ldots-\alpha_{i-1}\right\rangle^{\left(1^{\prime}\right)},
$$

where $\omega_{N-1}$ is the highest weight of the antifundamental representation, $\alpha_{i}$ are the simple roots of $\mathfrak{s u}(N)$, and $|\lambda\rangle^{(k)}$ denotes the basis state of weight $\lambda$ in the fundamental (or antifundamental) representation of $\mathfrak{s u}(N)_{k}$. For finite $N$ and $k$, the overlap coefficient of one scaler field insertion on each side of the interface is

$$
\left\langle\Phi_{(0, f)}^{U V} \Phi_{(f, 0)}^{I R} \mid R G\right\rangle=\frac{\sqrt{S_{0 f}^{(k-1)} S_{0 f}^{(k+1)}}}{S_{00}^{(k)}}\left\langle\left(\phi_{(0, f)}^{U V} \tilde{\phi}_{(\bar{f}, 0)}^{I R}\right) \mathbb{Z}_{2}\left(\tilde{\phi}_{(0, f)}^{U V} \phi_{(\bar{f}, 0)}^{I R}\right)\right\rangle .
$$

In the prefactor of modular $S$ matrices we notice that for any level $k$,

$$
\frac{S_{0 f}^{(k)}}{S_{00}^{(k)}}=\prod_{m=1}^{N-1} \frac{\sin \frac{\pi(m+1)}{N+k}}{\sin \frac{\pi m}{N+k}}=\frac{\sin (\pi \nu)}{\sin (\pi \nu / N)}=\left(\frac{\sin (\pi \nu)}{\pi \nu} N+\mathcal{O}\left(N^{-1}\right)\right) .
$$

Using the explicit expressions (6.79) and (6.80), the other factor in (6.81) becomes

$$
\left\langle\left(\phi_{(0, f)}^{U V} \tilde{\phi}_{(\bar{f}, 0)}^{I R}\right) \mathbb{Z}_{2}\left(\tilde{\phi}_{(0, f)}^{U V} \phi_{(\bar{f}, 0)}^{I R}\right)\right\rangle=\frac{1}{N} .
$$

The RG overlap in the 't Hooft limit is therefore

$$
\left\langle\Phi_{(0, f)}^{U V} \Phi_{(f, 0)}^{I R} \mid R G\right\rangle=g \frac{\sin (\pi \nu)}{\pi \nu} .
$$

Dividing by $g=\langle 1\rangle$ and including a factor of 2 for the complex scalar, this is indeed what we obtain as coefficient from (4.16). 


\section{Conclusions and discussion}

In this paper, we gave a semi-classical holographic construction of double trace interfaces - RG interfaces associated to an RG flow initiated by double trace deformation. We discussed methods for constructing double trace interfaces of any shape and computing observables using mixed boundary value problem techniques. We gave a simple integral representation for the bulk Green's function associated to a spherical interface, as well as the bulk-boundary propagators and CFT two-point correlation functions in closed form. From these results we obtained the leading contribution of the spherical defect to the CFT partition function (yielding for $d=2$ the boundary entropy).

Double trace interfaces have arisen previously in concrete systems of interest, allowing us to test our gravitational results against CFT computations. We derived the two-point function in the presence of double trace interfaces in conformal perturbation theory, and showed that the result matches the weak-coupling limit of our gravitational computation in the large- $N$ limit, where the single trace operator becomes a generalized free field. This result generalizes the special case of a Wilson-Fisher/free field interface near $d=4$, studied in [4] using bootstrap methods. It would be interesting to compute the correlator at large $N$ in the most physically relevant dimension $d=3$. This should be doable by standard methods; we leave this to future work.

In $d=2$, the $\mathcal{W}_{N}$ minimal model RG defects constructed in [5] are realized as double trace interfaces within the higher spin gravity/WCFT proposal of [16]. Using our results, we were able to compute several interface overlap coefficients in the semi-classical limit. We computed the same coefficients using the exact results of [5] and showed that they coincide at large $N$. Furthermore, we computed the exact boundary $g$-factor in these models, and showed that its large $N$ limit is reproduced by our one-loop gravitational result.

Questions and future directions. There are several further observables associated to double trace interfaces that would be interesting to compute. One is the leading (oneloop) correction to the stress tensor two-point function (which in $d=2$ reduces to the transmission/reflection coefficients of [45]) and other operators, and the leading (classical) contribution to the higher-point functions.

A further question, of interest for the theory of conformal interfaces, would be to study the fusion of double trace interfaces. This computation was outlined in section 2.3.

There are two further general points of possible interest we would like to mention. The first is related to defect conformal bootstrap. For free fields, the work of [50] showed that the two-point function for Dirichlet and Neumann boundary conditions of a free field could be reproduced by imposing crossing symmetry. We constructed the large- $N$ spectrum of non-trivial defect operators, and saw that the conformal block decomposition of our twopoint functions closes on these operators in the boundary channel, and on double trace operators in the bulk channel. It is interesting to ask whether our two-point functions are the unique solution to the crossing equations that can be generated in this way at large $N$; it is further possible that, using this boundary spectrum as a starting point, one could push the analytic bootstrap results of [4] past leading order in $\epsilon$. It is also tempting to 
apply Mellin bootstrap [51] methods to this problem, since there the effects of double trace operators are included automatically in the Mellin space representation.

The second point is that the match between the gravitational partition function and the $g$-factor for Gaiotto's defect provides further evidence for the proposal of [16] that Zamolodchikov's integrable RG flow is implemented holographically as a double trace deformation. The starting-point of this RG flow is described on the one hand by the alternative quantization, but according to the original duality it should be described also by a higher spin gravity with the standard quantization but (at finite $N$ ) a slightly different value of $\nu$. This suggests a duality between distinct higher spin gravity theories. It was shown in [40] that the one-loop correction to the central charge is also consistent with this hypothesis. It would be of interest to pursue this question further.

\section{Acknowledgments}

We thank Shouvik Datta for collaboration on early stages of this work. CSC would like to thank Ilka Brunner and Enrico Brehm for discussions. CMT wishes to acknowledge the support of the Thousand Young Talents Program and Fudan University.

\section{A Coordinate systems on hyperbolic space}

We word exclusively in Euclidean signature in this paper, so we are concerned with hyperbolic space $H^{d+1}$ in $d+1$ dimensions.

\section{A.1 Standard coordinates}

The standard Poincaré patch metric for hyperbolic space of unit radius is

$$
d s_{H^{d+1}}^{2}=\frac{d u^{2}+d \chi^{i} d \chi^{i}}{u^{2}} .
$$

It is also natural for us to work with spherical defects, in which case it is helpful to use radial coordinates on the flat boundary:

$$
d s_{H^{d+1}}^{2}=\frac{d u^{2}+d r^{2}+r^{2} d \Omega_{d-1}^{2}}{u^{2}} .
$$

The boundary of $H^{d+1}$ is located at $u=0$.

We also work with the Poincaré ball model, whose metric is

$$
d s^{2}=d \rho^{2}+\sinh ^{2} \rho d s_{S^{d}}^{2} .
$$

The defining function is $2 e^{-\rho}$, and the conformal boundary is the sphere located at $\rho \rightarrow \infty$.

\section{A.2 Janus coordinates}

The Janus decomposition is a slicing of $H^{d+1}$ by surfaces whose geometry is $H^{d}$. The standard form of the Janus decomposition is

$$
d s_{H^{d+1}}^{2}=\frac{1}{\cos ^{2} \mu}\left(d \mu^{2}+d s_{H^{d}}^{2}\right) .
$$


In this coordinate system, the boundary is split into two components, located at $\mu= \pm \frac{\pi}{2}$. The interface between the two boundary components is located at the boundary of $H^{d}$.

We work rather in terms of the following coordinate systems:

$$
\sinh \beta=\tan \mu, \quad z=\frac{1}{2}(1-\sin \mu)
$$

in terms of which

$$
\begin{aligned}
d s_{H^{d+1}} & =\frac{1}{4 z^{2}(1-z)^{2}}\left(d z^{2}+z(1-z) d s_{H^{d}}^{2}\right) \\
& =d \beta^{2}+\cosh ^{2} \beta d s_{H^{d}}^{2} .
\end{aligned}
$$

Defining function. Choosing a member of the conformal class of metrics on the boundary is equivalent to choosing a defining functional $\zeta$ with the following properties.

1. In a neighborhood $U$ of the boundary, $\zeta$ vanishes on the boundary but nowhere else.

2. In $U$, the metric can be written in Fefferman-Graham form

$$
d s^{2}=\frac{d \zeta^{2}+\gamma_{i j}(\zeta, x) d x^{i} d x^{j}}{\zeta^{2}}
$$

where $\left(\zeta, x^{i}\right)$ form a coordinate system, and $\lim _{\zeta \rightarrow 0} \gamma_{i j}(\zeta, x)$ yields a non-degenerate metric for all $x$.

The defining function adapted to Janus coordinates is $\zeta=\sqrt{4 z(1-z)}$, inducing the boundary metric $d s_{\partial M}^{2}=d s_{H^{d}}^{2}$. Note that $\zeta$ is not a coordinate at $z=\frac{1}{2}$ since it is invariant under $z \mapsto 1-z$, but it is a good coordinate in a neighborhood of either boundary component.

\section{A.3 Coordinates on $H^{d}$}

We use the following coordinate systems for the $H^{d}$ slices of the Janus geometry. The standard Poincaré patch and ball metrics are

$$
d s_{H^{d}}^{2}=\frac{d y^{2}+d \vec{x}^{2}}{y^{2}}=\frac{4\left(d \hat{r}^{2}+\hat{r}^{2} d \Omega_{d-1}^{2}\right)}{\left(1-\hat{r}^{2}\right)^{2}} .
$$

Rather than $\hat{r}$, we find the following coordinate more useful:

$$
w=\frac{\hat{r}^{2}}{1-\hat{r}^{2}},
$$

in terms of which

$$
d s_{H^{d}}^{2}=\frac{d w^{2}}{w(1+w)}+4 w(1+w) d \Omega_{d-1}^{2} .
$$




\section{B Bulk-boundary propagator from dual integral equations}

The purpose of this section is to explicitly derive an expression for the mixed bulk-boundary correlator by solving a mixed boundary value problem. The setup is as follows: the interface is the sphere $|\chi|=R$, which separates the CFT + region in the interior from the CFT - region of the exterior. Throughout this section we use the Poinaré patch coordinates $(u, \chi)$.

Here we compute the mixed bulk-boundary propagator $K_{+}\left(u, \chi ; \chi^{\prime}\right)$ for $\left|\chi^{\prime}\right|<R$. We will do so by imposing properties [K1]-[K3] in order. Property [K1] can be satisfied by expanding $K_{+}$in solutions to the wave equation with an unspecified coefficient function $\psi$,

$$
\begin{aligned}
K_{+}\left(u, \chi ; \chi^{\prime}\right) & =\sum_{\ell} Y_{\ell}\left(\hat{\chi}, \hat{\chi}^{\prime}\right) \mathcal{K}_{\ell}\left(u, r ; r^{\prime}\right) \\
\mathcal{K}_{\ell}\left(u, r ; r^{\prime}\right) & =\frac{1}{r^{d / 2-1}} \int_{0}^{\infty} d \xi \psi_{\ell}(\xi) u^{d / 2} K_{\nu}(\xi u) J_{m_{\ell}}(\xi r) .
\end{aligned}
$$

Here $m_{\ell}=\ell+\frac{d}{2}-1, r=|\chi|$, and $\hat{\chi}=\frac{\chi}{r}$ is the unit vector in the $\chi$ direction. This expansion is in terms of boundary spherical waves $r^{1-d / 2} Y_{\ell}\left(\hat{\chi}, \hat{\chi}^{\prime}\right) J_{m_{\ell}}(\xi r)$ of Laplacian eigenvalue $\xi^{2}$, in which $Y_{\ell}\left(\hat{\chi}, \hat{\chi}^{\prime}\right)$ denotes the spherical harmonic rotationally symmetric around the $\hat{\chi}^{\prime}$ axis with Laplacian eigenvalue $-\ell(\ell+d-2)(\ell=0,1, \ldots)$. Using

$$
\left[u^{d / 2} K_{\nu}(\xi u)\right]_{\Delta_{-}}=a_{0} \xi^{-\nu} \quad\left[u^{d / 2} K_{\nu}(\xi u)\right]_{\Delta_{+}}=b_{0} \xi^{\nu} \quad a_{0}=2^{\nu-1} \Gamma(\nu) \quad b_{0}=-\frac{\Gamma(1-\nu)}{\nu 2^{\nu+1}}
$$

we obtain the asymptotics of $\mathcal{K}$ as $u \rightarrow 0$,

$$
\begin{aligned}
& {\left[\mathcal{K}_{\ell}\right]_{\Delta_{-}}=\frac{a_{0}}{r^{d / 2-1}} \int_{0}^{\infty} d \xi \xi^{-\nu} J_{m_{\ell}}(\xi r) \psi_{m_{\ell}}(\xi)} \\
& {\left[\mathcal{K}_{\ell}\right]_{\Delta_{+}}=\frac{b_{0}}{r^{d / 2-1}} \int_{0}^{\infty} d \xi \xi^{\nu} J_{m_{\ell}}(\xi r) \psi_{m_{\ell}}(\xi) .}
\end{aligned}
$$

Property $[\mathrm{K} 2]$ says that $[\mathcal{K}]_{\Delta_{+}}$should vanish when $r>R$. This can be guaranteed by imposing the ansatz

$$
\psi_{\ell}(\xi)=\xi \int_{0}^{R} d s g_{\ell}(s) J_{m_{\ell}-\nu}(\xi s),
$$

a fact verified as follows. Since $\nu<1$, this integral exists provided $g_{m}(s)$ is bounded on $[0, R]$. Using the relation

$$
\frac{d}{d s}\left[s^{\lambda} J_{\lambda}(\xi s)\right]=\xi s^{\lambda} J_{\lambda-1}(\xi s)
$$

we see that

$$
\left[\mathcal{K}_{\ell}\right]_{\Delta_{+}}=\frac{b_{0}}{r^{d / 2-1}} \int_{0}^{R} \frac{d s}{s^{m_{\ell}+1-\nu}} g_{\ell}(s) \frac{d}{d s}\left[s^{m_{\ell}-\nu+1} \int_{0}^{\infty} d \xi \xi^{\nu} J_{m_{\ell}}(\xi r) J_{m_{\ell}-\nu+1}(\xi s)\right] .
$$

The inner integral is a Weber-Schafheitlin discontinuous integral (see e.g. (11.4.33) of [52]), and takes the value

$$
\int_{0}^{\infty} d \xi \xi^{\nu} J_{m_{\ell}}(\xi r) J_{m_{\ell}-\nu+1}(\xi s)=\frac{2^{\nu}}{\Gamma(1-\nu)} \frac{s^{\nu-m_{\ell}-1} r^{m_{\ell}}}{\left(s^{2}-r^{2}\right)^{\nu}} \theta(s-r) .
$$


We thus find

$$
\left[\mathcal{K}_{\ell}\right]_{\Delta_{+}}=\frac{2^{\nu} b_{0}}{\Gamma(1-\nu)} r^{\ell} \int_{0}^{R} \frac{d s}{s^{\ell+d / 2-\nu}} g_{\ell}(s) \frac{d}{d s}\left[\frac{\theta(s-r)}{\left(s^{2}-r^{2}\right)^{\nu}}\right] .
$$

In particular, $\left[\mathcal{K}_{\ell}\right]_{\Delta_{+}}$vanishes for $r>R$, and so our ansatz guarantees that [K2] is satisfied.

We must also impose the condition $\left[\mathcal{K}_{\ell}\right]_{\Delta_{-}}=\delta^{(d)}\left(x-x^{\prime}\right)$ for $r<R$. Inserting our ansatz gives

$$
\left[\mathcal{K}_{\ell}\right]_{\Delta_{-}}=\frac{a_{0}}{r^{d / 2-1}} \int_{0}^{R} d s g_{\ell}(s) \int_{0}^{\infty} d \xi \xi^{1-\nu} J_{m_{\ell}}(\xi r) J_{m_{\ell}-\nu}(\xi s) .
$$

Once again the inner integral is a Weber-Schafheitlin discontinous integral, and takes the value

$$
\int_{0}^{\infty} d \xi \xi^{1-\nu} J_{m_{\ell}}(\xi r) J_{m_{\ell}-\nu}(\xi s)=\frac{1}{a_{0}} \frac{s^{m_{\ell}-\nu} r^{-m_{\ell}}}{\left(r^{2}-s^{2}\right)^{1-\nu}} \theta(r-s)
$$

so that for $r<R$,

$$
\left[\mathcal{K}_{\ell}\right]_{\Delta_{-}}=\frac{1}{r^{\ell+d-2}} \int_{0}^{r} d s g_{\ell}(s) \frac{s^{m_{\ell}-\nu}}{\left(r^{2}-s^{2}\right)^{1-\nu}} .
$$

Let us now impose the condition that $\left[\mathcal{K}_{\ell}\right]_{\Delta_{-}}=\phi_{\ell}(r)$, i.e.,

$$
\int_{0}^{r} d s g_{\ell}(s) \frac{s^{m_{\ell}-\nu}}{\left(r^{2}-s^{2}\right)^{1-\nu}}=r^{\ell+d-2} \phi_{\ell}(r) .
$$

This is an integral equation of Abel type. For $0<\alpha<1$, the equation

$$
\int_{0}^{r} \frac{f(t) d t}{[h(r)-h(t)]^{\alpha}}=g(r)
$$

has solution

$$
f(s)=\frac{\sin (\pi \alpha)}{\pi} \frac{d}{d s} \int_{0}^{s} \frac{h^{\prime}(t) g(t) d u}{[h(s)-h(t)]^{1-\alpha}}
$$

(see e.g. (2.3.2) of [17]). With the substitutions

$$
\alpha \mapsto 1-\nu \quad h(t) \mapsto t^{2} \quad f(s) \mapsto s^{m_{\ell}-\nu} g_{\ell}(s) \quad g(r) \mapsto r^{\ell+d-2} \phi_{\ell}(r)
$$

we obtain

$$
g_{\ell}(s)=\frac{2}{s^{m_{\ell}-\nu}} \frac{\sin (\pi \nu)}{\pi} \frac{d}{d s} \int_{0}^{s} \frac{u^{\ell+d-1} \phi_{\ell}(u) d u}{\left(s^{2}-u^{2}\right)^{\nu}} .
$$

Our particular condition is $[\mathcal{K}]_{\Delta_{-}}=\delta^{(d)}\left(x-x^{\prime}\right)$. The delta function can be expanded

$$
\delta^{(d)}\left(x-x^{\prime}\right)=\frac{\delta\left(r-r^{\prime}\right)}{r^{d-1}} \sum_{\ell} c_{\ell} Y_{\ell}\left(\hat{x}, \hat{x}^{\prime}\right)
$$

where

$$
c_{\ell}=\frac{1}{\mathcal{N}_{\ell}} \overline{Y_{\ell}\left(\hat{x}^{\prime}, \hat{x}^{\prime}\right)} \quad \text { with } \quad \mathcal{N}_{\ell}=\int_{S^{d-1}} d \Omega_{d-1}(\hat{x})\left|Y_{\ell}(\hat{x})\right|^{2} .
$$

This means that $\phi_{\ell}(r)=\frac{c_{\ell}}{r^{d-1}} \delta\left(r-r^{\prime}\right)$, and therefore

$$
g_{\ell}(s)=2 c_{\ell} \frac{\sin (\pi \nu)}{\pi} r^{\ell \ell} s^{\nu-\ell-d / 2+1} \frac{d}{d s}\left[\frac{\theta\left(s-r^{\prime}\right)}{\left(s^{2}-r^{\prime 2}\right)^{\nu}}\right] .
$$


Together with equations (B.2) and (B.6), this yields an explicit integral representation for the mixed bulk-boundary propagator $K_{+}$. The relevant integrals can be evaluated in terms of hypergeometric functions, and one can verify that (up to a change in conformal frame) the result matches (3.44).

\section{Spectral decomposition on $\boldsymbol{H}^{d}$}

Start with the metric (A.11) on $H^{d}$,

$$
d s^{2}=\frac{d w^{2}}{w(1+w)}+4 w(1+w) d \Omega_{d-1}^{2}(\gamma), \quad w \in[0, \infty)
$$

with $\gamma$ coordinates on $S^{d-1}$. We look for solutions to the equation

$$
-\nabla_{H^{d}}^{2} \Psi=\lambda \Psi
$$

Let $\ell$ index the harmonics $Y_{\ell}$ on the unit sphere $S^{d-1}$, and denote by $L_{\ell}$ the eigenvalues of $-\nabla_{S^{d-1}}^{2} ; L_{\ell}=k(k+d-2)$ for an integer $k$. Decomposing $\Psi=Y_{\ell} \psi(w)$, we have

$$
\left([w(1+w)]^{1-d / 2} \frac{d}{d w}[w(1+w)]^{d / 2} \frac{d}{d w}-[4 w(1+w)]^{-1} L_{\ell}+\lambda\right) \psi(w)=0 .
$$

This equation is hypergeometric, and has a unique solution that is finite as $w \rightarrow 0$ :

$$
\psi_{k, \sigma}(w)=\mathcal{N}_{k, \sigma} w^{k / 2}(1+w)^{1-(d+k) / 2}{ }_{2} F_{1}\left(\begin{array}{c}
\frac{1}{2}+i \sigma, \frac{1}{2}-i \sigma \\
\frac{d}{2}+k
\end{array} \mid-w\right)
$$

where we have expressed the eigenvalue in the form $\lambda=\sigma^{2}+\frac{(d-1)^{2}}{4}$.

We can find the normalized eigenfunctions in the following way. First of all, let $Y_{\ell}$ be normalized,

$$
\int_{S^{d-1}} d \Omega_{d-1}(\gamma) Y_{\ell}(\gamma) \overline{Y_{\ell^{\prime}}(\gamma)}=\delta_{\ell, \ell^{\prime}}
$$

The Olevskii transform gives a resolution of the radial delta function of the form

$$
\begin{aligned}
& \left(\frac{1+w}{w}\right)^{\frac{d}{2}+k-1} \delta\left(w-w^{\prime}\right)= \\
& \frac{1}{\pi} \int_{0}^{\infty} d \sigma\left|\frac{\Gamma\left(\frac{1}{2}+i \sigma\right) \Gamma\left(\frac{d-1}{2}+k+i \sigma\right)}{\Gamma(2 i \sigma) \Gamma\left(\frac{d}{2}+k\right)}\right|_{2}^{2} F_{1}\left(\begin{array}{c}
\frac{1}{2}+i \sigma, \frac{1}{2}-i \sigma \\
\frac{d}{2}+k
\end{array} \mid-w\right){ }_{2} F_{1}\left(\begin{array}{c}
\frac{1}{2}+i \sigma, \frac{1}{2}-i \sigma \\
\frac{d}{2}+k
\end{array} \mid-w^{\prime}\right)
\end{aligned}
$$

Setting

$$
\mathcal{N}_{k, \sigma}=\frac{2^{(1-d) / 2}}{\sqrt{\pi}} \frac{\Gamma\left(\frac{1}{2}+i \sigma\right) \Gamma\left(\frac{d-1}{2}+k+i \sigma\right)}{\Gamma(2 i \sigma) \Gamma\left(\frac{d}{2}+k\right)}
$$

and $\Psi_{\ell, \sigma}(w, \gamma)=Y_{\ell}(\gamma) \psi_{k, \sigma}(w)$, we obtain the identity

$$
\int_{0}^{\infty} d \sigma \sum_{\ell} \overline{\Psi_{\ell, \sigma}\left(w^{\prime}, \gamma^{\prime}\right)} \Psi_{\ell, \sigma}(w, \gamma)=\frac{1}{\sqrt{g_{H^{d}}}} \delta\left(w-w^{\prime}\right) \delta\left(\gamma-\gamma^{\prime}\right)=\frac{\sqrt{g_{S^{d-1}}}}{\sqrt{g_{H^{d}}}} \delta\left(w-w^{\prime}\right) \delta\left(\gamma, \gamma^{\prime}\right) .
$$


Similarly, from the inverse Olevskii transform we find that

$$
\int_{H^{d}} d^{d} x \sqrt{g_{H^{d}}} \Psi_{\ell, \sigma}(x) \overline{\Psi_{\ell^{\prime}, \sigma^{\prime}}(x)}=\delta_{\ell, \ell^{\prime}} \delta\left(\sigma-\sigma^{\prime}\right)
$$

where $x^{i}$ denote the coordinates on $H^{d}$.

Thus the functions $\Psi_{\ell, \sigma}$ form a complete basis for the normalizable functions on $H^{d}$.

$\mathbf{S O}(d, 1)$-invariant bifunctions. Our primary interest is in bifunctions on $H^{d}$, i.e. functions $u\left(x, x^{\prime}\right)$ of two points $x, x^{\prime} \in H^{d}$ that are symmetric and invariant under $\mathrm{SO}(d, 1)$, that are also eigenfunctions of the Laplacian. As with any function, it is possible to expand it with respect to eigenfunctions of the Laplacian $u_{\lambda}\left(x, x^{\prime}\right)$ satisfying the same properties. At fixed eigenvalue $\lambda_{\sigma}$, such functions can be decomposed as a sum over spherical harmonics of the $\Psi_{\ell, \sigma}$ functions:

$$
u_{\lambda_{\sigma}}\left(x, x^{\prime}\right)=\sum_{\ell} c_{\ell}\left(x^{\prime}\right) \Psi_{\ell, \sigma}(x) .
$$

Such a function depends only on the hyperbolic distance, and so it suffices to set $x^{\prime}=0$ (i.e. $w^{\prime}=0$ ). The expression is further rotationally invariant around $x^{\prime}=0$, which implies that only the $\ell=0$ mode contributes. We thus find

$$
u(x, 0)=c^{\prime} \Psi_{0, \sigma}(w) ;
$$

of course, for $\ell=0$ there is no dependence on the angular variables $\gamma$. To recover the general expression, we simply express the result in terms of the hyperbolic distance.

The invariant distance between the point $x^{\prime}$ with $w^{\prime}=0$ and the point $x=(w, \gamma)$ is

$$
d\left(x, x^{\prime}\right)=\int_{0}^{w}[s(1+s)]^{-1 / 2} d s=2 \sinh ^{-1}\left(w^{1 / 2}\right)
$$

which gives $4 w(1+w)=\sinh ^{2} d\left(x, x^{\prime}\right)$. A simple expression for the hyperbolic distance in Poincaré patch coordinates $\frac{d y^{2}+d \vec{x}^{2}}{y^{2}}$ can be given in terms of the cross-ratio $\chi_{d}^{2}$ :

$$
d\left(x, x^{\prime}\right)=\cosh ^{-1}\left(1+2 \chi_{d}^{2}\right), \quad \chi_{d}^{2}=\frac{\left(\vec{x}-\vec{x}^{\prime}\right)^{2}+\left(y-y^{\prime}\right)^{2}}{4 y y^{\prime}} .
$$

For $w^{\prime}=0, \chi_{d}^{2}=w$, so $u(x, 0)$ above can be covariantized to general $x^{\prime}$ by replacing $w \mapsto \chi_{d}^{2}$.

A bifunction of particular interest for us is

$$
J_{\sigma}\left(x, x^{\prime}\right)=\sum_{\ell} \Psi_{\ell, \sigma}(x) \overline{\Psi_{\ell, \sigma}\left(x^{\prime}\right)} .
$$

Equation (C.8) implies that $\int d \sigma J_{\sigma}\left(x, x^{\prime}\right)=\delta\left(x, x^{\prime}\right)$, which is invariant under $\operatorname{SO}(d, 1)$ transformations; because $\mathrm{SO}(d, 1)$ doesn't mix eigenvalues of the Laplacian, this implies that $J_{\sigma}\left(x, x^{\prime}\right)$ itself is invariant under $\mathrm{SO}(d, 1)$ transformations.

By acting with a conformal transformation, we can set $x^{\prime}=0$, in which case all modes but $k=0$ drop out. With $Y_{0}(\gamma)=\left(\operatorname{vol} S^{d-1}\right)^{-1 / 2}$,

$$
J_{\sigma}(x, 0)=\frac{1}{\operatorname{vol} S^{d-1}} \frac{2}{2^{d} \pi}(1+w)^{1-d / 2}\left|\frac{\Gamma\left(\frac{1}{2}+i \sigma\right) \Gamma\left(\frac{d-1}{2}+i \sigma\right)}{\Gamma(2 i \sigma) \Gamma\left(\frac{d}{2}\right)}\right|_{2}^{2} F_{1}\left(\begin{array}{c}
\frac{1}{2}+i \sigma, \frac{1}{2}-i \sigma \\
d / 2
\end{array} \mid-w\right) .
$$


As we saw above, we can find its value at general $x^{\prime}$ by replacing $w \mapsto \chi_{d}^{2}$ :

$$
\begin{aligned}
J_{\sigma}\left(x, x^{\prime}\right) & =\frac{1}{(4 \pi)^{d / 2}} \frac{\Gamma(d / 2)}{\pi}\left|\frac{\Gamma\left(\frac{1}{2}+i \sigma\right) \Gamma\left(\frac{d-1}{2}+i \sigma\right)}{\Gamma(2 i \sigma) \Gamma\left(\frac{d}{2}\right)}\right|^{2}\left(1+\chi_{d}^{2}\right)^{1-d / 2}{ }_{2} F_{1}\left(\begin{array}{c}
\frac{1}{2}+i \sigma, \frac{1}{2}-i \sigma \\
d / 2
\end{array} \mid-\chi_{d}^{2}\right) \\
& =\frac{1}{(4 \pi)^{d / 2}} \frac{\Gamma(d / 2)}{\pi}\left|\frac{\Gamma\left(\frac{1}{2}+i \sigma\right) \Gamma\left(\frac{d-1}{2}+i \sigma\right)}{\Gamma(2 i \sigma) \Gamma\left(\frac{d}{2}\right)}\right|^{2} F_{1}\left(\begin{array}{c}
\frac{d-1}{2}+i \sigma, \frac{d-1}{2}-i \sigma \\
d / 2
\end{array} \mid-\chi_{d}^{2}\right) . \quad(\text { C. } 17)
\end{aligned}
$$

We often require the value at coincidence:

$$
\mathcal{N}_{\sigma}:=J_{\sigma}(x, x)=\frac{1}{(4 \pi)^{d / 2}} \frac{\Gamma(d / 2)}{\pi}\left|\frac{\Gamma\left(\frac{1}{2}+i \sigma\right) \Gamma\left(\frac{d-1}{2}+i \sigma\right)}{\Gamma(2 i \sigma) \Gamma\left(\frac{d}{2}\right)}\right|^{2} .
$$

\section{Integral transforms}

In Janus coordinates, we make extensive use of a hypergeometric index integral transform. The transform in question is a generalization of the Mehler-Fock transform that was first discovered by Weyl [53]. His work was largely forgotten, and the same integral transform was later rediscovered by Titchmarsh [54] and Olevskii [55].

Let $a, c>0$, and $f(x)$ be a sufficiently well-behaved function (say, smooth and of compact support) on $\mathbb{R}_{+}$. The transform $\mathcal{J}_{a, c}\{f\}$ of $f$ is

$$
g(s)=\mathcal{J}_{a, c}\{f\}(s)=\int_{0}^{\infty} d x x^{a+c-1}(1+x)^{a-c}{ }_{2} F_{1}\left(\begin{array}{c}
a+i s, a-i s \\
a+c
\end{array} \mid-x\right) f(x) .
$$

The inversion theorem (see, e.g., [56]) states that $f(x)$ is recovered by the following formula:

$$
f(x)=\mathcal{J}_{a, c}^{-1}\{g\}(x)=\frac{1}{\pi} \int_{0}^{\infty} d s\left|\frac{\Gamma(a+i s) \Gamma(c+i s)}{\Gamma(2 i s) \Gamma(a+c)}\right|_{2}^{2} F_{1}\left(\begin{array}{c}
a+i s, a-i s \\
a+c
\end{array} \mid-x\right) g(s) .
$$

\section{E Hypergeometric functions}

The Gauss hypergeometric function is defined by

$$
{ }_{2} F_{1}\left(\begin{array}{c}
a, b \\
c
\end{array} \mid z\right)=\sum_{n=0}^{\infty} \frac{(a)_{n}(b)_{n}}{(c)_{n}} \frac{z^{n}}{n !}
$$

We also encounter the generalized hypergeometric function

$$
{ }_{p} F_{q}\left(\begin{array}{c}
a_{1}, \ldots, a_{p} \\
b_{1}, \ldots, b_{q}
\end{array} \mid z\right)=\sum_{n=0}^{\infty} \frac{\left(a_{1}\right)_{n} \cdots\left(a_{p}\right)_{n}}{\left(b_{1}\right)_{n} \cdots\left(b_{q}\right)_{n}} \frac{z^{n}}{n !} .
$$

Differential equation. Set $F(z)={ }_{2} F_{1}\left(\begin{array}{c}a, b \\ c\end{array} \mid z\right)$. Then $F$ satisfies

$$
z(1-z) F^{\prime \prime}+[c-(a+b+1) z] F^{\prime}-a b F=0 .
$$




\section{E.1 Identities}

\section{E.1.1 Euler identities}

$$
\begin{aligned}
{ }_{2} F_{1}\left(\begin{array}{c}
a, b \\
c
\end{array} \mid z\right) & =(1-z)^{c-a-b}{ }_{2} F_{1}\left(\begin{array}{c}
c-a, c-b \\
c
\end{array} \mid z\right) \\
& =(1-z)^{-a}{ }_{2} F_{1}\left(\begin{array}{c}
a, c-b \\
c
\end{array} \mid-\frac{z}{1-z}\right) \\
& =(1-z)^{-b}{ }_{2} F_{1}\left(\begin{array}{c}
c-a, b \\
c
\end{array} \mid-\frac{z}{1-z}\right)
\end{aligned}
$$

\section{E.1.2 Kummer's connection formulas}

Defining

$$
\begin{array}{rlrl}
\Phi_{1} & ={ }_{2} F_{1}\left(\begin{array}{c}
a, b \\
c
\end{array} \mid z\right) & \Phi_{2}=z^{1-c}{ }_{2} F_{1}\left(\begin{array}{c}
a-c+1, b-c+1 \\
2-c
\end{array} \mid z\right) \\
\Phi_{3}={ }_{2} F_{1}\left(\begin{array}{c}
a, b \\
a+b-c+1
\end{array} \mid 1-z\right) & \Phi_{4}=(1-z)^{c-a-b}{ }_{2} F_{1}\left(\begin{array}{c}
c-a, c-b \\
c-a-b+1
\end{array} \mid 1-z\right) \\
\Phi_{5}=(-z)^{-a}{ }_{2} F_{1}\left(\begin{array}{c}
a, a-c+1 \\
a-b+1
\end{array} \mid \frac{1}{z}\right) & \Phi_{6}=(-z)^{-b}{ }_{2} F_{1}\left(\begin{array}{c}
b-c+1, b \\
b-a+1
\end{array} \mid \frac{1}{z}\right)
\end{array}
$$

we have

$$
\begin{aligned}
\Phi_{1} & =\frac{\Gamma(c) \Gamma(c-a-b)}{\Gamma(c-a) \Gamma(c-b)} \Phi_{3}+\frac{\Gamma(c) \Gamma(a+b-c)}{\Gamma(a) \Gamma(b)} \Phi_{4} \\
& =\frac{\Gamma(c) \Gamma(b-a)}{\Gamma(b) \Gamma(c-a)} \Phi_{5}+\frac{\Gamma(c) \Gamma(a-b)}{\Gamma(a) \Gamma(c-b)} \Phi_{6} .
\end{aligned}
$$

These are guaranteed to be valid for $0<\Re z<1$ and $\Re z<0$, respectively; for general values of $z$ one needs to take care with the branch cuts.

\section{E.1.3 Sum relations}

The decomposition into conformal blocks of section 4.2 is accomplished using equation (4.3.11) of [57],

$$
{ }_{2} F_{1}\left(\begin{array}{c}
a, b \\
c
\end{array} \mid z\right)=\sum_{k=0}^{\infty} \frac{(\alpha)_{k}(\beta)_{k}}{k !(\gamma+k-1)_{k}}(-z)^{k}{ }_{4} F_{3}\left(\begin{array}{c}
a, b, \gamma+k-1,-k \\
\alpha, \beta, c
\end{array} \mid 1\right){ }_{2} F_{1}\left(\begin{array}{c}
\alpha+k, \beta+k \\
\gamma+2 k
\end{array} \mid z\right)
$$

valid for any choice of $\alpha, \beta, \gamma$ such that the identity makes sense. For our two-point function, ${ }_{4} F_{3}$ reduces to ${ }_{3} F_{2}$, and we apply Saalschütz's theorem

$$
{ }_{3} F_{2}\left(\begin{array}{c}
a, b,-k \\
c, a+b+1-c-k
\end{array} \mid 1\right)=\frac{(c-a)_{k}(c-b)_{k}}{(c)_{k}(c-a-b)_{k}} \quad k=0,1,2, \ldots
$$




\section{E.2 Integrals}

When the sum (E.2) converges uniformly on [0,1], Taylor expansion together with the beta integral implies

$$
\int_{0}^{1} d z z^{\mu-1}(1-z)^{\nu-1}{ }_{p} F_{q}\left(\begin{array}{c}
a_{1}, \ldots, a_{p} \\
b_{1}, \ldots, b_{q}
\end{array} \mid z\right)=\frac{\Gamma(\mu) \Gamma(\nu)}{\Gamma(\mu+\nu)} p+1 F_{q+1}\left(\begin{array}{c}
\mu, a_{1}, \ldots, a_{p} \\
\mu+\nu, b_{1}, \ldots, b_{q}
\end{array} \mid z\right)
$$

provided the integral exists. If the integrand is bounded as $z \rightarrow 1$ but diverges at $z=0$, subtraction of the power law divergences is equivalent to performing analytic continuation in $\mu$. Only a finite number of terms give rise to divergences; the most divergent contribution has the form $-\frac{z^{\mu}}{\mu}$.

\section{E.3 Index integrals of hypergeometric functions}

Let $a_{1}, a_{2}, a_{3}, a_{4} \in \mathbb{R}$, and consider the integral

$$
\frac{1}{\pi} \int_{0}^{\infty} d \sigma\left|\frac{\Gamma\left(a_{1}+i \sigma\right) \Gamma\left(a_{2}+i \sigma\right) \Gamma\left(a_{3}+i \sigma\right) \Gamma\left(a_{4}+i \sigma\right)}{\Gamma(2 i \sigma)}\right|_{p+2} F_{q}\left(\begin{array}{c}
a_{1}+i \sigma, a_{1}-i \sigma, b_{1}, \ldots, b_{p} \\
c_{1}, \ldots, c_{q}
\end{array} \mid z\right) .
$$

Expanding in powers of $z$ and using the De Branges-Wilson integral

$$
\frac{1}{\pi} \int_{0}^{\infty} d \sigma\left|\frac{\prod_{i=1}^{4} \Gamma\left(a_{i}+i \sigma\right)}{\Gamma(2 i \sigma)}\right|^{2}=\frac{\prod_{i<j} \Gamma\left(a_{i}+a_{j}\right)}{\Gamma\left(\sum_{i} a_{i}\right)}
$$

we see that it equals

$$
\frac{\prod_{i<j} \Gamma\left(a_{i}+a_{j}\right)}{\Gamma\left(\sum_{i} a_{i}\right)}{ }_{p+3} F_{q+1}\left(\begin{array}{c}
a_{1}+a_{2}, a_{1}+a_{3}, a_{1}+a_{4}, b_{1}, \ldots, b_{p} \\
\sum_{i} a_{i}, c_{1}, \ldots, c_{q}
\end{array} \mid z\right) .
$$

\section{E.4 Three-term relations for ${ }_{3} F_{2}(1)$}

The purpose of this section is to provide a sum rule for ${ }_{3} f_{2}\left(\begin{array}{c}a_{0}, a_{1}, a_{2} \\ b_{1}, b_{2}\end{array}\right)={ }_{3} F_{2}\left(\begin{array}{c}a_{0}, a_{1}, a_{2} \\ b_{1}, b_{2}\end{array} \mid 1\right)$. With $\mathbf{a}=\left(\begin{array}{ccc}a_{0} & a_{1} & a_{2} \\ & b_{1} & b_{2}\end{array}\right)$, define

$$
y(\mathbf{a})=\frac{\prod_{i=0}^{2} \sin \pi a_{i}}{\prod_{i=1}^{2} \sin \pi b_{i}} 3 f_{2}\left(\begin{array}{c}
a_{0}, a_{1}, a_{2} \\
b_{1}, b_{2}
\end{array}\right)
$$

and set

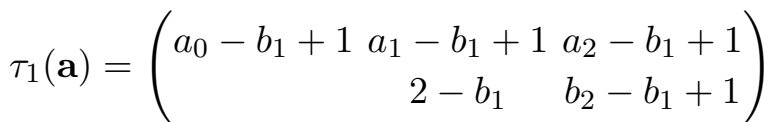

$$
\begin{aligned}
& \tau_{2}(\mathbf{a})=\left(\begin{array}{ccc}
a_{0}-b_{2}+1 & a_{1}-b_{2}+1 & a_{2}-b_{2}+1 \\
b_{1}-b_{2}+1 & 2-b_{2}
\end{array}\right) .
\end{aligned}
$$


We utilize the following standard 3-term relation (see, e.g., [58], whose notation we follow):

$$
y(\mathbf{a})+y\left(\tau_{1}(\mathbf{a})\right)+y\left(\tau_{2}(\mathbf{a})\right)=0 .
$$

Applying this to

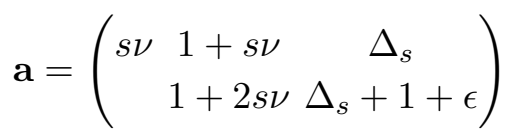

and taking the limit $\epsilon \rightarrow 0$ gives the relation

$$
-\frac{1}{2} \tan (\pi \nu) \sum_{s= \pm 1} s_{3} f_{2}\left(\begin{array}{c}
s \nu, 1+s \nu, \Delta_{s} \\
1+2 s \nu, \Delta_{s}+1
\end{array}\right)+\pi \frac{\Gamma\left(\Delta_{+}\right) \Gamma\left(\Delta_{-}\right)}{\Gamma\left(\frac{d}{2}\right) \Gamma\left(\frac{d}{2}+1\right)}=0 .
$$

\section{F Conventions for $\mathfrak{s u}(N)$}

Our conventions for $\mathfrak{s u}(N)$ and its affine algebras follow [47]. Here we collect some facts which are important for our section 6.2.

The dimension of $\mathfrak{s u}(N)$ is $N^{2}-1$, and the dual Coxeter number is $g^{\vee}=N$. Bases of generators are denoted $J^{a}, a=1, \ldots, N^{2}-1$. The weight and root lattice of $\mathfrak{s u}(N)$ can be realized in $\mathbb{R}^{N}$ with standard basis $e_{1}, \ldots e_{N}$ : the roots are given by $\alpha=e_{i}-e_{j}$ for $i \neq j$, and we define the positive roots to be those with $i<j$. A set of simple roots is then provided by $\alpha_{i}=e_{i}-e_{i+1}$ for $i=1, \ldots, N-1$. The root lattice consists of all vectors of the form $\sum_{i=1}^{N} n_{i} e_{i}$ with $n_{i} \in \mathbb{Z}$ and $\sum_{i} n_{i}=0$. The Weyl vector, given by half the sum of all positive roots, is represented by $\rho=\frac{1}{2} \sum_{i=1}^{N}(N+1-2 i) e_{i}$. The fundamental weights are $\omega_{i}=\sum_{j=1}^{i} e_{j}-\frac{i}{N} \sum_{j=1}^{N} e_{j}$ for $i=1, \ldots, N-1$; every weight is given by $\lambda=\sum_{i=1}^{N-1} \lambda_{i} \omega_{i}$ with Dynkin labels $\lambda_{i}$. In our case we need in particular the fundamental and antifundamental representations with highest weights $\omega_{1}$ and $\omega_{N-1}$, respectively. The fundamental representation contains the weights $\omega_{1}-\sum_{j=1}^{i} \alpha_{j}$, and the antifundamental representation contains the weights $\omega_{N-1}-\sum_{j=1}^{i} \alpha_{N-j}$ for $i=0,1, \ldots, N-1$ (empty sums are 0$)$. We also need the adjoint representation $\theta$, which has Dynkin labels $1,0, \ldots, 0,1$.

In the $\mathfrak{s u}(N)_{k}$ WZW model, the chiral fields $J^{a}(z)$ can be decomposed into modes $J_{n}^{a}, n \in \mathbb{Z}$, where $J_{0}^{a}$ act as $-J^{a}$ on the Virasoro highest weight states $|\lambda\rangle$ labeled by $\mathfrak{s u}(N)$ weights, and $J_{n}^{a}|\lambda\rangle=0$ for $n>0$. The $|\lambda\rangle$ have (chiral) conformal dimension $(\lambda, \lambda+2 \rho) / 2(k+N)$, where the inner product coincides with the standard one on $\mathbb{R}^{N}$. Highest weight operators with respect to the $\mathfrak{s u}(N)_{k}$ algebra only occur if $(\lambda, \theta) \leq k$.

The formula for the elements in the first column of the (symmetric) modular $S$ matrix is

$$
S_{\lambda 0}^{(k)}=\left|\operatorname{det}\left(\left(\alpha_{i}^{\vee}\right)_{j}\right)\right|^{-\frac{1}{2}}(k+N)^{-\frac{N-1}{2}} \prod_{\alpha>0} 2 \sin \left(\frac{\pi(\alpha, \lambda+\rho)}{k+N}\right) .
$$

In this formula, the $\alpha_{i}^{\vee}$ denote the coroots, which in $\mathbb{R}^{N}$ coincide with the roots.

For the coset $\mathfrak{s u}(N)_{k} \otimes \mathfrak{s u}(N)_{1} / \mathfrak{s u}(N)_{k+1}$, we recall that representations are given by a triple $\Lambda=\left(\lambda^{(k)}, \lambda^{(1)}, \lambda^{(k+1)}\right)$ of weights of the respective affine $\mathfrak{s u}(N)$ algebras. The condition that $\lambda^{(k)}+\lambda^{(1)}-\lambda^{(k+1)}$ needs to be in the root lattice then allows precisely one $\lambda^{(1)}$ for a given pair $\left(\lambda^{(k)}, \lambda^{(k+1)}\right)$. 
Open Access. This article is distributed under the terms of the Creative Commons Attribution License (CC-BY 4.0), which permits any use, distribution and reproduction in any medium, provided the original author(s) and source are credited.

\section{References}

[1] I. Brunner and D. Roggenkamp, Defects and bulk perturbations of boundary Landau-Ginzburg orbifolds, JHEP 04 (2008) 001 [arXiv:0712.0188] [INSPIRE].

[2] A. Konechny and C. Schmidt-Colinet, Entropy of conformal perturbation defects, J. Phys. A 47 (2014) 485401 [arXiv:1407.6444] [INSPIRE].

[3] I. Brunner and C. Schmidt-Colinet, Reflection and transmission of conformal perturbation defects, J. Phys. A 49 (2016) 195401 [arXiv:1508.04350] [INSPIRE].

[4] F. Gliozzi, P. Liendo, M. Meineri and A. Rago, Boundary and Interface CFTs from the Conformal Bootstrap, JHEP 05 (2015) 036 [arXiv: 1502.07217] [INSPIRE].

[5] D. Gaiotto, Domain Walls for Two-Dimensional Renormalization Group Flows, JHEP 12 (2012) 103 [arXiv:1201.0767] [INSPIRE].

[6] T. Dimofte, D. Gaiotto and R. van der Veen, $R G$ Domain Walls and Hybrid Triangulations, Adv. Theor. Math. Phys. 19 (2015) 137 [arXiv:1304.6721] [INSPIRE].

[7] D. Bak, M. Gutperle and S. Hirano, A dilatonic deformation of $A d S_{5}$ and its field theory dual, JHEP 05 (2003) 072 [hep-th/0304129] [INSPIRE].

[8] E. D'Hoker, J. Estes, M. Gutperle and D. Krym, Exact Half-BPS Flux Solutions in M-theory. I: Local Solutions, JHEP 08 (2008) 028 [arXiv:0806.0605] [INSPIRE].

[9] E. D'Hoker, J. Estes, M. Gutperle and D. Krym, Janus solutions in M-theory, JHEP 06 (2009) 018 [arXiv: 0904.3313] [INSPIRE].

[10] T. Nishioka and H. Tanaka, Lifshitz-like Janus Solutions, JHEP 02 (2011) 023 [arXiv:1010.6075] [INSPIRE].

[11] D. Bak, M. Gutperle and R.A. Janik, Janus Black Holes, JHEP 10 (2011) 056 [arXiv: 1109.2736] [INSPIRE].

[12] M. Chiodaroli, J. Estes and Y. Korovin, Holographic two-point functions for Janus interfaces in the D1/D5 CFT, JHEP 04 (2017) 145 [arXiv:1612.08916] [INSPIRE].

[13] N. Bobev, K. Pilch and N.P. Warner, Supersymmetric Janus Solutions in Four Dimensions, JHEP 06 (2014) 058 [arXiv: 1311.4883] [INSPIRE].

[14] P. Karndumri and K. Upathambhakul, Supersymmetric RG flows and Janus from type-II orbifold compactification, Eur. Phys. J. C 77 (2017) 455 [arXiv:1704.00538] [INSPIRE].

[15] I.R. Klebanov and E. Witten, AdS/CFT correspondence and symmetry breaking, Nucl. Phys. B 556 (1999) 89 [hep-th/9905104] [INSPIRE].

[16] M.R. Gaberdiel and R. Gopakumar, An AdS $S_{3}$ Dual for Minimal Model CFTs, Phys. Rev. D 83 (2011) 066007 [arXiv:1011.2986] [INSPIRE].

[17] I.N. Sneddon, Mixed Boundary Value Problems in Potential Theory, North Holland, (1966).

[18] O. Aharony, O. DeWolfe, D.Z. Freedman and A. Karch, Defect conformal field theory and locally localized gravity, JHEP 07 (2003) 030 [hep-th/0303249] [INSPIRE]. 
[19] M. Billò, V. Gonçalves, E. Lauria and M. Meineri, Defects in conformal field theory, JHEP 04 (2016) 091 [arXiv: 1601.02883] [INSPIRE].

[20] R. Rattazzi, V.S. Rychkov, E. Tonni and A. Vichi, Bounding scalar operator dimensions in 4D CFT, JHEP 12 (2008) 031 [arXiv:0807.0004] [INSPIRE].

[21] D.M. McAvity and H. Osborn, Conformal field theories near a boundary in general dimensions, Nucl. Phys. B 455 (1995) 522 [cond-mat/9505127] [INSPIRE].

[22] I. Affleck and A.W.W. Ludwig, Universal noninteger 'ground state degeneracy' in critical quantum systems, Phys. Rev. Lett. 67 (1991) 161 [INSPIRE].

[23] D. Bak, A. Gustavsson and S.-J. Rey, Conformal Janus on Euclidean Sphere, JHEP 12 (2016) 025 [arXiv: 1605.00857] [InSPIRE].

[24] D.E. Diaz and H. Dorn, Partition functions and double-trace deformations in AdS/CFT, JHEP 05 (2007) 046 [hep-th/0702163] [INSPIRE].

[25] C.P. Herzog, K.-W. Huang and K. Jensen, Universal Entanglement and Boundary Geometry in Conformal Field Theory, JHEP 01 (2016) 162 [arXiv:1510.00021] [INSPIRE].

[26] D. Fursaev, Conformal anomalies of CFT's with boundaries, JHEP 12 (2015) 112 [arXiv: 1510.01427] [INSPIRE].

[27] S.N. Solodukhin, Boundary terms of conformal anomaly, Phys. Lett. B 752 (2016) 131 [arXiv: 1510.04566] [INSPIRE].

[28] S. El-Showk and K. Papadodimas, Emergent Spacetime and Holographic CFTs, JHEP 10 (2012) 106 [arXiv:1101.4163] [INSPIRE].

[29] R. Jost, The General Theory of Quantized Fields, AMS, Providence, RI, U.S.A. (1965).

[30] M.R. Gaberdiel and R. Gopakumar, Minimal Model Holography, J. Phys. A 46 (2013) 214002 [arXiv: 1207.6697 ] [INSPIRE].

[31] C. Ahn, The Large-N 't Hooft Limit of Coset Minimal Models, JHEP 10 (2011) 125 [arXiv:1106.0351] [INSPIRE].

[32] C. Ahn, The Large-N 't Hooft Limit of Kazama-Suzuki Model, JHEP 08 (2012) 047 [arXiv: 1206.0054] [INSPIRE].

[33] D. Roggenkamp and K. Wendland, Limits and degenerations of unitary conformal field theories, Commun. Math. Phys. 251 (2004) 589 [hep-th/0308143] [INSPIRE].

[34] I. Runkel and G.M.T. Watts, A nonrational CFT with $c=1$ as a limit of minimal models, JHEP 09 (2001) 006 [hep-th/0107118] [INSPIRE].

[35] M.R. Gaberdiel and P. Suchanek, Limits of Minimal Models and Continuous Orbifolds, JHEP 03 (2012) 104 [arXiv: 1112.1708] [INSPIRE].

[36] S. Fredenhagen and C. Restuccia, The large level limit of Kazama-Suzuki models, JHEP 04 (2015) 015 [arXiv: 1408.0416] [INSPIRE].

[37] M.R. Gaberdiel and R. Gopakumar, Higher Spins 83 Strings, JHEP 11 (2014) 044 [arXiv: 1406.6103] [INSPIRE].

[38] D. Altschuler, M. Bauer and H. Saleur, Level rank duality in nonunitary coset theories, J. Phys. A 23 (1990) L789 [inSPIRE].

[39] C. Ahn, D. Bernard and A. LeClair, Fractional Supersymmetries in Perturbed Coset CFTs and Integrable Soliton Theory, Nucl. Phys. B 346 (1990) 409 [INSPIRE]. 
[40] S. Giombi and I.R. Klebanov, One Loop Tests of Higher Spin AdS/CFT, JHEP 12 (2013) 068 [arXiv: 1308.2337] [INSPIRE].

[41] C. Crnkovic, R. Paunov, G.M. Sotkov and M. Stanishkov, Fusions of Conformal Models, Nucl. Phys. B 336 (1990) 637 [inSPIRE].

[42] A. Recknagel, Permutation branes, JHEP 04 (2003) 041 [hep-th/0208119] [INSPIRE].

[43] H. Ishikawa, Boundary states in coset conformal field theories, Nucl. Phys. B 629 (2002) 209 [hep-th/0111230] [INSPIRE].

[44] T. Quella and V. Schomerus, Symmetry breaking boundary states and defect lines, JHEP 06 (2002) 028 [hep-th/0203161] [INSPIRE].

[45] T. Quella, I. Runkel and G.M.T. Watts, Reflection and transmission for conformal defects, JHEP 04 (2007) 095 [hep-th/0611296] [INSPIRE].

[46] P. Bouwknegt and K. Schoutens, W symmetry in conformal field theory, Phys. Rept. 223 (1993) 183 [hep-th/9210010] [INSPIRE].

[47] P. Di Francesco, P. Mathieu and D. Senechal, Conformal Field Theory, Springer New York (1997), [https://doi.org/10.1007/978-1-4612-2256-9].

[48] V.S. Dotsenko and V.A. Fateev, Conformal Algebra and Multipoint Correlation Functions in Two-Dimensional Statistical Models, Nucl. Phys. B 240 (1984) 312 [INSPIRE].

[49] C.-M. Chang and X. Yin, Correlators in $W_{N}$ Minimal Model Revisited, JHEP 10 (2012) 050 [arXiv:1112.5459] [INSPIRE].

[50] P. Liendo, L. Rastelli and B.C. van Rees, The Bootstrap Program for Boundary CFT $T_{d}$, JHEP 07 (2013) 113 [arXiv: 1210.4258] [InSPIRE].

[51] R. Gopakumar, A. Kaviraj, K. Sen and A. Sinha, A Mellin space approach to the conformal bootstrap, JHEP 05 (2017) 027 [arXiv:1611.08407] [INSPIRE].

[52] M. Abramowitz and I.A. Stegun, Handbook of Mathematical Functions with Formulas, Graphs, and Mathematical tables, Wiley-Interscience, New York U.S.A., (1972).

[53] H. Weyl, Über gewönliche lineare Differentialgleichungen mit singulären Stellen und ihre Eigenfunktionen, Göttinger Nachrichten (1910) 442-467, reprinted in H. Weyl, Gessamelte Abhandlungen I, Springer, (1968), pp. 222-247.

[54] E.C. Titchmarsh, Eigenfunction expansions with second-order differential operators, Oxford, Clarendon Press, (1946).

[55] M.N. Olevskii, On the representation of an arbitrary function in the form of an integral with a kernel containing a hypergeometric function (in Russian), Doklady Akad. Nauk SSSR (N.S.) 69 (1949) 11.

[56] Y.A. Neretin, Index hypergeometric transform and imitation of analysis of Berezin kernels on hyperbolic spaces, Sbornik Math. 192 (2001) 403 [math/0104035].

[57] A. Erdélyi ed., Higher Transcendental Functions, Vol. 1, McGraw-Hill, New York U.S.A., (1953).

[58] A. Ebisu and K. Iwasaki, Three-Term Relations for 3F2(1), arXiv:1604.00480. 